(C) 1999 International Press

Adv. Theor. Math. Phys. 3 (1999) 1-81

\title{
Non-Spherical Horizons, I
}

\author{
David R. Morrison and M. Ronen Plesser \\ Center for Geometry and Theoretical Physics \\ Box 90318, Duke University \\ Durham, NC 27708-0318
}

\begin{abstract}
We formulate an extension of Maldacena's AdS/CFT conjectures to the case of branes located at singular points in the ambient transverse space. For singularities which occur at finite distance in the moduli space of $\mathrm{M}$ or $F$ theory models with spacetime-filling branes, the conjectures identify the worldvolume theory on the $p$-branes with a compactification of $\mathrm{M}$ or IIB theory on $\mathrm{AdS}_{p+2} \times H^{D-p-2}$. We show how the singularity determines the horizon $H$, and demonstrate the relationship between global symmetries on the worldvolume and gauge symmetries in the $A d S$ model. As a first application, we study some singularities relevant to the D3-branes required in four-dimensional $F$ theory. For these we are able to explicitly derive the low-energy field theory on the worldvolume and compare its properties to predictions from the dual $A d S$ model. In particular, we examine the baryon spectra of the models and the fate of the Abelian factors in the gauge group.
\end{abstract}

e-print archive: http://xxx.lanl.gov/abs/hep-th/9810201

*On leave from Department of Particle Physics, Weizmann Institute of Science, Rehovot, Israel 
Spacetime-filling branes have emerged as an essential feature of string and M-theory compactifications in at least three contexts: (1) new branches of the heterotic string in six dimensions with "extra" tensor multiplets, which can be represented by a Hořava-Witten-type compactification of M-theory on $\left(S^{1} / \mathbb{Z}_{2}\right) \times K 3$ but with extra spacetime-filling M5-branes representing the extra tensor multiplets $[1,2,3]$; (2) $F$-theory models in four dimensions (which can be regarded as compactifications of the IIB string with D7-branes included) which in general require spacetime-filling D3-branes to cancel a tadpole anomaly [4]; and (3) M-theory models in three dimensions, which require spacetime-filling M2-branes to cancel a similar tadpole anomaly [4]. In each of these cases, the spacetime-filling brane meets the compactifying space at a single point, and the string or M-theory remains finite near the brane. $^{1}$

Remarkably, this short list of branes (M5, D3, and M2) is precisely the list of branes for which a certain scaling limit is expected to lead to a "boundary" conformal field theory in the recent $A d S / C F T$ conjectures $[5,6,7]$. In fact, the scaling limit can be taken even when the space transverse to the branes is curved, as in the compactification scenarios above. The details of the metric far from the location $y_{0}$ of the brane in the transverse space become irrelevant; for the purposes of studying the scaling limit, the metric on the compactifying space can be approximated by some metric on its tangent space $T_{y_{0}}$ at $y_{0}$. In the scaling limit, the rescaled supergravity metric approaches a metric of the form $A d S_{p+2} \times S^{k}$ in which the anti-de Sitter space has been formed out of the worldvolume of the brane and the radial direction within $T_{y_{0}}$, and $S^{k}$ is the unit sphere within $T_{y_{0}}$.

Maldacena's conjecture proposes that the $\mathrm{M}$ or string theory on this space $A d S_{p+2} \times S^{k}$, with $N$ units of flux of the supergravity $k$-form field strength through $S^{k}$, is dual to a specific conformal field theory on the boundary of $A d S_{p+2}$. The conjecture applies to the large $N$ limit when a large number of these branes have been brought together; in the compactification context, fairly large values of $N$ can be obtained by bringing together all available branes in a given model.

Virtually all points ${ }^{2}$ in the compactifying space have identical behavior in this scaling limit. That situation changes, however, if we consider a compactifying space which itself has singularities. Such spaces are necessary additions if one wishes to study the complete moduli space of string compactifications, since singularities can be acquired at special values of

\footnotetext{
${ }^{1}$ This last requirement excludes consideration of $F$-theory models in eight dimensions with the D7-brane being spacetime-filling.

${ }^{2}$ The exception is the four-dimensional $F$-theory models, where points located along the D7-branes behave differently; in particular, the string coupling becomes infinite at such points. The behavior of D3-branes at such points has recently been determined $[8,9,10]$, and we will not consider them here.
} 
moduli (occurring at finite distance in the moduli space). We have learned much interesting physics in the past few years by studying what happens at such singularities. Each of the three compactification scenarios above allows for singularities to be acquired, and once the space is singular, there is no apparent obstruction to moving the branes to the singular point.

What should we expect to happen if we locate $N$ branes at such a singular point? The first step is to understand the geometry of the scaling limit. Instead of comparing the rescaled metric to a metric on the tangent space $T_{y_{0}}$, when $y_{0}$ is a singular point we should compare to a metric on the tangent cone, which is topologically the product of $\mathbb{R}^{+}$and a $k$-dimensional "horizon" $H^{k}$. The key difference between smooth and singular points is that the horizon is no longer a sphere. We propose that the standard behavior one should expect for branes at a singularity in the scaling limit is a model of the form $A d S_{p+2} \times H^{k}$, with the horizon $H$ consisting of all points at some fixed distance from $y_{0}$. Moreover, the theory on $A d S_{p+2} \times H^{k}$ should have at least as much unbroken supersymmetry as the original compactified $M$ or string theory.

Under these conditions, the generalization of Maldacena's conjecture is clear: the $M$ or IIB theory compactified on $A d S_{p+2} \times H^{k}$ with $N$ units of $k$-flux through $H^{k}$ should be dual to a conformal field theory $\mathcal{C}_{N}$ (depending on the singularity), with a large $N$ 't Hooft-type limit being a field theory limit on the stringy side. In order for this conjecture to be meaningful, however, we need to find ways of identifying the families of conformal field theories $\mathcal{C}_{N}$. Note that the $\mathrm{M}$ or string moduli (including the locations of the branes) should determine the parameters of the conformal field theory. Since we have good geometric control of those moduli at weak coupling, we can expect good geometric interpretations of the field theory parameters. We exploit this idea systematically throughout this paper, using geometry to aid in our determination and investigation of the corresponding field theories.

The plan of the paper is as follows. In section 1, we review the scaling limit in detail and explain how it must be modified in the present context. We also discuss some generic features of the singularities we will study.

In section 2, we find a geometric characterization of the amount of supersymmetry carried by the limiting conformal field theories. Using existing mathematical theorems, we show that the $R$-symmetry group of the conformal field theory acts by isometries on the corresponding horizon manifold $H$. This discussion is closely related to the differential geometry of $H$.

In section 3 , we present a very brief discussion of these theories for M2branes and M5-branes. In the case of M2-branes, we merely give a few examples; in the case of M5-branes, we sketch the answer, deferring details to a companion publication [11]. 
In section 4 , we give a detailed analysis of the singularities occurring in D3-brane theories, focusing on a few particular classes which exhibit many of the general features. The tool here is algebraic geometry, and we find a nice correspondence with some of the results obtained earlier via differential geometry.

In section 5 , we study a particular singularity-a $\mathbb{Z}_{2} \times \mathbb{Z}_{2}$ orbifold-in considerable detail. This theory has been studied before in the literature, but our focus is on giving more detail and in particular in extracting geometric interpretations for field theory parameters and moduli. This is then applied in section 6 , where we present a number of new examples, all derived in some way from the $\mathbb{Z}_{2} \times \mathbb{Z}_{2}$ orbifold.

In section 7 we discuss our conclusions.

Extensions of the $A d S / C F T$ correspondence to supersymmetric theories with less than maximal supersymmetry have been extensively studied in the past few months, either by orbifold constructions [12]-[23] by reduction on Hopf fibrations [19], by "brane box" constructions [24, 25], or by compactification on homogeneous spaces [26] or other Einstein manifolds [27]. In fact the general idea of interpreting branes with less than maximal supersymmetry in terms of compactifications on spaces of the form $A d S \times H$ for interesting manifolds $H$ goes back at least as far as [28].

Particularly important to the development of our approach were early versions of it which appeared in [29] and [9]. While this work was in progress, we became aware of several overlapping projects: Klebanov and Witten [30] independently studied the field theories for D3-branes at a conifold singularity - one of the examples we discuss in section 6-and Gubser [31] and Gubser and Klebanov [32] studied some further aspects of those theories, overlapping our results to a certain extent. In addition, Acharya, Figueroa-O'Farrill, Hull, and Spence [33, 34] have independently considered the relationship between supersymmetry and the geometry of the horizon manifold; there is substantial overlap between their work and section 2 of this paper.

A preliminary version of the present work was discussed by the first author in a talk at the Strings '98 conference in Santa Barbara.

\section{The scaling limit}

We've learned, in studying $\mathrm{M}$ and string theory compactifications, of several interesting examples in which part of the background necessarily involves some spacetime-filling branes. We will focus in this paper on three of these, 
involving M2-branes, D3-branes, and M5-branes.

The first example is M-theory models in $2+1$ dimensions obtained by compactifying on some eight-manifold with holonomy group contained in Spin(7). As shown by Sethi, Vafa and Witten, there is a tadpole cancellation requirement of an insertion of $\chi / 24$ M2-branes, where $\chi$ is the Euler characteristic of the eight-manifold [4].

Second, consider an $F$-theory model in $3+1$ dimensions, constructed out of an elliptically fibered Calabi-Yau fourfold. We will regard this as a IIB string theory compactification on the base of the elliptic fibration (which is a six-manifold), with D7-branes wrapped around the four-manifolds along which the fibers in the elliptic fibration have degenerated. A similar tadpole cancellation requirement insists that we include $\chi / 24$ spacetime-filling D3branes in the background [4], where $\chi$ is the Euler characteristic of the Calabi-Yau fourfold.

A third example of this nature is found in the Hořava-Witten description [35] of the $E_{8} \times E_{8}$ string compactified on K3, thought of now as M-theory compactified on $\left(S^{1} / \mathbb{Z}_{2}\right) \times K 3$ with some $E_{8} \times E_{8}$ gauge fields on the HoravaWitten 9-branes (HW9-branes). In models where some M5-branes associated to small instantons are allowed to move off of the HW9-branes (becoming spacetime-filling M5-branes), interesting new branches of the theory arise [1]-[3]. These new branches can be studied from a dual perspective using $F$-theory, which is one of the reasons that we know that they exist $[3,36,37]$. There is a limit of $24 \mathrm{M} 5$-branes which can play a role in these theories, since 24 is the maximum number of small instantons which can occur here.

The limitation on the total number of branes in these compactification scenarios is an artifact of global features of the compactification space which are irrelevant in the appropriate scaling limit. In fact, there is no local reason to limit the number of branes, and one can study local versions of these compactification models with arbitrarily large numbers of spacetime-filling branes. (This is analogous to using ALE spaces in place of K3 surfaces to study certain local aspects of compactification, and to various other similar "geometric engineering" tricks.) We will do so henceforth whenever it is convenient for our analysis.

When we allow the compactifying space to acquire singularities, the requirement that the limiting value of moduli be at finite distance is extremely restrictive on the singularity types which can occur. When the compactifying space can be described by means of algebraic geometry (which further restricts the holonomy in $2+1$ dimensional models to being contained within $S U(4))$, the only singularities which can appear [38, 39] are those known as Gorenstein canonical singularities. Briefly put, these are singularities in complex dimension $n$ for which there exists a non-vanishing holomorphic 
$n$-form near the singularity which on any smooth blowup of the space extends to a holomorphic form. Although not all of these singularities can be embedded in Calabi-Yau manifolds, we should still expect to be able to use any space with such a singularity to "compactify" our M or string theory models.

Returning to the case of smooth compactifying manifolds, the low-energy theory in all three of our compactification scenarios has a supergravity description, in which the spacetime-filling branes are included by means of a "warped product" metric. Consider a flat $p$-brane with worldvolume $M^{p+1}$ $((p+1)$-dimensional Minkowski space) in a spacetime of dimension $D$, in which the transverse space $I^{k+1}$ need not be flat. The values of $(p, D)$ of interest here are $(2,11),(3,10)$, and $(5,11)$, and the dimension of the internal space $k+1=D-p-1$ takes the values 8,6 and 5 . The most general form of a metric which preserves $(p+1)$-dimensional Poincaré invariance is

$$
d s^{2}=\phi(y) d x_{M}^{2}+\widetilde{d y}_{I}^{2},
$$

where $\phi(y)$ is a positive function on $I$ called the warping factor. We use the notation $M \times{ }_{w} I$ to denote such a warped product. We treat $x$ and $y$ as dimensionful coordinates on $M$ and $I$, but note that the warping factor $\phi(y)$ is dimensionless and so $y$ must be measured with respect to a specific length scale in order to define $\phi$. We will use the $D$-dimensional Planck length $\ell=\ell_{D}$ so that $\phi$ is actually a function of $y / \ell$.

To get a solution to the supergravity equations of motion, we follow $[40,29,9]^{3}$ and use a special form of eq. (1.1) in which the warping factor has been written as a power of another function, and the metric on $I$ has been rewritten with a conformal factor which is a different power of the warping factor:

$$
d s^{2}=f(y)^{(3-k) /(k-1)} d x_{M}^{2}+f(y)^{2 /(k-1)} d y_{I}^{2} .
$$

This metric must be accompanied by an expectation value for the supergravity $k$-form field strength

$$
\left[d\left(f(y)^{-1}\right) \wedge d \operatorname{vol}_{M}+\star\left(d\left(f(y)^{-1}\right) \wedge d \operatorname{vol}_{M}\right)\right]_{k}
$$

where $[\cdots]_{k}$ denotes the projection onto the $k$-form piece. The equation of motion for $f(y)$ demands that it be a harmonic function on $I$ away from the location of the branes. (In the case of the M2-brane, there is a more general form of this solution in which the $k$-form field strength also has a component in the internal space, and the equation of motion for the warping factor is suitably modified [40].)

\footnotetext{
${ }^{3}$ The metric in [40] is written in terms of $\Delta(y)=f(y)^{\frac{2}{3}}$.
} 
The original supergravity solutions for the D3-brane [41], the M2-brane [42], and the M5-brane [43] took exactly this form (1.2), using the specific function

$$
f(y)=1+\frac{c_{k} g^{11-D} N}{(r / \ell)^{k-1}}
$$

which depends only on the distance $r$ from the brane; there must also be a source term in the equation of motion for the function $f(y)$, which satisfies:

$$
\Delta f=\tilde{c}_{k} g^{11-D} N \delta\left(y-y_{0}\right) .
$$

In these formulas, $N$ denotes the number of branes which are located at $y_{0}$, the (dimensionful) constant $\tilde{c}_{k}$ is determined by flux quantization rules for the particular type of branes being studied, and $-\tilde{c_{k}} /(k-1) c_{k}$ is the volume of a unit sphere of dimension $k$. The string coupling $g$ only enters when $D=10$.

Incorporating branes into more general solutions of the form (1.2) is then straightforward: if we have $N_{j}$ branes located at $y_{j} \in I$ then the warping factor should satisfy

$$
\Delta f=\tilde{c}_{k} g^{11-D} \sum_{j} N_{j} \delta\left(y-y_{j}\right) .
$$

When we include branes into more general compactifications, we need not demand that the metric be asymptotically flat, so our solutions to eq. (1.6) will be more general than eq.(1.4).

In order for a supergravity solution on the warped product $M^{p+1} \times{ }_{w} I$ to retain some supersymmetry in the effective $(p+1)$-dimensional theory, there must be some appropriate spinors defined on $M \times_{w} I$. The component of such a spinor lying in the internal space $I$ must be covariantly constant with respect to the metric $d y_{I}^{2}$. (This was the primary reason for including an appropriate power of the warping factor multiplying $d y_{I}^{2}$ in eqn. (1.2).) If there is an $\mathcal{N}$-dimensional space of covariantly constant spinors, then the resulting theory has $\mathcal{N}$ supersymmetries. In particular, whenever $\mathcal{N}>0$, the metric $d y_{I}^{2}$ must be Ricci-flat, and must therefore have a holonomy group which is a proper subgroup of $S O(k+2)$.

Consider $N$ branes at $y_{0}$, measuring $r$ from $y_{0}$. We locally rewrite the metric on $I$ in the form

$$
d y_{I}^{2}=r^{2}\left(\frac{d r^{2}}{r^{2}}+d \Omega_{H}^{2}+O\left(\frac{r}{\ell}\right)\right)
$$

where $d \Omega_{H}^{2}$ is a dimensionless metric on the "horizon" manifold $H^{k}$ - the unit sphere. Since $f$ satisfies eqn.(1.5), it has precisely the same local singularity as eqn.(1.4), i.e., there is some regular function $f_{0}(y)$ with

$$
f(y)=\frac{c_{k} g^{11-D} N}{(r / \ell)^{k-1}}+f_{0}(y)=\frac{c_{k} g^{11-D} N}{(r / \ell)^{k-1}}\left(1+O\left(\frac{r}{\ell}\right)\right) .
$$


It follows that the conformal factor in the metric on $I$ can be written in the form

$$
f(y)^{2 /(k-1)}=\frac{\ell^{2}}{r^{2}}\left(c_{k} g^{11-D} N\right)^{2 /(k-1)}\left(1+O\left(\frac{r}{\ell}\right)\right) .
$$

We now, following [5], introduce a new variable $u$ satisfying

$$
\frac{r}{\ell}=(u \ell)^{2 /(k-3)}
$$

and consider a scaling limit with $u$ held fixed and $\ell \rightarrow 0$. We are interested in the behavior of the dimensionless two-tensor $d s^{2} / \ell^{2}$ (the metric in Planck units) in this scaling limit. We write

$$
\frac{d s^{2}}{\ell^{2}}=\frac{1}{\ell^{2}} f(y)^{(3-k) /(k-1)} d x_{M}^{2}+\frac{r^{2}}{\ell^{2}} f(y)^{2 /(k-1)}\left(\frac{d r^{2}}{r^{2}}+d \Omega_{H}^{2}+O\left(\frac{r}{\ell}\right)\right)
$$

and then change variables (using also eqns. (1.8) and (1.9)) to get

$$
\begin{aligned}
\frac{d s^{2}}{\ell^{2}}= & \left(c_{k} g^{11-D} N\right)^{(3-k) /(k-1)} u^{2} d x_{M}^{2}+\left(c_{k} g^{11-D} N\right)^{2 /(k-1)} \\
& \times\left(\left(\frac{2}{k-3}\right)^{2} \frac{d u^{2}}{u^{2}}+d \Omega_{H}^{2}\right)+O(u \ell)
\end{aligned}
$$

so that in the limit, $d s^{2} / \ell^{2}$ approaches

$$
\begin{aligned}
& \left(c_{k} g^{11-D} N\right)^{(3-k) /(k-1)} u^{2} d x_{M}^{2}+\frac{4\left(c_{k} g^{11-D} N\right)^{2 /(k-1)}}{(k-3)^{2}} \frac{d u^{2}}{u^{2}} \\
& +\left(c_{k} g^{11-D} N\right)^{2 /(k-1)} d \Omega_{H}^{2} .
\end{aligned}
$$

This limiting metric (1.13) has two interpretations: on the one hand, it is a warped product of Minkowski space and the cone $C(H)$ over the horizon $H$, where the cone metric is defined to be

$$
d y_{C(H)}^{2}=d u^{2}+u^{2} d \Omega_{H}^{2}
$$

and the warped product structure is given by

$$
u^{2} d x_{M}^{2}+u^{-2} d y_{C(H)}^{2}
$$

(suppressing constants for clarity). On the other hand, (1.13) can be interpreted as a product metric on $A d S_{p+2} \times H^{k}$. The cone $C(H)$ with its limiting metric should be considered as a kind of linearization of the original Ricci-flat metric near $y_{0}$, the location of the brane.

We now consider what happens if the original internal space $I$ had singularities, and we are attempting to place $N$ branes at a singular point $y_{0}$. As discussed in the introduction, we propose that the standard behavior one 
should expect in this situation is that there is a horizon $H^{k}$ (points at "unit distance" from $y_{0}$ ) whose cone $C(H)$ provides a good linearization of the limiting Ricci-flat metric near $y_{0}$. More precisely, we will assume that $H$ has a (dimensionless) "metric" $d \Omega_{H}^{2}$ such that near $y_{0}$,

$$
d y_{I}^{2}=r^{2}\left(\frac{d r^{2}}{r^{2}}+d \Omega_{H}^{2}+O\left(\frac{r}{\ell}\right)\right)
$$

for some radial coordinate $r$ centered at $y_{0}$.

When (1.16) is satisfied, the analysis above can be repeated verbatim: nowhere did we assume that $H$ is a sphere. The conclusion is that the scaling limit of $d s^{2} / \ell^{2}$ can be interpreted as either a warped product $M^{p+1} \times{ }_{w} C(H)$, or as a direct product $A d S_{p+2} \times H$. In particular, for any Gorenstein canonical singularity for which (1.16) holds, there must exist an Einstein metric on $H$ whose corresponding cone $C(H)$ serves as the leading approximation to limiting Ricci-flat metrics from nearby smooth compactifications.

We can thus formulate a general program as follows. For each type of Gorenstein canonical singularity satisfying (1.16), we should first carefully study the horizon $H$, determining both its topology and the set of Einstein metrics on it whose cones could arise as limits from Ricci-flat metrics. For each such metric, we expect that $M$ or IIB theory compactified on $A d S_{p+2} \times$ $H$ with $N$ units of flux through $H$ will determine a conformal field theory on the boundary of $A d S_{p+2}$. Ultimately, we would like to find more direct descriptions of these conformal field theories.

There is an important subtlety which arises when the singularity at $y_{0}$ is not isolated: the horizon will intersect the singular locus and will not itself be smooth. Our program should be modified in such cases to allow for singularities on $H$, and to search for Einstein "metrics" with a specified type of singular behavior. In the case of M5-brane and D3-brane theories, the singularity of the horizon is an ADE singularity (in real codimension four), and it is natural to expect an orbifold-type "metric" at the singularity. For M2-branes, the horizon can have a Gorenstein canonical threefold singularity in real codimension six and it is not clear what kind of singular behavior of the metric to expect there.

An important aspect of our general program is determining how much unbroken supersymmetry each of these models have; it is to this issue that we turn next.

\footnotetext{
${ }^{4}$ As noted below, in certain circumstances $H$ has singularities and we will need a generalized form of metric.
} 


\section{Supersymmetry and holonomy}

In the scaling limit, we found a compactification on a space of the form $A d S \times H$. To have a supergravity solution, we need an Einstein metric on $H$. Moreover, if the effective theory on $A d S$ is to retain some supersymmetry, we need appropriate spinors defined on $A d S \times H$. The condition on the $H$ component of the spinor is that it should be a so-called Killing spinor, so the dimension of the space of Killing spinors counts the number of supersymmetries of the associated theory.

Metrics for which Killing spinors exist have been extensively studied in the mathematics literature. The key result for our purposes is a theorem of Bär [44], which establishes a natural isomorphism between the space of Killing spinors on $H$ and the space of covariantly constant spinors on the cone $C(H)$. This is exactly what should be expected if our basic scenario is valid: the limit of the Ricci-flat metric on the original internal space $I$ should approach a Ricci-flat metric on the cone $C(H)$ with at least as many covariantly constant spinors as the original metric on $I$. These then translate directly into Killing spinors on $H$, as we should expect when comparing the amount of unbroken supersymmetry in the two interpretations $M \times{ }_{w} C(H)$ and $A d S \times H$ of our limiting compactification.

The great utility of Bär's theorem is that it can be combined with Berger's classification of holonomy groups $[45,46]$ to yield a classification of what supersymmetries are possible and what geometric structures are associated to them. We have collected the results of this classification into two tables, which we now explain.

For theories on $A d S_{p+2}$, the superconformal algebras must be particular ones from Nahm's list [47], with a bosonic part of the form $\mathfrak{o}(p+1,2) \oplus \mathfrak{g}$ (where $\mathfrak{g}$ is the $R$-symmetry algebra), and a fermionic part which is a representation of the bosonic algebra. The algebras which occur in our theories are labeled by the number of supersymmetries, and are listed in the middle column of Table 1 in the form

(bosonic algebra, fermionic representation).

The holonomy group on the cone $C(H)$ is a subgroup $\mathrm{Hol}_{C(H)} \subseteq S O(D-$ $p-1)$. The covariantly constant spinors are then determined as fixed elements of $\mathrm{Hol}_{C(H)}$ in the spin representation of $S O(D-p-1)$. Now for each fixed number $\mathcal{N}$ of spinors there is a group $\mathcal{H}_{\mathcal{N}} \subseteq S O(D-p-1)$ which fixes precisely that number of spinors. If the holonomy group of $C(H)$ is known, the amount of supersymmetry can then be determined by the condition $\mathrm{Hol}_{C(H)} \subseteq \mathcal{H}_{\mathcal{N}}, \mathrm{Hol}_{C(H)} \nsubseteq \mathcal{H}_{\mathcal{N}+1}$ (up to conjugacy).

The third column of Table 1 lists these possible "maximal holonomy" 


\begin{tabular}{|c|c|c|}
\hline $\mathcal{N}$ & Superconformal algebra & Maximal holonomy representation \\
\hline$(2,0)$ & $(\mathfrak{o}(6,2) \oplus \mathfrak{s p}(2),(\mathbf{8}, \mathbf{4}))$ & trivial 5-dim'l rep. \\
\hline$(1,0)$ & $(\mathfrak{o}(6,2) \oplus \mathfrak{s p}(1), \quad(\mathbf{8}, \mathbf{2}))$ & $(S U(2), \mathbf{2} \oplus \mathbf{2} \oplus \mathbf{1})$ \\
\hline 4 & $(\mathfrak{o}(4,2) \oplus \mathfrak{s u}(4), \quad(\mathbf{4}, \mathbf{4}) \oplus(\overline{4}, \overline{4}))$ & trivial 6-dim'l rep. \\
\hline 2 & $(\mathfrak{o}(4,2) \oplus \mathfrak{u}(2), \quad(\mathbf{4}, \mathbf{2}) \oplus(\overline{4}, \overline{2}))$ & $(S U(2), 2 \times(\mathbf{2} \oplus \mathbf{1}))$ \\
\hline 1 & $\left(\mathfrak{o}(4,2) \oplus \mathfrak{u}(1), \quad\left(\mathbf{4}, \mathbf{1}_{1}\right) \oplus\left(\overline{\mathbf{4}}, \mathbf{1}_{-1}\right)\right)$ & $(S U(3), \mathbf{3} \oplus \overline{\mathbf{3}})$ \\
\hline 8 & $(\mathfrak{o}(3,2) \oplus \mathfrak{s o}(8), \quad(4,8))$ & $\left(\mathbb{Z}_{2},\left\{ \pm \mathbb{1}_{8}\right\}\right)$ \\
\hline 6 & $(\mathfrak{o}(3,2) \oplus \mathfrak{s o}(6), \quad(4,6))$ & $\left(U(1), 4 \times\left(\mathbf{1}_{1} \oplus \mathbf{1}_{-1}\right)\right)$ \\
\hline 5 & $(\mathfrak{o}(3,2) \oplus \mathfrak{s o}(5),(4,5))$ & $(S U(2), 4 \times 2)$ \\
\hline 4 & $(\mathfrak{o}(3,2) \oplus \mathfrak{s o}(4),(4,4))$ & $(S U(2) \times S U(2), 2 \times((\mathbf{2}, \mathbf{1}) \oplus(\mathbf{1}, \mathbf{2})))$ \\
\hline 3 & $(\mathfrak{o}(3,2) \oplus \mathfrak{s o}(3),(4,3))$ & $(S p(2), \mathbf{4} \oplus \mathbf{4})$ \\
\hline 2 & $(\mathfrak{o}(3,2) \oplus \mathfrak{s o}(2), \quad(4,2))$ & $(S U(4), \mathbf{4} \oplus \overline{\mathbf{4}})$ \\
\hline 1 & $(\mathfrak{o}(3,2), 4)$ & $(\operatorname{Spin}(7), 8)$ \\
\hline
\end{tabular}

Table 1. Superconformal algebras and holonomy representations.

\begin{tabular}{|l|l|l|}
\hline Holonomy representation & Cone geometry & Horizon geometry \\
\hline trivial 5-dim'l rep. & $\mathbb{R}^{5}$ & $S^{4}$ \\
\hline trivial 6-dim'l rep. & $\mathbb{R}^{6}$ & $S^{5}$ \\
$(S U(3), \mathbf{3} \oplus \overline{\mathbf{3}})$ & Calabi-Yau threefold & Einstein-Sasaki 5-manifold \\
\hline trivial 8-dim'l rep. & $\mathbb{R}^{8}$ & $S^{7}$ \\
$(S p(2), \mathbf{4} \oplus \mathbf{4})$ & hyper-Kähler fourfold & 3-Sasaki 7-manifold \\
$(S U(4), \mathbf{4} \oplus \overline{\mathbf{4}})$ & Calabi-Yau fourfold & Einstein-Sasaki 7-manifold \\
$($ Spin $(7), \mathbf{8})$ & Spin(7)-manifold & nearly-parallel $G_{2}$-manifold \\
\hline
\end{tabular}

Table 2. Geometry of cones over smooth, simply-connected horizons. 
groups $\mathcal{H}_{\mathcal{N}}$, together with their $(D-p-1)$-dimensional representation ${ }^{5}$. Note that the number of spinors which is fixed can never be just one less than the dimension of the spinor representation (since fixing all but one of the spinors forces the last one to be fixed as well), but that there is otherwise no restriction on the $\mathcal{N}$ 's which occur.

Not all of the "maximal holonomy representations" can actually occur as holonomy groups; however, as we will see shortly, for each "maximal holonomy" group $\mathcal{H}_{\mathcal{N}}$ in the table there is some holonomy group $C(H)$ which lies in $\mathcal{H}_{\mathcal{N}}$ but not in $\mathcal{H}_{\mathcal{N}+1}$.

If the horizon manifold $H$ is smooth and simply-connected and the cone $C(H)$ is not flat, then the holonomy of the metric on $C(H)$ must act irreducibly in the sense that $\mathrm{Hol}_{C(H)}$ cannot be written as $G^{\prime} \times G^{\prime \prime}$ in such a way that the corresponding representation is a direct sum of a representation of $G^{\prime}$ with a representation of $G^{\prime \prime}$ [48]. This puts quite severe restrictions on the possible holonomies which can occur for such cones $C(H)$, limiting them to those in the first column of Table 2. The associated geometry for each of these holonomy groups is fairly well known; we list it in the second column of Table 2. Less familiar is the fact that the horizon manifolds also have a special geometric structure, determined by the existence of Killing spinors. These special geometric structures on the horizons are indicated in the final column of Table 2 .

We will give the precise definitions for these geometric structures on the horizon manifolds in an appendix; here, we simply note that each geometric structure is a direct consequence of the existence of a certain number of Killing spinors. There are some quite remarkable mathematical theorems which have been proven about these geometric structures $[49,50,44]$.

First, every 3-Sasaki manifold which is not a sphere has three distinguished vector fields generating an infinitesimal action of $\mathfrak{s u}(2)$ (as part of the definition). The theorem is that this can be integrated to an $S U(2)$ group action on the manifold, by isometries. Thus, in the case of a 3-Sasaki seven-manifold, the $R$-symmetry group $S U(2)$ or $S O(3)$ acts by isometries. (The center of $S U(2)$ will act faithfully in some cases, and trivially in other cases.)

Second, every Einstein-Sasaki manifold which is not 3-Sasaki has a distinguished vector field (again part of the definition). The theorem is that this can be integrated to a $U(1)$ action on the manifold. Thus, for an EinsteinSasaki five-manifold or seven-manifold, the $R$-symmetry group $U(1)$ or $S O(2)$ acts by isometries. Furthermore, for Einstein-Sasaki five-manifolds which

\footnotetext{
${ }^{5}$ In the third section of the table,the maximal holonomy groups appearing after the first line are $\operatorname{Spin}(2), \operatorname{Spin}(3), \ldots, \operatorname{Spin}(7)$ but we have given most of these a more familiar name.
} 
are not 3-Sasaki, the Einstein-Sasaki structure is unique; hence, so is the corresponding $U(1)$ action.

In the 3-Sasaki case, when the $S U(2)$ action is "regular", then the quotient by the $S U(2)$ action is a quaternionic Kähler manifold. The metric on the 3-Sasaki manifold can be recovered by using an appropriately scaled Hermitian metric on a quaternionic line bundle over the Kähler-Einstein manifold, restricted to the unit three-sphere bundle within that line bundle [51]. For simply-connected 3-Sasaki seven-manifolds with regular $S U(2)$ action, the conclusion is that the manifold must either be $S^{7}$ or the space $S U(3) / S_{1,1}^{1}=N(1,1)$ described in [52].

Similarly, in the Einstein-Sasaki case, when the $U(1)$ action is regular, the quotient by the $U(1)$ action is a Kähler-Einstein manifold of positive curvature, and the Einstein-Sasaki manifold can be recovered as the unit circle bundle in a certain Hermitian line bundle over the Kähler-Einstein manifold. For Einstein-Sasaki five-manifolds, this yields a complete classification for the case of regular $U(1)$-actions, since all positively curved Kähler-Einstein four-manifolds are known [53, 54]. The possible cases are as follows [49] (see also $[27,29])$.

1. $H / U(1) \cong \mathbb{C P}^{2}$ and either $H \cong S^{5}$ or $H \cong S^{5} / \mathbb{Z}_{3}$. These metrics are unique up to rescaling-in each case, they are induced by the "round", or constant curvature, metric on $S^{5}$.

2. $H / U(1) \cong \mathbb{C P}^{1} \times \mathbb{C P}^{1}$ and either $H \cong V_{4,2}$ or $H \cong V_{4,2} / \mathbb{Z}_{2}$, where $V_{4,2} \cong S O(4) / S O(2)$ is known as the "Stiefel manifold of framed twoplanes in four-space". (These horizons and the associated supergravity solutions have also been studied under the names $T^{11}$ and $T^{112}=$ $T^{11} / \mathbb{Z}_{2}$ [55].) The metric in each case is again unique up to scaling, due to a theorem of Bérard Bergery [56]. Writing $S O(4) \cong S^{3} \times S^{3}$, the metric on $H$ is induced from the product of round metrics on the three-spheres of equal volume.

3. $H / U(1)$ is a del Pezzo surface, the blowup of $\mathbb{C P}^{2}$ at $k$ points with $3 \leq k \leq 8$, and $H$ is a simply-connected $S^{1}$-bundle over this del Pezzo surface. Note that in this case, Kähler-Einstein metrics are known to exist on $H / U(1)[53,54]$, but they are not known explicitly.

In [49] it is also pointed out that for free group actions of $\Gamma \subset S U(3)$ on $S^{5}$ other than $\Gamma=\mathbb{Z}_{3}$, the $U(1)$ action is not regular. Thus, there are many other interesting possibilities for Einstein-Sasaki five-manifolds. 


\subsection{Detailed classification of holonomy groups}

We can obtain a more detailed classification of holonomy groups for M5branes by considering the connected component $\mathrm{Hol}^{0}$ of the holonomy group of the cone $C(H)$. The possible choices for this connected component again follow from Berger's classification [45]: it must be $S U(2)$ or trivial.

Suppose there is an M5-brane with $\mathrm{Hol}^{0}(C(H))=S U(2)$. After taking a finite unramified cover $\widetilde{H} \rightarrow H$ we get a cone $C(\widetilde{H})$ whose holonomy is reducible, with $C(\widetilde{H})$ locally the product of $\mathbb{R}$ (with a flat metric) and a fourdimensional space whose holonomy is $S U(2)$. By [48], $H$ cannot be smooth; on the other hand, the Gorenstein canonical singularities which can occur on $S U(2)$ spaces are only the ADE singularities, for which the holonomy is finite. The conclusion is that the holonomy group of $C(H)$ must be finite for M5-branes, with the horizon taking the form $H=S^{4} / \Gamma$ for some finite group $\Gamma \subset S U(2)$. Notice that $\Gamma$ always fixes two points, so that if the holonomy is nontrivial (that is, if we get an $\mathcal{N}=(1,0)$ theory rather than an $\mathcal{N}=(2,0)$ theory) then the horizon is singular.

Similarly, in the case of D3-branes, the possible choices for $\operatorname{Hol}^{0}(C(H))$ are $S U(3), S U(2)$ and trivial [45], and we find several possibilities for the holonomy (assuming that there is some supersymmetry).

1. If $\operatorname{Hol}^{0}(C(H))=S U(3)$ then $\operatorname{Hol}(C(H))=S U(3)$ and we get $\mathcal{N}=1$ supersymmetry. We will see several examples of this case later in the paper. The horizon is an Einstein-Sasaki five-manifold with a $U(1)$ action which geometrically realizes the $\mathcal{N}=1 R$-symmetry group by isometries of $H$.

2. The case $\operatorname{Hol}^{0}(C(H))=S U(2)$ is unlikely to occur. As in the case of M5-branes, an unramified cover of $H$ must have reducible holonomy, and so by [48] $H$ cannot be smooth. The singularities on $H$ all derive from the complex codimension two singularities on $C(H)$, which must again be ADE singularities. However, since we now have a family of ADE singularities we cannot immediately conclude that the holonomy is broken to a finite group. (It seems likely that it will be. ${ }^{6}$ )

3. If $\mathrm{Hol}^{0}(C(H))$ is trivial, then $\operatorname{Hol}(C(H))=\Gamma$ is a finite group, with $H=S^{5} / \Gamma$. If $\Gamma$ is not trivial but $\Gamma \subset S U(2)$, then $H$ has singularities along a circle and we get $\mathcal{N}=2$ supersymmetry. (This is consistent with the discussion in case 2.) In this case, the horizon has a natural

\footnotetext{
${ }^{6}$ This conclusion is based on our analysis of the "suspended pinch point" below. That singularity is associated to a family of ADE singularities, but its holonomy does not appear to break to a finite group. However, due to complications within the family of ADE singularities we in fact get $\mathcal{N}=1$ supersymmetry for this example and we suspect that the holonomy is all of $S U(3)$ in this case. Unfortunately, the metric is not known explicitly.
} 
$S U(2) \times U(1)$ isometry group, again geometrically realizing the $R$ symmetries.

If $\Gamma \subset S U(3), \Gamma \not \subset S U(2)$, then we get $\mathcal{N}=1$ supersymmetry (and again have a $U(1)$ action which realizes the $R$-symmetry group). Both isolated and non-isolated singularities can occur in this latter case. (For a classification of non-isolated singularities with $\Gamma$ a cyclic group, see [57].)

An analysis similar to this could also be carried out for M2-branes, but it is quite involved so we shall defer it to some future occasion on which a more detailed studied of M2-branes is made.

\section{M2-branes and M5-branes}

To study M2-branes at singularities following our program, we would need some kind of classification of the singularities which can occur. In the case of general $\mathcal{N}=1$ theories in which the holonomy group of the cone is $\operatorname{Spin}(7)$, there do not appear to be any techniques for making such a classificationthe geometry of degenerate $\operatorname{Spin}(7)$ manifolds is an almost completely unexplored subject. ${ }^{7}$ Of course, one could attack this from the other point of view, and attempt to classify all Einstein seven-manifolds with a "nice" three-form, but this would also appear to be quite difficult. Many examples of such manifolds are known (see [52] for a list of known examples circa $1985^{8}$ ). A third approach-equally difficult-would be to try to classify all families of $\mathcal{N}=1$ conformal theories in three dimensions with a large $N$ limit.

Even for theories with $\mathcal{N}=2$ supersymmetry, the singularities involved are Gorenstein canonical singularities of Calabi-Yau fourfolds, and their complete classification seems an extremely challenging problem. ${ }^{9}$ We hope to return to a more detailed study of some classes of these singularities in a future work; for the present, we will focus on a few examples.

\subsection{M2-branes at orbifolds}

First, let us note that a variety of supersymmetries can be obtained just from orbifold constructions-horizons in $\mathbb{C}^{4} / \Gamma$-even assuming that the action of $\Gamma$ on $S^{7}$ is free. When the group $\Gamma$ is cyclic, there is a characterization

\footnotetext{
${ }^{7}$ There are some examples known, however-the first constructions of complete [58] and of compact [59] Spin(7)-manifolds are both related to degenerations of the sort considered here.

${ }^{8}$ Additional examples have recently been discussed in [34].

${ }^{9}$ See $[60]$ for a hint of some of the difficulties.
} 
from algebraic geometry - the singularity is said to be "terminal" - which singles out the freely acting $\Gamma$ 's which are contained in $S U(2) \times S U(2)$ [61]. In fact, any such group is generated by $\operatorname{diag}\left(e^{2 \pi i / k}, e^{-2 \pi i / k}, e^{2 \pi i a / k}, e^{-2 \pi i a / k}\right)$ for some relatively prime integers $a$ and $k$. If $a=1, k=2$, we get $\mathcal{N}=8$ supersymmetry with horizon manifold $\mathbb{R P}^{7}[62,63]$. If $a= \pm 1, k>2$ then $\Gamma$ lies in $U(1)$ and we get $\mathcal{N}=6$ supersymmetry [19]. ${ }^{10}$ If $a \neq \pm 1$, the resulting theory has $\mathcal{N}=4$ supersymmetry. All of these singularities have the interesting property of having neither (complex structure) deformations [65], nor (Calabi-Yau) resolutions. ${ }^{11}$

As a slight variant of this example, if we take $\Gamma$ to be a binary dihedral group, so that $\mathbb{C}^{2} / \Gamma$ is a $D_{k}$ singularity, and diagonally embed $\Gamma$ into $S U(2) \times S U(2)$, then the corresponding orbifold theory has $\mathcal{N}=5$ supersymmetry. ${ }^{10,12}$. A similar construction produces $\mathcal{N}=5$ supersymmetry from $E_{6}, E_{7}$, and $E_{8}$ singularities. Many other orbifolds are possible, with both free and non-free actions on $S^{7}$, leading to a variety of different amounts of supersymmetry.

\subsection{M2-branes and exotic spheres}

Another type of example is produced by hypersurface singularities. For instance, the hypersurface in $\mathbb{C}^{5}$ defined by

$$
v^{2}+w^{2}+x^{2}+y^{3}+z^{6 k-1}=0
$$

has an isolated Gorenstein canonical singularity at the origin for any integer $k$. These singularities can be realized on Calabi-Yau fourfolds for low values of $k$-e.g., on a degree six hypersurface in $\mathbb{C P}^{5}$ in the case of $k=1$. The singularities (3.1) were studied many years ago by Brieskorn [68, 69], who showed that the corresponding horizon manifold is topologically isomorphic to $S^{7}$, but not in general diffeomorphic to $S^{7}$-in particular, in the case $k=1$ it is not diffeomorphic to $S^{7}$. These horizons thus give examples of exotic seven-spheres, ${ }^{13}$ and we should expect to be able to put M2-branes at the relevant singularities and find Einstein metrics on the exotic spheres.

The set of diffeomorphism classes of topological seven-spheres is known to form a group of order 28 [72], and Brieskorn showed that the horizons for the hypersurfaces (3.1) with $k=1,2, \ldots, 28$ include all of these exotic spheres.

\footnotetext{
${ }^{10}$ The standard lore in the supergravity literature which excludes $\mathcal{N}=5,6$ supersymmetry from $A d S_{4} \times X^{7}$ compactifications applies only when $X^{7}$ is simply connected [64].

${ }^{11}$ Any Calabi-Yau resolution must have a "small" exceptional set and so by [66] must be toric; but it's easy to show that these singularities do not have toric Calabi-Yau resolutions.

${ }^{12} \mathcal{N}=5$ supergravity theories were constructed in [67]

${ }^{13}$ For earlier discussions of exotic seven-spheres in the physics literature, see $[70,71]$. The reappearance of this mathematical topic in physics-in the context of string theory-was predicted by S. Shenker in 1996 .
} 
For addressing the problem of finding Einstein metrics on these spaces, Milnor's original description [73] of exotic seven-spheres as $S^{3}$-bundles over $S^{4}$ is probably more useful. In fact, Gromoll and Meyer used that description to construct a metric with nonnegative sectional curvature on one of the exotic seven-spheres [74] (one of the generators of the group), but it is apparently still unknown whether or not any of the exotic seven-spheres admit Einstein metrics.

The Gromoll-Meyer metric has an isometry group of $S O(3) \times O(2)$; moreover, any exotic seven-sphere has a differentiable action of $S O(3) \times O(2)$ [75], which is the largest its isometry group could be for any metric. It is unclear whether we should expect the $S O(3)$ or the $S O(2)$ factor to play the role of the $R$-symmetry group, i.e., whether we should expect $\mathcal{N}=3$ or $\mathcal{N}=2$ supersymmetry for the resulting superconformal theories. We expect at least $\mathcal{N}=2$ supersymmetry since this is coming from a singularity on a CalabiYau manifold.

\subsection{M5-branes}

Turning now to M5-branes, as we observed in section 2.1, in order to obtain a model with $\mathcal{N}=(1,0)$ supersymmetry we must consider a horizon of the form $H=S^{4} / \Gamma$ with $\Gamma \subset S U(2)$ a finite group. In the global context of the Horrava-Witten heterotic string compactified on $\left(S^{1} / \mathbb{Z}_{2}\right) \times K 3$, this means that we must allow the $\mathrm{K} 3$ surface to acquire a rational double point at $Q$-so that $\left(S^{1} / \mathbb{Z}_{2}\right) \times K 3$ is singular all along $S^{1} \times\{Q\}$, choose a point $P \in S^{1} \times\{Q\}$, and place $N$ M5-branes at $P$. It is important that the branes be located at the same position in the eleventh dimension.

In order to find a description of the resulting theory, it is useful to employ the dual model in $F$-theory, worked out in [76] with some important additions in [77]. ${ }^{14}$ We will briefly sketch this, deferring details to a companion publication [11]. Our task is to place $N$ small instantons at $Q$ and study the phase transition to the model in which the corresponding M5-branes have left the boundary, keeping all $N$ of the branes located at the same position in the eleventh dimension. In [76] it was explained how to do this, by studying the behavior of the discriminant locus in the $F$-theory dual model. Placing $N$ small instantons at a singular point $Q$ creates a singularity in the base of the elliptic fibration of the $F$-theory model, which must then be resolved.

The sizes of the exceptional divisors in the resolution translate to distances among the M5-branes and the boundary HW9-branes in the HořavaWitten description. In order to keep the M5-branes together in the eleventh dimension, it is thus necessary to blow down all but one of the exceptional

\footnotetext{
${ }^{14}$ The six-dimensional $E 8 \times E 8$ theories studied in $[76,77]$ also have an interesting duality relating them to models with $S O(32)$ heterotic 5-branes [78].
} 
divisors. (The one remaining is the one adjacent to the boundary, which measures the distance between the collective M5-brane location and the boundary.) This sends all gauge groups appearing in the effective field theory to strong coupling. A precise description of the strong-coupling fixed points thus obtained can be found in $[76,77,11]$.

Note that it will be difficult to make direct checks of the generalized conjecture in this case due to the presence of orbifold singularities in the horizon. We do not yet know how to treat such singularities in M-theory. (But see $[79,80]$ for orientifolds.)

This proposal for a dual field theory for M5-branes at orbifolds differs from one which has previously been studied in the literature $[16,21]$. In that earlier proposal, the $N$ M5-branes were placed at different locations in the eleventh dimension.

From the global analysis above, it is clear that there is one further type of point which must be considered in order to have a complete story for M5branes: the transition points at which the M5-branes are placed at a singular point, but with that point located on the HW9-brane. We do not currently have a proposal for the effective theory in such cases. The analysis of these transition points will presumably involve the type of orientifold description pioneered by Berkooz [13] for M5-branes at the HW9-brane at a smooth point of K3 (see also [81]).

\section{Gorenstein canonical singularities in three com- plex dimensions}

For the remainder of this paper, we focus our attention on D3-branes in fourdimensional $F$-theory models, which we regard as type IIB string compactifications on backgrounds which include D7-branes. We restrict our attention to singular points of the compactifying space away from the D7-branes. (A study of what can happen at the D7-branes was initiated in $[8,9,10]$.) The relevant singularities are the Gorenstein canonical singularities in three complex dimensions. When we place $N$ D3-branes at such a singularity, we will find a four-dimensional theory with $\mathcal{N}=1$ or $\mathcal{N}=2$ supersymmetry. ${ }^{15}$

Gorenstein canonical singularities in complex dimension three have been studied extensively in the algebraic geometry literature (see [83] for the definitions and earliest results, and [84] for a thorough review). There is a kind of inductive classification of these singularities, obtained by blowing them up one step at a time; there are also detailed descriptions available for special

\footnotetext{
${ }^{15} \mathrm{~A}$ general analysis of such theories and their connection to $A d S$ supergravity theories was given in [82].
} 
classes of these singularities such as the toric ones. We will consider in detail two special types of Gorenstein canonical singularities.

\subsection{Singularities resolved by only one blowup}

Gorenstein canonical threefold singularities which arise by contracting a single extremal ray (i.e., those which can be resolved with a single blowup) can be classified fairly easily. These singularities form three types [85], depending on the behavior of the contraction mapping: we can have curves contracting to points (type I), a surface contracting to a point (type II), or a surface contracting to a curve (type III). If the complex structure is generic, these singularities can be more explicitly described as follows:

- type I: a collection of $n \geq 1$ smooth $\mathbb{C} P^{1}$ 's, each with normal bundle $\mathcal{O}(-1) \oplus \mathcal{O}(-1)$, is contracted to $n$ "conifold" points

- type III: a rational ruled surface whose ruling has $n \geq 0$ degenerate fibers is contracted to a curve

- type II: a del Pezzo surface of Picard number $n+2$ is contracted to a point $(-1 \leq n \leq 7)$

This same list of singularities is familiar from another application. When Mtheory is compactified on a Calabi-Yau threefold with such a singularity [86, 87], there is a five-dimensional field theory associated to the singularity which decouples from gravity [88]. Most of these field theories can be described in gauge theory terms, as follows:

- type I: $U(1)$ gauge theory with $n \geq 1$ "electrons", which can also be described as $n$ D8-branes in Type IA string theory on $S^{1} / \mathbb{Z}_{2}$

- type III: $S U(2)$ gauge theory with $n \geq 0$ "quarks", which can also be described as $n$ D8-branes at an orientifold plane in Type IA string theory on $S^{1} / \mathbb{Z}_{2}$

- type II: strong-coupling limit of $S U(2)$ gauge theory with $n$ "quarks" $(0 \leq n \leq 7)$. There is also a variant with a $\mathbb{Z}_{2} \theta$-angle when $n=0$, and a theory corresponding to $n=-1$ which cannot be obtained as a strong-coupling limit of a gauge theory.

(In [86], these theories were labeled by their global symmetry groups: $A_{n-1}$, $D_{n}$ and $E_{n+1}$ in the three cases above.) Compactifying on a circle to obtain a IIA theory in four dimensions, the type I singularities correspond to the familiar conifold points at which there are massless hypermultiplets [89] and 
a conifold transition [90]; the type III singularities correspond to enhanced gauge symmetry and can also exhibit a transition [91, 92]; and the type II singularities are associated to "extremal transitions" whose physics is less well understood.

Returning to the discussion at hand, when we consider $N$ of the spacefilling D3-branes present in an $F$-theory model whose base has acquired such a singularity, and move the branes to the singular locus, there are several things which can happen. In the case of a type I contraction, the compactifying space has several singular points but they are all locally isomorphic to a conifold singularity, so we must study branes at a conifold to cover this case. In the case of a type III contraction, the singularity is not isolated, but there are only two isomorphism classes of local singularity types which occur, corresponding to the contraction at a nonsingular fiber of the ruling on the surface (the generic case), or at a singular fiber. In the case of a type II contraction, there is only one singular point so there is no ambiguity about where to place the branes.

In short, to cover all of the cases above we must consider the following kinds of singular points and their associated horizons:

- type I: a conifold point with local equation $x y=z t$, whose horizon is $H_{c o n}=V_{4,2}=\left(S^{3} \times S^{3}\right) / U(1)[93]$.

- type III generic: a quotient singularity $\mathbb{C}^{3} / \mathbb{Z}_{2}$ (in which $\mathbb{Z}_{2}$ acts on only two of the three complex coordinates) with local equation $x y=z^{2}$, whose horizon is $S^{5} / \mathbb{Z}_{2}$

- type III special: a "suspended pinch point" with local equation $x y=$ $z^{2} t$, whose horizon is a circle bundle over a product of weighted projective spaces $\mathbb{W} \mathbb{C P}^{1,2} \times \mathbb{W} \mathbb{C P}^{1,2}$ (as we will see in section 4.3)

- - type II: the complex cone over a del Pezzo surface, whose horizon is a circle bundle over the del Pezzo surface. The complex cone and the circle bundle are built from the anti-canonical line bundle of the del Pezzo surface.

There are two kinds of del Pezzo surfaces: the surface $\mathbb{F}_{0}=\mathbb{C P}^{1} \times \mathbb{C P}^{1}$, or the blowup of $\mathbb{C P}^{2}$ at $k$ points with $0 \leq k \leq 8$. Note that the circle bundle in the anti-canonical bundle of $\mathbb{C P}^{2}$ yields $S^{5} / \mathbb{Z}_{3}$ as its horizon manifold, and the circle bundle in the anti-canonical bundle of $\mathbb{F}_{0}$ yields $V_{4,2} / \mathbb{Z}_{2}$ as its horizon manifold.

Thus, we find an almost perfect match between isolated Gorenstein canonical singularities obtained by contracting a single extremal ray (classified via algebraic geometry), and Einstein-Sasaki five-manifolds with a 
regular $U(1)$-action (classified via differential geometry). There is a small puzzle, however: the cones over del Pezzo surfaces which are the blowups of $\mathbb{C P}^{2}$ at one or two points are missing form the list of regular EinsteinSasaki horizons. It is not even known if there exist Einstein-Sasaki metrics on these spaces. One possibility is that they have such metrics, but-like many orbifolds - the $U(1)$ action is not regular. Another possibility is that the limiting metrics have a more complicated asymptotic behavior than our basic ansatz (1.16). We note in passing that these same two singularities exhibited exceptional behavior in the earlier application to construction of five-dimensional field theories [86]: on the one hand, these were the only two whose deformation space has extra first-order directions that do not extend to full deformations [94, 95]; on the other hand, the global symmetry groups of the corresponding five-dimensional field theories were the only two which are not semisimple groups-each one them has a $U(1)$ factor. (These are the groups denoted $\widetilde{E}_{1}$ and $E_{2}$ in [86].)

\subsection{Toric singularities}

Another class of Gorenstein canonical singularities which can be easily classified is the class of toric singularities; ${ }^{16}$ this includes all singularities of the form $\mathbb{C}^{n} / \Gamma$ for finite abelian groups $\Gamma \subset U(n)$. According to Reid's criterion [83], the data needed to define a toric Gorenstein canonical singularity of complex dimension $n$ is a convex polygon in $\mathbb{R}^{n-1}$ whose vertices have integer coordinates. Given that data, one construction of the singularity is as follows: ${ }^{17}$ let $v_{1}, \ldots, v_{k}$ be all of the vectors in $\mathbb{R}^{n-1}$ which have integer coordinates, and which lie in either the interior or the boundary of the polygon. Consider the linear relations among these vectors

$$
\sum_{j=1}^{k} Q^{j} v_{j}=0
$$

with integer coefficients $Q^{j}$, subject to

$$
\sum_{j=1}^{k} Q^{j}=0 .
$$

Let $\vec{Q}_{1}, \ldots, \vec{Q}_{k-n}$ be a basis for the set of all such relations, and use the matrix $\left(Q_{i}^{j}\right)$ to specify the charges in a representation of $U(1)^{k-n}$ on $\mathbb{C}^{k}$. The singular space is then the symplectic reduction $\mathbb{C}^{k} / / U(1)^{k-n}$, using the moment map centered at the origin. (This mathematical construction should be familiar from studies of $\mathcal{N}=(2,2)$ abelian gauge theory in two dimensions, with gauge group $U(1)^{k-n}$ and chiral matter in the representation

\footnotetext{
${ }^{16}$ For a review of toric geometry for physicists, see [96, 97]

${ }^{17}$ As we will see shortly, there are other possible constructions.
} 
specified by $\left(Q_{i}^{j}\right)$ and no Fayet-Iliopolous terms-see [98, 97], for example.) Clearly, this singularity doesn't change if we shift the location of the polygon by adding an integer vector to it.

Varying the center of the moment map then produces partial or complete resolutions of this singularity. ${ }^{18}$ (In the $\mathcal{N}=(2,2)$ gauge theory, this corresponds to turning on certain Fayet-Iliopoulos $D$-terms.) The location of the center of the moment map determines the sizes of holomorphic curves within the exceptional set of the resolution, i.e., the blowup moduli of the singularity. Particular values of these moduli will correspond to partial rather than complete resolutions. In general, any convex sub-polygon can represent the residual singularity in one of the partial resolutions.

An alternate description of toric singularities is often very useful: they can be described by a collection of polynomial equations of the form "one monomial equals another monomial." To produce such a description from the data above, introduce homogeneous coordinates $x_{1}, \ldots, x_{k}$, and find a minimal generating set $z_{1}, \ldots z_{\ell}$ for

$$
\left\{U(1)^{k-n} \text {-invariant monomials } z=x_{1}^{a_{1}} \cdots x_{k}^{a_{k}} \mid a_{i} \geq 0\right\} .
$$

The $z_{j}$ 's give coordinates in the ambient space of the toric singularity, and the polynomial equations relating various monomials in the $z_{j}$ 's can be found in a straightforward manner.

For example, if we let $\Gamma=\mathbb{Z}_{m} \times \mathbb{Z}_{m}$ act on $\mathbb{C}^{3}$ via the generators $\operatorname{diag}\left(e^{2 \pi i / m}, e^{-2 \pi i / m}, 1\right)$ and $\operatorname{diag}\left(e^{2 \pi i / m}, 1, e^{-2 \pi i / m}\right)$, then the quotient singularity $\mathbb{C}^{3} / \Gamma$ is described by the polygon with vertices $(0,0),(m, 0)$ and $(0, m)$. The set of vectors $v_{j}$ consists of all integer vectors $(k, \ell)$ with $k \geq 0$, $\ell \geq 0$, and $k+\ell \leq m$. Notice that the convex polygon associated to any three-dimensional toric Gorenstein canonical singularity can be embedded as a sub-polygon of $\langle(0,0),(m, 0),(0, m)\rangle$ for some $m$, after shifting its location by an appropriate vector.

\section{Partial resolutions of the $\mathbb{Z}_{2} \times \mathbb{Z}_{2}$ orbifold}

To be more explicit in the case of $\mathbb{Z}_{2} \times \mathbb{Z}_{2}$, let us label the vectors as

$$
v_{0}=(0,0) \quad v_{1}=(2,0) \quad v_{2}=(0,2) \quad w_{0}=(1,1) \quad w_{1}=(0,1) \quad w_{2}=(1,0) .
$$

(These vectors are illustrated in Figure 1(a).) A basis for the relations subject to (4.2) is then given by

$$
v_{0}+w_{0}-\dot{w}_{1}-w_{2}=0, \quad v_{1}-w_{0}+w_{1}-w_{2}=0, \quad v_{2}-w_{0}-w_{1}+w_{2}=0 .
$$

\footnotetext{
${ }^{18} \mathrm{By}$ including all integer vectors $v_{j}$ lying within the polygon, we have included all possible toric blowups with a non-vanishing holomorphic $n$-form.
} 
We introduce homogeneous coordinates $x_{0}, x_{1}, x_{2}, y_{0}, y_{1}, y_{2}$ and consider the $U(1)^{3}$ action determined by (4.5); it has charge matrix

$$
\left(\begin{array}{cccccc}
x_{0} & x_{1} & x_{2} & y_{0} & y_{1} & y_{2} \\
1 & 0 & 0 & 1 & -1 & -1 \\
0 & 1 & 0 & -1 & 1 & -1 \\
0 & 0 & 1 & -1 & -1 & 1
\end{array}\right)
$$

The moment map, using arbitrary $D$-terms, is therefore

$$
\begin{aligned}
& \left|x_{0}\right|^{2}+\left|y_{0}\right|^{2}-\left|y_{1}\right|^{2}-\left|y_{2}\right|^{2}=\zeta_{1} \\
& \left|x_{1}\right|^{2}-\left|y_{0}\right|^{2}+\left|y_{1}\right|^{2}-\left|y_{2}\right|^{2}=\zeta_{2} \\
& \left|x_{2}\right|^{2}-\left|y_{0}\right|^{2}-\left|y_{1}\right|^{2}+\left|y_{2}\right|^{2}=\zeta_{3}
\end{aligned}
$$

Another equation which follows from these is

$$
\left|x_{0}\right|^{2}+\left|x_{1}\right|^{2}-2\left|y_{2}\right|^{2}=\zeta_{1}+\zeta_{2}
$$
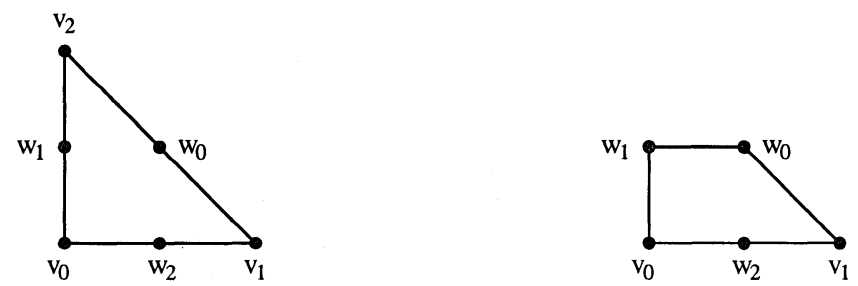

Figure 1(a). $\mathbb{Z}_{2} \times \mathbb{Z}_{2}$ orbifold. Figure 1(b). Suspended pinch point.

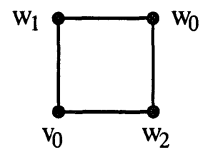

Figure 1(c). Conifold.

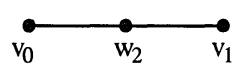

Figure $1(\mathrm{~d}) . \mathbb{Z}_{2}$ orbifold.

When $\zeta_{1}, \zeta_{2}$ and $\zeta_{3}$ are generic, the symplectic reduction is smooth. There are several singularity types which can be obtained for special values of the $\zeta_{i}$. These are illustrated in Figure 1, and described as follows. Each singularity is associated to a subgroup $U(1)^{k}$ of $U(1)^{3}$ with the property that at least $3-k$ of the homogeneous coordinates are uncharged under $U(1)^{k}$ (and the convex hull of the corresponding vectors spans the space). The singular space is then described by symplectic reduction of the space $\mathbb{C}^{k+3}$ spanned by the remaining $k+3$ homogeneous coordinates by $U(1)^{k}$. For each partial resolution, we have listed in Table 3 the condition on $D$-term coefficients which selects out $U(1)^{k}$, and the corresponding charge matrix on the remaining $k+3$ homogeneous coordinates. The polygons corresponding to the relevant homogeneous coordinates are illustrated in Figure 1. 


\begin{tabular}{|l|l|lll|}
\hline & & $\left(\begin{array}{cccccc|}x_{0} & x_{1} & x_{2} & y_{0} & y_{1} & y_{2} \\
1 & 0 & 0 & 1 & -1 & -1 \\
0 & 1 & 0 & -1 & 1 & -1 \\
0 & 0 & 1 & -1 & -1 & 1\end{array}\right)$ \\
\hline suspended pinch point & $\zeta_{1}=\zeta_{2}=\zeta_{3}=0$ & $\left(\begin{array}{ccccc}x_{0} & x_{1} & y_{0} & y_{1} & y_{2} \\
1 & 0 & 1 & -1 & -1 \\
0 & 1 & -1 & 1 & -1\end{array}\right)$ \\
\hline conifold & $\zeta_{1}=0$ & $\left(\begin{array}{cccc}x_{0} & y_{0} & y_{1} & y_{2} \\
1 & 1 & -1 & -1\end{array}\right)$ \\
\hline & & $\left(\begin{array}{cccc}x_{0} & x_{1} & x_{2} & y_{2} \\
1 & 1 & 0 & -2\end{array}\right)$ \\
\hline $\mathbb{Z}_{2}$ orbifold & $\zeta_{1}+\zeta_{2}=0$ &
\end{tabular}

Table 3. Charge matrices for partial resolutions of the $\mathbb{Z}_{2} \times \mathbb{Z}_{2}$ orbifold.

\begin{tabular}{|c|c|c|c|}
\hline $\mathbb{Z}_{2} \times \mathbb{Z}_{2}$ orbifold & $\begin{array}{l}z_{1}=x_{0}^{2} y_{1} y_{2} \\
z_{3}=x_{2}^{2} y_{0} y_{1}\end{array}$ & $\begin{array}{l}z_{2}=x_{1}^{2} y_{0} y_{2} \\
z_{4}=x_{0} x_{1} x_{2} y_{0} y_{1} y_{2}\end{array}$ & $z_{1} z_{2} z_{3}=z_{4}^{2}$ \\
\hline suspended pinch point & $\begin{array}{l}z_{1}=x_{0}^{2} y_{1} y_{2}, \\
z_{3}=x_{1}^{2} y_{0} y_{1}\end{array}$ & $\begin{array}{l}z_{2}=x_{0} x_{1} y_{2} \\
z_{4}=y_{0} y_{1}\end{array}$ & $z_{1} z_{3}=z_{2}^{2} z_{4}$ \\
\hline conifold & $\begin{array}{l}z_{1}=x_{0} y_{1} \\
z_{3}=y_{0} y_{1}\end{array}$ & $\begin{array}{l}z_{2}=x_{0} y_{2} \\
z_{4}=y_{0} y_{2}\end{array}$ & $z_{1} z_{4}=z_{2} z_{3}$ \\
\hline $\mathbb{Z}_{2}$ orbifold & $\begin{array}{l}z_{1}=x_{0}^{2} y_{2} \\
z_{3}=x_{1}^{2} y_{2}\end{array}$ & $\begin{array}{l}z_{2}=x_{0} x_{1} y_{2} \\
z_{4}=x_{2}\end{array}$ & $z_{1} z_{3}=z_{2}^{2}$ \\
\hline
\end{tabular}

Table 4. Invariant monomials and equations for partial resolutions of the $\mathbb{Z}_{2} \times \mathbb{Z}_{2}$ orbifold. 
For each of these singularities, we can compute the $U(1)^{k}$-invariant monomials and the equations they satisfy, giving the algebraic realization of the singularity. This is carried out in Table 4. The equations enable us to recognize some of these singularities as familiar ones, such as the $\mathbb{Z}_{2}$ orbifold $z_{1} z_{3}=z_{2}^{2}$ ("type III generic" in section 4.1), the conifold singularity $z_{1} z_{4}=z_{2} z_{3}$ ("type I" in section 4.1), and the "suspended pinch point"19 $z_{1} z_{3}=z_{2}^{2} z_{4}$ ("type III special" in section 4.1).

The presentations of these singularities which we have given include all possible "crepant" toric blowups by varying the $D$-terms. (The crepant blowups are the ones for which the pullback of the holomorphic $n$-form near the singularity is non-vanishing.) However, in many cases there is a more "efficient" description of the singularity, involving fewer fields and a smaller group. In terms of the convex polygon, the most efficient description is obtained by using only the vertices of the polygon and omitting the other vectors $v_{j}$. One modification which must be made to the construction is that if the vertices do not span a primitive sublattice of $\mathbb{Z}^{n}$, then after performing the symplectic reduction we must implement a further orbifold by a finite group $G$ (the torsion subgroup of the quotient of $\mathbb{Z}^{n}$ by the span of the $v_{j}$ 's).
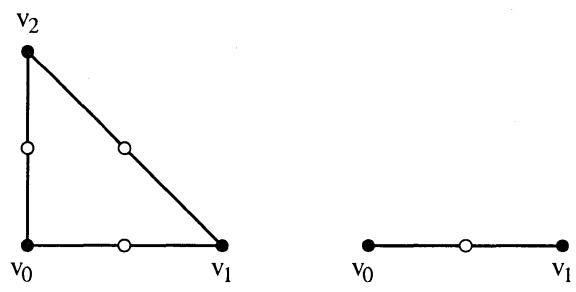

Figure 2. The combinatorial data for $\mathbb{Z}_{2} \times \mathbb{Z}_{2}$ and $\mathbb{Z}_{2}$ orbifolds.

For two of our examples-the $\mathbb{Z}_{2} \times \mathbb{Z}_{2}$ orbifold and $\mathbb{Z}_{2}$ orbifold-this procedure leads back directly to an orbifold description. The combinatorics for the most efficient description are shown in Figure 2. In the first case, the invariants for the $\mathbb{Z}_{2} \times \mathbb{Z}_{2}$ action $\left(x_{0}, x_{1}, x_{2}\right) \mapsto\left((-1)^{a+b} x_{0},(-1)^{a} x_{1},(-1)^{b} x_{2}\right)$ are $z_{1}=x_{0}^{2}, z_{2}=x_{1}^{2}, z_{3}=x_{2}^{2}$ and $z_{4}=x_{0} x_{1} x_{2}$, with equation $z_{1} z_{2} z_{3}=z_{4}^{2}$, so we recover our original description. In the second case, the invariants for the $\mathbb{Z}_{2}$ action $\left(x_{0}, x_{1}, x_{2}\right) \mapsto\left(-x_{0},-x_{1}, x_{2}\right)$ are $z_{1}=x_{0}^{2}, z_{2}=x_{0} x_{1}, z_{3}=x_{1}^{2}$, and $z_{4}=x_{2}$ with equation $z_{1} z_{3}=z_{2}^{2}$ and we again recover our original description.

\footnotetext{
${ }^{19}$ This singularity is the suspension, in the sense of singularity theory, of the pinch points which occur in the theory of ordinary singularities of surfaces [99].
} 

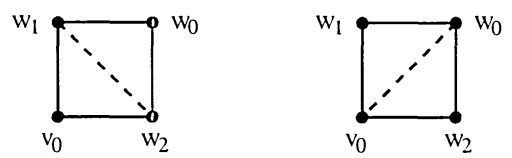

Figure 3. The two resolutions of the conifold.

For the other two examples - the suspended pinch point and the conifoldthe most efficient description involves a single $U(1)$. In the case of the conifold, this is the description which we have already given. Varying the $D$-term for the $U(1)$ produces the two small resolutions of the conifold, related by a flop, as illustrated in Figure 3.
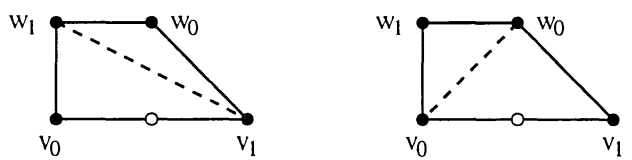

Figure 4. The partial resolutions of the suspended pinch point in its minimal presentation.

In the case of the suspended pinch point, the efficient description involves the vectors $v_{0}, v_{1}, w_{1}$ and $w_{2}$, related by

$$
v_{0}-v_{1}+2 w_{0}-2 w_{1}
$$

which leads to the charge matrix

$$
\left(\begin{array}{cccc}
x_{0} & x_{1} & y_{0} & y_{1} \\
1 & -1 & 2 & -2
\end{array}\right)
$$

The invariants monomials are $z_{1}=x_{0}^{2} y_{1}, z_{2}=x_{0} x_{1}, z_{3}=x_{1}^{2} y_{0}$ and $z_{4}=$ $y_{0} y_{1}$ with equation $z_{1} z_{3}=z_{2}^{2} z_{4}$ so that we recover our original description. Varying the $D$-term for the $U(1)$ produces two small partial resolutions, with exceptional set $\mathbb{C P}^{1}$ but still having singularities along a curve. The toric data for these is illustrated in Figure 4.

\section{Complex cones over del Pezzo surfaces}

Two of the complex cones over del Pezzo surfaces (i.e., singularities of "type II" in the terminology of section 4.1) have particularly simple toric descriptions: they are the complex cones over $\mathbb{C P}^{2}$ and over $\mathbb{F}_{0}$. The corresponding convex polygons are illustrated in Figure 5; they involve the vectors

$v_{0}=(0,0) \quad w_{0}=(1,1) \quad w_{1}=(0,1) \quad w_{2}=(1,0) \quad u_{1}=(2,1) \quad u_{2}=(1,2)$. 
The relations subject to (4.2) are generated by

$$
v_{0}+u_{1}+u_{2}-3 w_{0}=0
$$

for the complex cone over $\mathbb{C P}^{2}$, and by

$$
w_{1}+u_{1}-2 w_{0}=0, \quad w_{2}+u_{2}-2 w_{0}=0
$$

for the complex cone over $\mathbb{F}_{0}$.
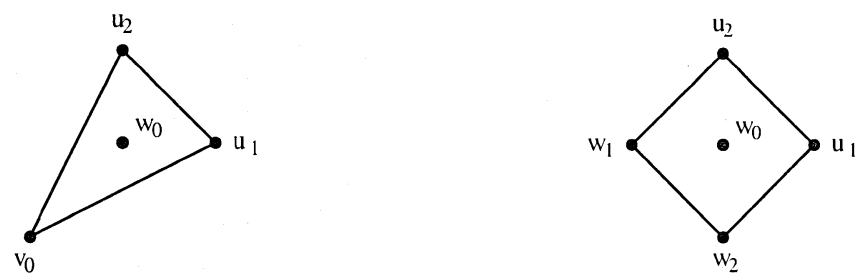

Figure 5(a). Complex cone over $\mathbb{C P}^{2}$. Figure 5(b). Complex cone over $\mathbb{F}_{0}$.

In the case of the complex cone over $\mathbb{C P}^{2}$, the singularity can be described by means of homogeneous coordinates $x_{0}, y_{0}, z_{1}, z_{2}$ with $U(1)$ action

$$
\left(\begin{array}{cccc}
x_{0} & y_{0} & z_{1} & z_{2} \\
1 & -3 & 1 & 1
\end{array}\right) .
$$

The moment map with $D$-term $\zeta$ is given by

$$
\left|x_{0}\right|^{2}-3\left|y_{0}\right|^{2}+\left|z_{1}\right|^{2}+\left|z_{2}\right|^{2}=\zeta
$$

Varying the $D$-term leads to two possible partial resolutions ("unresolved" and "fully resolved") as illustrated in Figure 6.
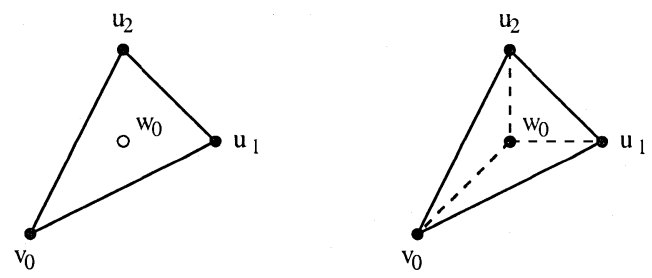

Figure 6. Partial resolutions of the complex cone over $\mathbb{C P}^{2}$.

The "most efficient" description omits the vertex $w_{0}$ as illustrated in the left side of Figure 6. Notice that if we gauge fix to eliminate the corresponding homogeneous coordinate $y_{0}$, there is a residual $\mathbb{Z}_{3}$ gauge symmetry. That is, the complex cone over $\mathbb{C P}^{2}$ is a $\mathbb{Z}_{3}$ orbifold.

In the case of the complex cone over $\mathbb{F}_{0}$, the singularity can be described by means of homogeneous coordinates $y_{0}, y_{1}, y_{2}, z_{1}, z_{2}$ with $U(1)^{2}$ action

$$
\left(\begin{array}{ccccc}
y_{0} & y_{1} & y_{2} & z_{1} & z_{2} \\
-2 & 1 & 0 & 1 & 0 \\
-2 & 0 & 1 & 0 & 1
\end{array}\right)
$$


The moment map with arbitrary $D$-terms $\zeta_{1}, \zeta_{2}$ is given by

$$
\begin{aligned}
& -2\left|y_{0}\right|^{2}+\left|y_{1}\right|^{2}+\left|z_{1}\right|^{2}=\zeta_{1} \\
& -2\left|y_{0}\right|^{2}+\left|y_{2}\right|^{2}+\left|z_{2}\right|^{2}=\zeta_{2} .
\end{aligned}
$$

Notice that the difference between these equations is

$$
\left|y_{1}\right|^{2}-\left|y_{2}\right|^{2}+\left|z_{1}\right|^{2}-\left|z_{2}\right|^{2}=\zeta_{1}-\zeta_{2} .
$$

Varying the $D$-terms leads to four possible partial resolutions (including "unresolved" and "fully resolved") as illustrated in Figure 7. Each of the two partially resolved cases has a curve of $\mathbb{Z}_{2}$ singularities.

The "most efficient" description omits the vertex $w_{0}$ as in the left portions of Figure 7. If we gauge fix the second $U(1)$ in (4.16) to eliminate the homogeneous coordinate $y_{0}$, there is a residual $\mathbb{Z}_{2}$ group action on a conifold point (recognizable from the remaining moment map (4.18)). That is, the complex cone over $\mathbb{F}_{0}$ is a $\mathbb{Z}_{2}$ orbifold of a conifold.
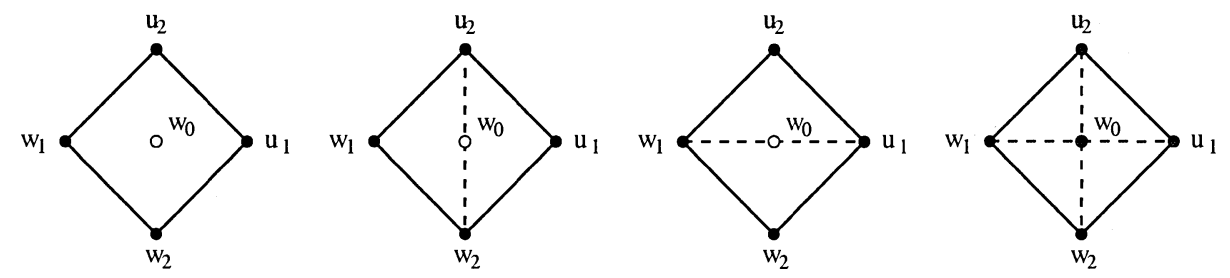

Figure 7. Partial resolutions of the complex cone over $\mathbb{F}_{0}$.

\subsection{Horizon manifolds}

For each of the toric singularities discussed in the previous section, we will now study the corresponding horizon $H$ in some detail, determining its isometry group, its homology, and the parameter space for minimum volume cycles in certain homology classes. (All of these properties will be useful when we subsequently compare $A d S$ compactifications to field theory models.) As we learned in section 2 , when the singularity leads to an $\mathcal{N}=1$ theory the horizon should have an Einstein-Sasaki structure with an associated $U(1)$ action (the $R$-symmetry in the field theory) so that $H / U(1)$ has the structure of a Kähler-Einstein orbifold. The horizon can then be reconstructed as the unit circle bundle in a certain holomorphic line bundle $\mathcal{L}$ over the Kähler-Einstein space.

When the $U(1)$ action is regular, $H / U(1)$ is a Kähler-Einstein manifold (in fact, a del Pezzo surface) and we can use the Leray spectral sequence ${ }^{20}$ for the fibration $H \rightarrow H / U(1)$ to calculate the cohomology and homology of

\footnotetext{
${ }^{20}$ For an introduction to spectral sequences, see [100].
} 
$H$. The del Pezzo surface $H / U(1)$ has vanishing first and third cohomology groups, and its second cohomology group is isomorphic to $\mathbb{Z}^{b}$ for some $b$, the second Betti number of $H / U(1)$. The first Chern class of $\mathcal{L}$ determines a map $\lambda: \mathbb{Z} \rightarrow \mathbb{Z}^{b}$, and a dual map $\lambda^{*}: \mathbb{Z}^{b} \rightarrow \mathbb{Z}$, which appear in the spectral sequence. The nonzero entries in the $E_{2}$ term of the Leray spectral sequence are then:

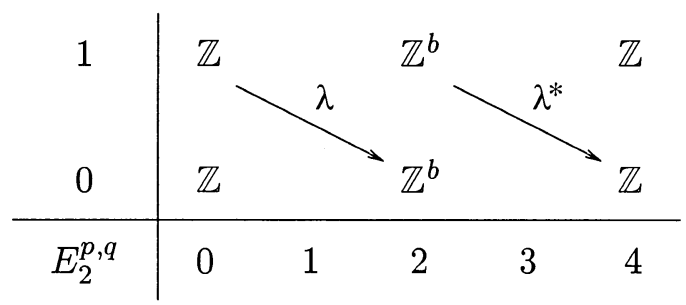

Suppose that $c_{1}(\mathcal{L})$ is divisible by $m$ in $H^{2}(H / U(1))$, so that the cokernel of $\lambda$ contains the $m$-torsion group $\mathbb{Z}_{m}$. Then the $E_{3}=E_{\infty}$ term of the Leray spectral sequence is

\begin{tabular}{c|ccccc}
1 & 0 & 0 & $\mathbb{Z}^{b-1}$ & 0 & $\mathbb{Z}$ \\
0 & $\mathbb{Z}$ & 0 & $\mathbb{Z}^{b-1} \oplus \mathbb{Z}_{m}$ & 0 & $\mathbb{Z}_{m}$ \\
\hline$E_{\infty}^{p, q}$ & 0 & 1 & 2 & 3 & 4
\end{tabular}

We can read off the cohomology of $H$ from the $E_{\infty}$ term as $H^{i}(H)=$ $\bigoplus_{p+q} E_{\infty}^{p, q}$, and determine the homology by Poincaré duality or the universal coefficient theorem. The result is shown in Table 5 .

\begin{tabular}{|c|cccccc|}
\hline$i$ & 0 & 1 & 2 & 3 & 4 & 5 \\
\hline$H^{i}(H)$ & $\mathbb{Z}$ & 0 & $\mathbb{Z}^{b-1} \oplus \mathbb{Z}_{m}$ & $\mathbb{Z}^{b-1}$ & $\mathbb{Z}_{m}$ & $\mathbb{Z}$ \\
\hline$H_{i}(H)$ & $\mathbb{Z}$ & $\mathbb{Z}_{m}$ & $\mathbb{Z}^{b-1}$ & $\mathbb{Z}^{b-1} \oplus \mathbb{Z}_{m}$ & 0 & $\mathbb{Z}$ \\
\hline
\end{tabular}

Table 5. Cohomology and homology of horizon manifolds.

To compute the isometry groups of our horizons, we must work with the explicit descriptions of the horizon manifolds. The $\mathcal{N}=1$ supersymmetry in the associated compactification of the IIB string on $\mathrm{AdS}_{5} \times H$ requires a complex Killing spinor on $H$, and the isometry group of $H$ contains a distinguished $U(1)$ which simply shifts the phase of that Killing spinor-this becomes the $U(1) R$-symmetry in the associated conformal field theory. To determine the symmetries of the supersymmetric theory, we need to compute 
the group of all isometries of $H$ which commute with this $U(1)$. We will call this the group of supersymmetric isometries. ${ }^{21}$

\section{Orbifolds}

For orbifold singularities $\mathbb{C}^{3} / \Gamma$, with $\Gamma \subset S U(3)$, we consider the induced action of $\Gamma$ on $\mathbb{C P}^{2}$. The space $\mathbb{C P}^{2} / \Gamma$ will inherit the structure of a KählerEinstein orbifold, from the Kähler-Einstein metric on $\mathbb{C P}^{2}$. Similarly, the horizon $S^{5}$ of the origin in $\mathbb{C}^{3}$ inherits an action of $\Gamma$, and $S^{5} / \Gamma$ will be the corresponding horizon for $\mathbb{C}^{3} / \Gamma$. In the $\mathcal{N}=1$ case, the $U(1)$ action descends to $S^{5} / \Gamma$ in such a way that $\left(S^{5} / \Gamma\right) / U(1)=\mathbb{C P}^{2} / \Gamma$. (In other words, $U(1)$ acts with charge 1 on each of the three complex variables in $\mathbb{C}^{3}$.) The horizon will be singular if the action of $\Gamma$ on $\mathbb{C}^{3}$ has a non-isolated fixed point locus. When present, the singularities occur along $S^{1}$ s (i.e., in real codimension four within $H^{5}$ ).

The isometries of $S^{5} / \Gamma$ lift to isometries of $S^{5}$ and so lie in $S O(6)$. The commutator in $S O(6)$ of the $U(1) R$-symmetry group is $U(3)$, so in the $\mathcal{N}=1$ case the supersymmetric isometries of $S^{5} / \Gamma$ are given by

$$
\{g \in U(3) \mid g \Gamma=\Gamma g\} / \Gamma \text {. }
$$

For example, when $\Gamma=\mathbb{Z}_{3}$ acting as the center of $S U(3)$, then (4.19) becomes $U(3) / \mathbb{Z}_{3}$, whereas when $\Gamma=\mathbb{Z}_{2} \times \mathbb{Z}_{2}$ acting with generators $(x, y, z) \rightarrow$ $(-x,-y,-z)$ and $(x, y, z) \rightarrow(-x, y,-z)$ then (4.19)becomes $U(1)^{3} \rtimes S_{3}$.

The $U(1)$ action on $S^{5} / \Gamma$ is regular only in the case $\Gamma=\mathbb{Z}_{3}$ [49]. As remarked earlier, this is the horizon of the complex cone over $\mathbb{C P}^{2}$. In this case, the second Betti number $b$ of $H / U(1)=\mathbb{C P}^{2}$ is 1 , and the torsion in the cokernel of $\lambda$ has order $m=3$. The nonzero homology groups are $H_{0}=H_{5}=\mathbb{Z}$ and $H_{1}=H_{3}=\mathbb{Z}_{3}$.

More generally, when $\mathbb{C}^{3} / \Gamma$ has an isolated singularity so that $H=S^{5} / \Gamma$ is a manifold, the homology and cohomology of $H$ can be computed directly with the results given in Table 6 . The computations proceeds as follows: first, the free part of homology and cohomology is concentrated in degrees zero and five, since all $p$-forms can be pulled back to $S^{5}$. Next, since $\pi_{1}(H)=$ $\Gamma$, the first homology group $H_{1}(H)$ is the Abelianization $\Gamma /[\Gamma, \Gamma]$ of $\Gamma$, which we denote by $\Gamma^{\prime}$. Poincaré duality and the universal coefficient theorem then determine $H^{2}, H_{3}$ and $H^{4}$. Finally, by the Hurewicz theorem, $H_{2}(H)=0$, and it follows by Poincaré duality or the universal coefficient theorem that $H^{3}(H)=0$ as well.

\footnotetext{
${ }^{21}$ Similar definitions could be made for horizons corresponding to theories with extended supersymmetry, using the larger $R$-symmetry groups relevant in such cases.
} 


\begin{tabular}{|c|cccccc|}
\hline$i$ & 0 & 1 & 2 & 3 & 4 & 5 \\
\hline$H^{i}\left(S^{5} / \Gamma\right)$ & $\mathbb{Z}$ & 0 & $\Gamma^{\prime}$ & 0 & $\Gamma^{\prime}$ & $\mathbb{Z}$ \\
\hline$H_{i}\left(S^{5} / \Gamma\right)$ & $\mathbb{Z}$ & $\Gamma^{\prime}$ & 0 & $\Gamma^{\prime}$ & 0 & $\mathbb{Z}$ \\
\hline
\end{tabular}

Table 6. Cohomology and homology of manifolds $S^{5} / \Gamma$.

In the case that $\Gamma=\mathbb{Z}_{k}$ is a cyclic group acting diagonally on the coordinates $(x, y, z)$, there are natural three-cycles which represent nontrivial classes in $H_{3}$ given by $x=0, y=0$, and $z=0$. Each is the image of some $S^{3}$ from $S^{5}$, itself modded out by $\Gamma$. Each of these is a minimum volume representative of its homology class. (The precise classes which are represented depend on the action of $\mathbb{Z}_{k}$.)

\section{Conifold}

Turning next to the conifold, the space $H / U(1)$ is $\mathbb{F}_{0}=\mathbb{C P}^{1} \times \mathbb{C P}^{1}$, as already noted in section 2 . The Kähler-Einstein metric on this space is compatible with a toric construction of the space; the simplest toric construction uses homogeneous coordinates $x_{0}, x_{1}, y_{0}, y_{1}$ with a $U(1)^{2}$ action given by the charge matrix

$$
\left(\begin{array}{cccc}
x_{0} & x_{1} & y_{0} & y_{1} \\
1 & 1 & 0 & 0 \\
0 & 0 & 1 & 1
\end{array}\right) .
$$

To have equal-volume round metrics on the two-spheres, we must insist that the two $D$-terms for the two $U(1)$ 's be equal.

Line bundles over this space can be be described in toric geometry by including a fifth homogeneous variable $p$. The line bundle relevant to the conifold has toric data

$$
\left(\begin{array}{ccccc}
x_{0} & x_{1} & y_{0} & y_{1} & p \\
1 & 1 & 0 & 0 & -1 \\
0 & 0 & 1 & 1 & -1
\end{array}\right) .
$$

This time we use $D$-terms of zero, in order to get a cone-type toric variety. In order to more easily recognize this singularity, we can use a modified basis for the $U(1)$ 's, with charge matrix

$$
\left(\begin{array}{ccccc}
x_{0} & x_{1} & y_{0} & y_{1} & p \\
1 & 1 & -1 & -1 & 0 \\
0 & 0 & 1 & 1 & -1
\end{array}\right) .
$$


The horizon will be the unit circle bundle inside the line bundle, defined by $|p|^{2}=1$. Thus, using this condition, we can describe the horizon by two $D$-term equations

$$
\left|x_{0}\right|^{2}+\left|x_{1}\right|^{2}-1=0, \quad\left|y_{0}\right|^{2}+\left|y_{1}\right|^{2}-1=0,
$$

and mod out by two $U(1)$ 's. One of the $U(1)$ 's can be used to gauge-fix $p$, setting it equal to 1 . This leaves another $U(1)$, acting on the remaining variables as

$$
\left(x_{0}, x_{1}, y_{0}, y_{1}\right) \mapsto\left(e^{i \theta} x_{0}, e^{i \theta} x_{1}, e^{-i \theta} y_{0}, e^{-i \theta} y_{1}\right) .
$$

Thus we see a description of the horizon of the conifold in the form $\left(S^{3} \times\right.$ $\left.S^{3}\right) / U(1)$ with action (4.24).

The $U(1) R$-symmetry acts by rotating the phase of $p$ in the original description; after our gauge fixing, and with an appropriate choice of gauge for the remaining $U(1)$, it acts with charge 1 on the complex variables $x_{0}$, $x_{1}, y_{0}, y_{1}$. The commutator of this $U(1)_{R}$ in $\operatorname{Isom}\left(S^{3} \times S^{3}\right)$ is $U(2)^{2} \rtimes \mathbb{Z}_{2}$ (with the $\mathbb{Z}_{2}$ acting by exchanging the factors). Since the $U(1)$ given by (4.24) is a normal subgroup of $U(2)^{2} \rtimes \mathbb{Z}_{2}$, we find that the supersymmetric isometries of the conifold horizon are given by

$$
\left(U(2)^{2} \rtimes \mathbb{Z}_{2}\right) / U(1),
$$

with the $U(1)$ action given by (4.24).

The homology in this case is determined by the second Betti number $b=2$ of $H / U(1)=\mathbb{C P}^{1} \times \mathbb{C P}^{1}$, and the fact that the cokernel of $\lambda$ is torsionfree (i.e., $m=1$ ). Thus, the nonzero homology groups are $H_{0}=H_{2}=H_{3}=$ $H_{5}=\mathbb{Z}$. (In fact, $H$ is topologically the product of $S^{2}$ and $S^{3}[93]$.)

Once again we can describe minimum-volume representatives for certain generators of $H_{3}(H)$. Representing $H$ as $\left(S^{3} \times S^{3}\right) / U(1)$, there are two natural projections to $S^{3} / U(1)=S^{2}$ (the left factor and the right factor). Each gives a description of $H$ as being swept out by an $S^{2}$ of minimumvolume $S^{3}$ 's (of homology class $A_{i}$ ). However, the two families of $S^{3}$ 's belong to opposite homology classes, i.e., $A_{1}+A_{2}=0$ in homology.

\section{Complex cone over $\mathbb{F}_{0}$}

The case of the complex cone over $\mathbb{F}_{0}$ has many similar aspects to the conifold. This time we use the anti-canonical bundle of $\mathbb{F}_{0}$ as $\mathcal{L}$, whose toric data is

$$
\left(\begin{array}{ccccc}
x_{0} & x_{1} & y_{0} & y_{1} & p \\
1 & 1 & 0 & 0 & -2 \\
0 & 0 & 1 & 1 & -2
\end{array}\right) .
$$


The unit circle bundle is $|p|^{2}=1$, which modifies the $D$-term equations to

$$
\left|x_{0}\right|^{2}+\left|x_{1}\right|^{2}-1=0, \quad\left|y_{0}\right|^{2}+\left|y_{1}\right|^{2}-1=0,
$$

as before. This time, however, when we use one of the $U(1)$ 's to gauge-fix $p$, there is a residual $\mathbb{Z}_{2}$ symmetry. Thus, in addition to modding out $S^{3} \times S^{3}$ by $U(1)$ as specified in 4.24 , we need to $\bmod$ out by an additional $\mathbb{Z}_{2}$, acting as

$$
\left(x_{0}, x_{1}, y_{0}, y_{1}\right) \mapsto\left(-x_{0},-x_{1}, y_{0}, y_{1}\right) .
$$

The horizon is thus $\left(S^{3} \times S^{3}\right) /\left(U(1) \times \mathbb{Z}_{2}\right)$.

Since $U(1) \times \mathbb{Z}_{2}$ is normal in $U(2)^{2} \rtimes \mathbb{Z}_{2}$, the group of supersymmetric isometries is

$$
\left(U(2)^{2} \rtimes \mathbb{Z}_{2}\right) /\left(U(1) \times \mathbb{Z}_{2}\right),
$$

with the $U(1) \times \mathbb{Z}_{2}$ action given by (4.24) and (4.28).

The homology in this case is again determined by the second Betti number $b=2$, and the torsion $\mathbb{Z}_{2}$ in the cokernel of $\lambda$ (i.e., $m=2$ ). Thus, the nonzero homology groups are $H_{0}=H_{2}=H_{5}=\mathbb{Z}, H_{1}=\mathbb{Z}_{2}$, and $H_{3}=\mathbb{Z} \oplus \mathbb{Z}_{2}$. This horizon $H$ is topologically the quotient of $S^{2} \times S^{3}$ by a freely acting $\mathbb{Z}_{2}$. (So in particular, $\pi_{1}(H)=\mathbb{Z}_{2}$.)

Again, there are minimum-volume representatives for certain classes in $H_{3}$, consisting of two families of three-spheres, such that their sum is the torsion element (i.e., $A_{1}+A_{2}$ represents the two-torsion in $H_{3}$ ). The parameter space for each of these families is a two-sphere.

\section{Suspended pinch point}

Finally, in the case of the suspended pinch point, we can again make a similar construction. We begin with a product of two copies of $\mathbb{W} \mathbb{C P}^{1,2}$, whose toric description is

$$
\left(\begin{array}{cccc}
x_{0} & x_{1} & y_{0} & y_{1} \\
1 & 2 & 0 & 0 \\
0 & 0 & 1 & 2
\end{array}\right) .
$$

(As before, we keep the $D$-terms equal.) The line bundle of interest is $1 / 3$ of the anti-canonical bundle, which has a toric description

$$
\left(\begin{array}{ccccc}
x_{0} & x_{1} & y_{0} & y_{1} & p \\
1 & 2 & 0 & 0 & -1 \\
0 & 0 & 1 & 2 & -1
\end{array}\right) .
$$

(using zero for both $D$-terms). In another basis for the $U(1)$ 's, we find

$$
\left(\begin{array}{ccccc}
x_{0} & x_{1} & y_{0} & y_{1} & p \\
1 & 2 & -1 & -2 & 0 \\
0 & 0 & 1 & 2 & -1
\end{array}\right) .
$$


In this form, we easily recognize the toric data for the suspended pinch point.

To find the horizon, we again impose $|p|^{2}=1$ and find equations

$$
\left|x_{0}\right|^{2}+2\left|x_{1}\right|^{2}-1=0, \quad\left|y_{0}\right|^{2}+2\left|y_{1}\right|^{2}-1=0 .
$$

We use one $U(1)$ to gauge fix $p$ to 1 , leaving a second $U(1)$ with action

$$
\left(x_{0}, x_{1}, y_{0}, y_{1}\right) \mapsto\left(e^{i \theta} x_{0}, e^{2 i \theta} x_{1}, e^{-i \theta} y_{0}, e^{-2 i \theta} y_{1}\right) .
$$

This $U(1)$ action is not regular: the orbits with $x_{0}=y_{0}=0$ are half the size of the generic orbits. There is an $S^{1}$ of such orbits on the horizon manifold itself.

So the horizon again has a description as $\left(S^{3} \times S^{3}\right) / U(1)$, but with the $U(1)$-action given by (4.34), which leads to singularities along an $S^{1}$.

To find the group of supersymmetric isometries in this case, note that the normalizer in $U(2)^{2} \rtimes \mathbb{Z}_{2}$ of the $U(1)$ action (4.34) is $U(1)^{4} \rtimes \mathbb{Z}_{2}$; thus, the group of supersymmetric isometries is

$$
\left(U(1)^{4} \rtimes \mathbb{Z}_{2}\right) / U(1)
$$

with the $U(1)$ action given by (4.34).

Since this space has singularities, the usual homology might not be the right thing to calculate. We can, however, still study the parameter spaces for minimum volume representatives of certain kinds of cycles. As in the conifold case, there are two natural projections, in this case projections to $\mathbb{W C P}^{1,2}$, which express $H^{5}$ as a family of three-cycles. However, in this case there is one three-cycle which is distinguished as having smaller volume-the one which lies over the unique orbifold point in $\mathbb{W C P}^{1,2}$. We thus use those two three-cycles as minimum-volume representatives. The corresponding classes $A_{i}$ satisfy $A_{1}+A_{2}=0$ in the appropriate homology theory.

\section{An orbifold model}

We have conjectured that for each of the singularities discussed in the previous section, and for each $N$, there should be a dual conformal field theory (thought of as living on the boundary of $A d S_{5}$ ). In order to test this conjecture, we need to be able to identify the low-energy $C F T$ on the worldvolume, and perform computations there as well as in the compactification on $A d S_{5} \times H^{5}$. Our approach to the former problem is based on the construction [101] of the theory of branes at orbifolds as an appropriate projection of the theory of branes on the covering space. This method was used in [102] to compute the worldvolume theory for branes at an orbifold singularity when the transverse space is locally described as $\mathbb{C}^{3} / \Gamma$. One of the useful 
products of this analysis, applied for example in [103, 104], is that it yields a map between resolutions of the singularity and deformations of the field theory. Using this we can find the field-theoretic description of the partial resolutions of a given quotient singularity. As discussed in section 4.2, by studying the case $\Gamma=\mathbb{Z}_{m} \times \mathbb{Z}_{m}$ this method in principle allows us to treat all toric Gorenstein canonical singularities. Singularities of this type were studied from a different point of view in [25] . In this work we will focus on the simplest such model, the case $\Gamma=\mathbb{Z}_{2} \times \mathbb{Z}_{2}$ and its partial resolutions. We will also discuss the complex cones over $\mathbb{C P}^{2}$ and $\mathbb{F}_{0}$.

The AdS/CFT correspondence for D3-branes at quotient singularities in three complex dimensions was studied in [12, 14, 15, 18, 19, 20, 23]. From the point of view of the $A d S$ compactification, the quotient acts on the $S^{5}$, leaving the $A d S$ space unchanged. Thus we find in these examples horizons of the form $H^{5}=S^{5} / \Gamma$ as discussed in section 4.3 above. On the brane worldvolume, the prescription of [102] leads for $\Gamma \subset S U(3)$ to an $\mathcal{N}=1$ model, with a gauge group which is a product of $U(N)$ factors and chiral matter in bifundamental representations. The one-loop $\beta$-functions for the $S U(N)$ couplings in these theories vanish $[14,15,20]$. In the large $N$ limit, these theories are in fact finite, as shown in the latter two references. Before launching into the computation for the $\mathbb{Z}_{2} \times \mathbb{Z}_{2}$ model, we make some general remarks regarding orbifold models. The goal here is to set out our understanding of these systematically with a view towards the generalizations we will attempt in section 6 . Since the examples we discuss here have been studied in detail in the references given, we will be brief in presenting them and focus on those aspects which are most relevant to our generalizations.

\subsection{Orbifolds and the $A d S / C F T$ correspondence}

For simplicity we initially frame our discussion for a cyclic group $\Gamma=\mathbb{Z}_{k}$ acting on three complex coordinates as $X^{I} \rightarrow \omega^{a_{I}} X^{I}$ with $\omega$ a $k^{\text {th }}$ root of unity. The worldvolume theory will be supersymmetric if $\Gamma \subset S U(3)$, i.e. $\sum_{I} a_{I}=0$. The extension to other groups is not difficult. The essential idea behind the construction of [101] is that the theory of $N$ branes near the singularity can be constructed by a projection from the theory of $N k$ branes on the covering space $\mathbb{C}^{3}$. The resulting worldvolume theory is then found to be an $\mathcal{N}=1$ gauge theory with gauge group $U(N)^{k}$ and chiral multiplets $X_{l, l+a_{I}}^{I}$ in bifundamental representations $(\mathbf{N}, \overline{\mathbf{N}})$ under $U(N)_{l} \times U(N)_{l+a_{I}}$ where $l=1, \ldots k$. These interact via a cubic superpotential that is simply the restriction to these modes of the cubic superpotential of the original $\mathcal{N}=4$ theory. Note that there are $3 N$ fundamental and anti-fundamental chiral multiplets under each $S U(N)$ factor, so that the one-loop $\beta$-functions vanish. Also, since all matter is in bifundamental representations, the diagonal $U(1)$ subgroup decouples completely from the theory and describes a free photon. 
As discussed in $[5,7,105]$ this photon is absent from the $A d S$ description. We will ignore this issue in the sequel, but note that in our theories there remain $k-1$ Abelian factors in the gauge group, under which some of the massless matter is charged. This means of course that these decouple in the infrared and have no interesting dynamics in the conformal limit. In spite of this decoupling, the fate of these gauge symmetries is still of interest and will be discussed further below.

The closed-string sector of a IIB compactification on a quotient space (or, in the low-energy limit, on a space with a quotient singularity) bears some distinctive marks of its orbifold character. Notable among these are the existence of twisted sectors and a "quantum" symmetry which acts on states in these sectors. Twisted-sector states describe strings which, in $\mathbb{C}^{3}$, close only up to the action of an element $g \in \Gamma$ (which labels the sector). The quantum symmetry can be summarized geometrically as the statement that the identity of $g$ is conserved by interactions (where for multi-string states the group elements should be multiplied to obtain the total "twist"). Massless states in this sector arise from strings localized near loci fixed by g. In Calabi-Yau compactifications, the massless states in twisted sectors of an orbifold singularity are known to correspond to the moduli of the singularity. Namely, for each two-cycle in a resolution of the singularity we have a massless $(\mathcal{N}=2)$ hypermultiplet. The four real scalars are given by the period of the Kähler class about the two-cycle; the periods of the two two-form fields $b_{N S}$ and $b_{R R}$; and the period of the four-form field about the dual four-cycle. In terms of the $S U(2)_{R}$ symmetry these form a complex doublet. There are additional light states in four dimensions associated to the degenerating two-spheres. D3-branes wrapping these two-spheres describe strings in the four-dimensional theory. These are BPS states, and the string tension vanishes when the two-sphere degenerates to zero volume and the corresponding two-form periods vanish as well.

When the supersymmetry is broken to $\mathcal{N}=1$ by the presence of the transverse D3-branes (or in the $A d S$ picture by the curvature and five-form flux) the hypermultiplet of twisted-sector moduli breaks up into two chiral multiplets with, in principle, different masses. The two two-form periods pair up to form the complex scalar $B=b_{R R}+i b_{N S}$ in one chiral multiplet $B$, and the Kähler mode $r$ is complexified by the four-form period $a$ to form the complex scalar $R=a+i r$ in a second chiral multiplet $R$. The couplings of this latter multiplet are perhaps most naturally described if we dualize the real part; the result is a linear chiral multiplet, containing a real scalar $r$ and a two-form potential $c$ (the integral of the self-dual RR four-form over the two-cycle). 


\section{Non-isolated singularities}

The discussion we wish to make will depend upon the nature of the quotient singularity. In particular, quotient singularities can occur in codimension two (along complex curves) or three (at points, the complement of the singular point being smooth). We begin with the case of a non-isolated singularity. As an example for this case, we consider the $\mathbb{Z}_{k}$ action given by $\vec{a}=(1,-1,0)$. The structure transverse to the fixed $Z$ axis is then $\mathbb{C}^{2} / \mathbb{Z}_{k}$. Resolving this singularity leads to $k-1$ homology two-cycles $\Sigma_{i}$ with an intersection matrix given by (minus) the Cartan matrix of $A_{k-1}$. In the scaling limit, this quotient leads to an $\mathcal{N}=2$ theory with gauge group $U(N)^{k}$ (combining the vector multiplets of $\mathcal{N}=1$ with the projections of $Z$ ) and bifundamental hypermultiplets transforming in the $(\mathbf{N}, \overline{\mathbf{N}})$ representation of $U(N)_{l} \times U(N)_{l+1}[101]$. The quantum $\mathbb{Z}_{k}$ symmetry acts in these models by cyclic permutations of the $k$ factors of the gauge group, and corresponding permutations of the charged hypermultiplets. In general, the theory also has an $S U(2) \times U(1) R$-symmetry.

The four-dimensional theory in this case can be obtained by dimensional reduction of a six-dimensional theory, and the spectrum and couplings of the twisted sectors were computed in [101] . The couplings of twisted RR fields are computed by an explicit worldsheet calculation following [106]. The couplings of the other fields then follow by supersymmetry. These calculations show that the scalar $a_{j}$ arising from the RR four-form in the $j$ th twisted sector has nontrivial gauge transformation properties under the Abelian factors in the gauge group

$$
a_{j} \rightarrow a_{j}+\sum_{l=1}^{k} \omega^{j l} \epsilon_{l} .
$$

Thus the $U(1)$ factors in the gauge group are broken, and the low-energy gauge group is $S U(N)^{k}$. The supersymmetric completion of the coupling implied by (5.1) shows that the metric modes $r_{j}$ parameterize the FI $D$ terms. Note that the Abelian factors in the gauge group (except for the trivial diagonal) are still present as global symmetries in the worldvolume field theory. We refer to the conserved charges as baryon numbers under the various $S U(N)$ factors. Thus the global symmetry group in general contains $U(1)^{k-1}$ in addition to the $R$-symmetry.

The chiral primary operators charged under these symmetries are formed by antisymmetrizing the product of $N$ bifundamental fields on both gauge indices. These would actually be absent if the gauge symmetry included the Abelian factors, so their existence verifies the arguments above that the latter are broken. These baryons, unlike the baryon vertex studied in [107], represent actual particles in the theory. Identifying the spectrum of baryons and identifying these states in the dual $A d S$ model provides strong evidence for the conjecture; in particular, because these states become infinitely mas- 
sive (in $A d S$ ) or have divergent conformal weights (in the CFT) in the large $N$ limit, this comparison suggests that some aspects of the theories agree at finite $N$. In the model at hand, each of the bifundamental hypermultiplets leads according to this prescription to a hypermultiplet of baryon states. The quantum symmetry permutes these baryons as it does the bifundamental fields, and we can construct a basis of linear combinations with charges corresponding to the twisted sectors by discrete Fourier transform. Thus, there is one baryon (and one antibaryon) state in each sector, with conformal weight $N$ (which is determined by the $R$-charge, and is thus protected from quantum corrections).

The supersymmetric completion of the RR two-form coupling shows that the two-form periods appear in the worldvolume action as

$$
\frac{1}{8 \pi} \operatorname{Im} \int d^{2} \theta \sum_{j, l} \omega^{j l} B_{j} \operatorname{tr}\left(W_{l}^{2}\right)
$$

Thus, the two-form periods enter the worldvolume theory as gauge couplings and $\theta$ angles. It is interesting in this context that the two-form periods naturally parameterize a two-torus, since $b_{N S} \sim b_{N S}+1$ while $b_{R R} \sim b_{R R}+$ $\tau_{S}$ where $\tau_{S}=\frac{\chi}{2 \pi}+\frac{2 \pi i}{g}$ is the complexified string coupling. A striking confirmation of this identification was found in [14] , where this periodicity was shown to correspond to the known $S$-duality group for an $\mathcal{N}=2$ orbifold model. The (bare) gauge couplings are thus given by $\tau_{l}=\tau_{S}+\sum_{j} \omega^{j l} b_{j}$. The point $b_{j}=0$, which we interpret as the undeformed quotient theory, will have all two-form periods equal to one-half their maximal value [108] . We define our variables so that this point corresponds to $b_{j}=0$. Note that the nonzero two-form periods give the wrapped D3-brane strings mentioned above a tension of order $\alpha^{\prime}$, so they are expected to be absent from the low-energy theory. Indeed, the model we have described has no candidate tensionless strings, consistent with our interpretation of this point as the orbifold point at which the CFT is nonsingular.

From the point of view of the dual $A d S$ compactification, the horizon $H^{5}=S^{5} / \Gamma$ is singular in these cases, as explained in section 4.3. The singularity occurs along the image of the circle $|Z|=1$, itself a circle in the quotient (of $1 / k$ the size), with the transverse space described locally by $\mathbb{C}^{2} / \mathbb{Z}_{k}$. A supergravity theory compactified on this space would be singular, but as first pointed out in [12] we expect the IIB theory to be well-behaved. In particular, we expect the same general structure of twisted sectors and twisted moduli for the compactification. The spectrum of states in the untwisted sector can be found by projecting the known spectrum of the $A d S_{5} \times S^{5}$ compactification to $\Gamma$-invariant states. Restricting attention to the KaluzaKlein states of the supergravity theory reproduces the spectrum of chiral primary operators in the untwisted sector of the worldvolume theory whose conformal dimensions remain finite in the large $N$ limit [18] . (In the field 
theory, untwisted states can be easily recognized by their invariance under the global "quantum" symmetry). The twisted sector states were first studied in detail by Gukov [23] . The background curvature and five-form flux change the spectrum in a calculable way, and applying this correction Gukov was able to reproduce the spectrum of chiral primary fields charged under the quantum symmetry from the spectrum of twisted states in the $A d S$ compactification in $\mathcal{N}=2$ orbifolds. In particular, this analysis demonstrates that the quantum symmetry is realized in these models as the quantum symmetry associated to the quotient singularity in $H^{5}$.

The work of $[18,23]$ did not include the baryons in the spectra of the models. (These were, however, subsequently discussed in [32] .) Along the lines described by Witten [107], one expects that the baryons are represented by D3-branes wrapped around three-cycles in $H^{5}$. Witten considers related theories, and points out that a D3-brane wrapping a nontrivial three-cycle in $H^{5}$ would lead to a BPS particle with mass $m \sim V_{3} / g$. Since $V_{3} \sim R^{3}$ with $R \sim(g N)^{1 / 4}$, the conformal dimension of the operator in the CFT coupling to this field is

$$
\Delta \sim m R \sim N
$$

independent of $g$. This is in accordance with the baryon-like particles we predict.

Thus, we would like to find that the horizon in this case has a homology group $H_{3} \supseteq \mathbb{Z}^{k-1}$. Since the horizon is singular, this is somewhat delicate to compute explicitly and we give a heuristic description, motivated by the fact that it yields physically reasonable answers. As noted above, the local structure transverse to the singular circle is $\mathbb{C}^{2} / \mathbb{Z}_{k}$. This singularity, when resolved, leads to a chain of exceptional $\mathbb{C P}^{1}, \mathrm{~s} \Sigma_{i}$ with an intersection matrix given by (minus) the Cartan matrix of $A_{k-1}$. We can construct a basis of three-cycles dual to this, given by $C_{j}=\Sigma_{j}^{*} \times S^{1}$. One can show that these transform under the quantum $\mathbb{Z}_{k}$ symmetry in all nontrivial one-dimensional representations, in accord with our expectation that we find one baryonic state in each twisted sector (the antibaryons are of course represented by D3-branes wrapped with the opposite orientation). In the quotient space, of course, all of these have vanishing volume and hence the scaling argument above is suspect. However, at the orbifold point we have nonzero two-form periods about these cycles, and we expect this to lead to a nonzero mass for these particles, scaling by dimensional analysis as in (5.3) . Constructing the untwisted baryon state is more difficult, since the supergravity approximation breaks down for states as massive as this.

These same three-cycles lead in the $A d S$ theory to additional gauge symmetries, whose gauge fields $A_{j}=\int_{C_{j}} A^{(4)}$ are the reduction of the selfdual RR four-form on the three-cycles. The baryon states described above are charged under these symmetries, which hence should correspond to the 
baryon number global symmetries in the $C F T$. The charges of the baryons suggest that $A_{j}$ couples to $\sum_{l} \omega^{j l} J_{l}$ where $J_{l}$ is the worldvolume current for baryon number under the $l^{\text {th }}$ factor in the gauge group. In section 4 we saw that the $R$-symmetry of the $C F T$ is realized as a gauge symmetry in the $A d S$ compactification related to isometries of $H^{5}$. In general, the Abelian symmetry described here will complete the continuous global symmetries of the worldvolume theory, so that we have a complete correspondence. In particular cases there may be an enhanced global symmetry on the worldvolume. As we will see in the examples we treat in detail, this will be matched by enhanced isometry groups for the horizon.

The discrete global symmetries of the worldvolume theory will in general include the quantum symmetry mentioned above. This will not act on the geometric horizon $H^{5}$, as we might expect for quantum symmetries. On the other hand, there will be an action on the cycles $C_{j}$ and hence on the baryon spectrum, as discussed above. There is one additional discrete symmetry that will arise in all of these models. In the field theory this is implemented as charge conjugation, exchanging the two chiral multiplets in a hypermultiplet. The interpretation of this in the $A d S$ model was discussed in [30] . Charge conjugation reverses the orientation of the open strings, and so corresponds to the action of the center of the $S L(2, \mathbb{Z})$ duality group of the IIB theory. This acts on the two-form fields as $b_{N S} \rightarrow-b_{N S}$ and $b_{R R} \rightarrow-b_{R R}$. It thus reverses the sign of the two-form periods. This symmetry is thus unbroken for $B=0$, but because of the periodicity, also for the orbifold value $B=$ $\frac{1}{2}\left(1+\tau_{S}\right)$, which describes the origin of our deformation space, as described above.

\section{Isolated singularities}

We now turn to the case of isolated quotient singularities occurring in codimension three. We consider the quotient $\mathbb{C}^{3} / \mathbb{Z}_{k}$ with $a_{I}$ all nonzero. (This is isolated for most choices of the $a_{I}$ when $k$ is odd, which we assume). Resolving this singularity leads to $(k-1) / 2$ new homology two-cycles. A new feature is that the dual four-cycles are in this case localized near the singularity. The $k-1$ twisted sector fields are constrained by a reality condition

$$
B_{j}=B_{k-j}^{*} \quad R_{j}=R_{k-j}^{*} .
$$

The construction of [102] leads here to an $\mathcal{N}=1$ theory with gauge group $U(N)^{k}$, bifundamental chiral multiplets $X_{l, l+a_{I}}^{I}$ and a cubic superpotential (the restriction to the surviving fields of the cubic superpotential from $\mathcal{N}=4$ super Yang-Mills theory). As always, the diagonal $U(1)$ decouples and is not included in the dual $A d S$ model. Once more, the quantum $\mathbb{Z}_{k}$ symmetry acts by permuting the factors in the gauge group and permuting the chiral multiplets as dictated by their representation content. In addition, the model 
has generically a $U(1)^{3}$ global symmetry, ${ }^{22}$ the $I^{\text {th }}$ factor of which acts by phases on all the fields descended from $X^{I}$. One combination of these is anomalous and can be combined with the naïve $U(1)_{R}$ symmetry to form a non-anomalous $U(1)_{R}^{\prime}$.

An important observation [109] is that for these singularities the worldvolume theory is chiral and the Abelian factors in the gauge group as written above suffer from anomalies. This can be easily seen in the example above in which the $U(1)$ factors have mixed anomalies with the nonabelian factors. Under Abelian transformations with parameters $\epsilon_{l}$ the effective action changes by

$$
\sum_{l l^{\prime}} \mathcal{A}_{l l^{\prime}} \epsilon_{l} \operatorname{tr}\left(F_{l^{\prime}} \wedge F_{l^{\prime}}\right)
$$

with

$$
\mathcal{A}_{l l^{\prime}}=N\left(\delta_{l^{\prime}, l+a_{I}}-\delta_{l, l^{\prime}-a_{I}}\right) .
$$

This is puzzling, since we have derived the model in what seems like a consistent manner from an obviously well-defined string theory. The resolution of this puzzle was described in [109] . ${ }^{23}$ It requires an additional coupling (which can be expressed either in terms of the two-form potentials $c_{j}$ or the scalars $a_{j}$ )

$$
\frac{1}{k} \sum_{j=1}^{k-1} e_{j} c_{j} \wedge * \operatorname{tr}\left(\gamma^{-j} I_{G}\right)=\frac{1}{k} \sum_{j=1}^{k-1} e_{j} \sum_{l=1}^{k} \omega^{-j l} a_{j} \operatorname{tr}\left(F_{l} \wedge F_{l}\right)
$$

where $I_{G}$ is the Chern-Simons form for the gauge fields and $e_{j}$ is a constant given by

$$
e_{j}=\prod_{I=1}^{3} 2 \sin \left(\pi j a_{I}\right)
$$

We are not aware of a direct calculation of this coupling along the lines of $[106,101]$ but it easy to verify that it indeed cancels the anomaly $(5.5)$ when we take into account the transformation properties of $a_{j}$. In [101] a version of this argument was used to demonstrate the need for the Chern-Simons couplings. In the $\mathcal{N}=2$ examples above $e_{j}=0 .{ }^{24}$

As in the previous case, the Chern-Simons couplings show that the $U(1)$ factors in the gauge group (apart from the diagonal) are broken, and the $D$-terms are given by the background moduli as [102]

$$
\zeta_{l}=\sum_{j=1}^{k-1} \omega^{j l} R_{j} .
$$

\footnotetext{
${ }^{22}$ The global symmetry group is larger if the $a_{I}$ 's are not all distinct.

${ }^{23}$ The mechanism described here is essentially an extension of the ideas of [110] . These were applied in a similar context to six-dimensional type I compactifications in [111].

${ }^{24}$ In general, for a non-isolated singularity leading to an $\mathcal{N}=1$ theory the coefficient $e_{j}$ corresponding to the subgroup fixing the singular curve will vanish.
} 
These are real by (5.4) and sum to zero since we omit the $j=0$ term in (5.9). Note that in this case the baryon number symmetries are absent as global symmetries as well. From the point of view of the field theory they are broken by anomalies. (Note that the closely related gauge symmetries which act as baryon number on the worldvolume but also act on the RR fields are free of anomalies but, as discussed above, are broken by $\mathrm{RR}$ expectation values.)

Once more, the absence of the Abelian factors predicts the presence of baryons in the spectrum. In general, there will be three families of $k$ baryons arising from the projections of $X^{I}$, forming three $k$-dimensional representations of $\mathbb{Z}_{k}$. We thus predict three baryons in each sector. Since the baryon number symmetries are broken by anomalies, baryon number will not in general be conserved. The three baryonic states in each sector will however be distinguished by their charges under the $U(1)^{3}$ symmetry mentioned above, since the baryon formed as $\left(X^{I}\right)^{N}$ will have charge $N$ under the $I^{\text {th }} U(1)$.

The $A d S$ dual theory is obtained [12] by letting the quotient group act on the horizon. In this case, as described in section 4.3, since the singularity is isolated the horizon is smooth. For the Abelian $\Gamma$ we are discussing here, the supersymmetric isometries given by (4.19) will extend the $R$-symmetry to at least the $U(1)^{3}$ Cartan subgroup of $U(3)$, in agreement with the continuous global symmetry of the generic quotient model as discussed above. In particular cases, this may be enhanced. Note that in this isolated case the self-dual RR four-form gives rise to no gauge fields in the $A d S$ compactification because the homology is pure torsion; this reflects the breaking by anomalies of the corresponding global symmetries in the worldvolume theory. In addition, charge conjugation, together with the permutation $i \rightarrow k-i$ on the gauge groups and the corresponding permutation on the chiral matter multiplets, acts as a $\mathbb{Z}_{2}$ symmetry. In the $A d S$ theory this will as usual be realized as the center of the $S L(2, \mathbb{Z})$ duality group. Once more, this is a symmetry of the orbifold theory with both two-form periods equal to onehalf their maximal value. In these models as well we find no trace of the tensionless strings expected to arise when the two-form periods vanish. This is a somewhat stronger statement here than in the non-isolated case. Since in this case the strings would be constrained by a potential to move on the brane worldvolume we would expect them to represent fluctuating degrees of freedom in the field theory.

The projection to $\Gamma$-invariant states of the supergravity spectrum on $S^{5}$ reproduces the chiral primary untwisted states whose conformal weight remains finite at large $N$ in the worldvolume theory [18] . The origin of the twisted states is not so clear. Because the group action is free, twisted states involve strings with a minimal length of order $R$, so naïvely these cannot correspond to the charged vertex operators of low dimension present 
in the $C F T$. An example of the latter would be

$$
\sum_{l=1}^{k} \omega^{j l} \operatorname{tr}\left(W_{l}^{2}\right),
$$

for $j=1, \ldots k$, with conformal dimension 2 . We do not know a satisfactory resolution to this problem.

We can also consider the $A d S$ counterparts to the baryon states. We would like to describe these as D3-branes wrapped on three-cycles in $H^{5}$; the only available cycles are torsion classes. In fact, as shown in 4.3 the relevant homology group is $H_{3}\left(H^{5}\right)=\mathbb{Z}_{k}$. The $k-1$ torsion cycles have three-volumes of order $R^{3}$ so D3-branes wrapping them will lead to BPS particles with mass as in (5.3). Note that these torsion cycles do not lead to additional gauge symmetry in the $A d S$ compactification, in agreement with the fact that the corresponding global symmetries on the worldvolume are broken by anomalies.

As discussed in 4.3, there are explicit geometric representatives for some of these cycles. Consider an $S^{3} \subset S^{5}$ determined by setting $X^{I}=0$ for some $I$. This (homologically trivial) submanifold is preserved by $\Gamma$, so projects to a submanifold in the quotient, given by $S^{3} / \Gamma$. The triviality upstairs, however, only demonstrates that the cycle so obtained is a torsion cycle. We thus find three representatives (labelled by $I$ ) for each homology class in $H_{3}$, in agreement with the baryon spectrum in the $C F T$. The three states are characterized by their charges under the $U(1)^{3}$ symmetry. But the representation theory of $U(1)^{3} \rtimes \mathbb{Z}_{k}$ together with the fact that the charge under the diagonal $U(1)$ is determined by the five-form flux will fix these to agree with those found in the worldvolume theory. Note that the fact that these are associated to D3-branes wrapping torsion cycles implies that their number is conserved only mod $k$. This is in accord with the fact that baryon number is broken (note that the mod $k$ conservation is not related to an unbroken subgroup of this but rather to the quantum symmetry).

\subsection{Branes at a $\mathbb{Z}_{2} \times \mathbb{Z}_{2}$ singularity}

We now turn to a detailed study of a particular quotient singularity, which we will use as a tool to generate new examples in section 6 . We first discuss the worldvolume theory, comparing in the next subsection with the $A d S$ picture. We consider the theory of $N$ D3-branes at the origin of a space locally described as $\mathbb{C}^{3} / \mathbb{Z}_{2} \times \mathbb{Z}_{2}$. We can take the $\mathbb{Z}_{2} \times \mathbb{Z}_{2}$ action

$$
(X, Y, Z) \rightarrow(-X,-Y, Z)
$$




$$
(X, Y, Z) \rightarrow(-X, Y,-Z)
$$

Following [102] we model $N$ branes near this by using $4 N$ branes on the covering space, leading to an $\mathcal{N}=4$ theory with gauge group $U(4 N)$ on the worldvolume. This is projected to the orbifold by letting the discrete group act on Chan-Paton indices via the regular representation in addition to its action on the spacetime indices. The projection leaves an $\mathcal{N}=1$ theory with gauge group $U(N)^{4}$ (of which the diagonal $U(1)$ decouples completely) and the chiral multiplets surviving the projection are

$$
\begin{aligned}
& X_{14}, X_{23}, X_{41}, X_{32} \\
& Y_{13}, Y_{31}, Y_{24}, Y_{42} \\
& Z_{12}, Z_{21}, Z_{34}, Z_{43}
\end{aligned}
$$

where $X_{i j}^{I}$ transforms in the representation $(\mathbf{N}, \overline{\mathbf{N}})$ of $U(N)_{i} \times U(N)_{j}$. These interact via a superpotential descended from the $\mathcal{N}=4$ theory

$$
\begin{aligned}
W= & \operatorname{tr}\left(Z_{12}\left(X_{23} Y_{31}-Y_{24} X_{41}\right)+Z_{21}\left(X_{14} Y_{42}-Y_{13} X_{32}\right)+\right. \\
& \left.Z_{34}\left(X_{41} Y_{13}-Y_{42} X_{23}\right)+Z_{43}\left(X_{32} Y_{24}-Y_{31} X_{14}\right)\right) .
\end{aligned}
$$

As discussed above, the gauge symmetry is in fact broken to $S U(N)^{4}$. The model has a non-anomalous global symmetry group

$$
G=\left(U(1)^{3} \times U(1)^{3} \rtimes S_{4}\right) \times \mathbb{Z}_{2} .
$$

The first $U(1)^{3}$ factor is the (effective) baryon number symmetry (recall that the diagonal acts trivially). As discussed above, this arises from $R R$ symmetries in the $A d S$ model. The second $U(1)^{3}$ factor acts by phases on the chiral multiplets, and we can choose a basis of generators acting on chiral multiplets arising from one of the complex coordinates with charge one.

One linear combination of the latter three $U(1)$ 's (the diagonal) is anomalous, and combines with the anomalous $U(1)_{R}$ symmetry of the model (under which all lowest components of superfields are fixed, gluinos have charge 1 and quarks charge -1$)$ to a non-anomalous $U(1)_{R}^{\prime}$ subgroup of $G$, under which the squarks all have charge $2 / 3$. The $S_{4}$ permutes the four factors of the gauge group. It also acts on the twelve chiral multiplets, permuting these as dictated by the index structure. For example, the permutation (12) exchanges $X_{14}$ with $Y_{24}$ and $X_{23}$ with $Y_{13}$ (as well as the fields in the conjugate representations), and $Z_{12}$ with $Z_{21}$. The superpotential is not invariant under $S_{4}$, so odd permutations must be combined with a $U(1)_{R}$ rotation by $\frac{\pi}{2} \cdot{ }^{25}$ The final $\mathbb{Z}_{2}$ factor in $G$ acts on the vector multiplets by charge

\footnotetext{
${ }^{25} \mathrm{~A}$ potential anomaly in this symmetry, along with the reason for its cancellation, was pointed out in [30]. We are being somewhat imprecise here; we restrict attention to the action on bosons. Thus we ignore the fact that $e^{i \pi R}=(-1)^{F}$ is not the identity.
} 
conjugation and exchanges $X_{i j}^{I}$ with $X_{j i}^{I}$. This too changes the sign of $W$ so must be combined with a $U(1)_{R}$ rotation by $\frac{\pi}{2}$. Thus, a $U(1)^{5} \rtimes A_{4}$ subgroup are not $R$-symmetries. The quantum symmetry discussed above is a $\mathbb{Z}_{2} \times \mathbb{Z}_{2}$ subgroup of $G$. As we shall see, this is precisely the subgroup of $S_{4}$ which permutes the chiral fields in (5.12) along rows only. More abstractly, it is the kernel of the natural map $S_{4} \rightarrow S_{3}$ giving the action on the spacetime indices and its nontrivial elements are products of disjoint two-cycles.

The moduli space of classical vacua is given by the solutions to the $F$ term equations following from (5.13) and the $D$-term equations, modulo $S U(N)^{4}$ gauge transformations. It is parameterized by the holomorphic gauge-invariants in the chiral superfields modulo the Jacobian ideal of (5.13) . These include meson-like operators constructed by finding products which transform in the adjoint representation of some $S U(N)$ factor and then taking traces of products of these. Since we are taking the trace, products which are related by cyclic permutations of the factors will lead to identical invariants. Modulo the equations of motion following from (5.13), these invariants are generated by

$$
\begin{array}{lll}
x_{1}=X_{14} X_{41} & y_{1}=Y_{13} Y_{31} & z_{1}=Z_{12} Z_{21} \\
x_{2}=X_{23} X_{32} & y_{2}=Y_{24} Y_{42} & z_{2}=Z_{34} Z_{43}
\end{array}
$$

and

$$
a=X_{14} Y_{42} Z_{21}
$$

where $a$ is the unique cubic adjoint in the sense that when traces are taken all cubics are equal modulo the equations of motion. In addition, there are baryon-like operators formed, as discussed above, by antisymmetrizing on both gauge indices a product of $N$ bifundamental fields. This yields twelve baryonic invariants $B_{i j} \sim X_{i j}^{N}$, subject to relations of the form (suppressing numerical factors)

$$
\begin{array}{lll}
B_{14} B_{41}=x_{1}^{N} & B_{13} B_{31}=y_{1}^{N} & B_{12} B_{21}=z_{1}^{N} \\
B_{23} B_{32}=x_{2}^{N} & B_{24} B_{42}=y_{2}^{N} & B_{34} B_{43}=z_{2}^{N} .
\end{array}
$$

Together with (5.15) these give coordinates on the full moduli space.

To forge the connection to the construction of [102] we note that the baryonic $U(1)^{3}$ symmetry acts on the classical moduli space $\mathcal{M}(N)$ preserving the symplectic structure. We can thus construct, if we wish, the symplectic reduction by this group action (or equivalently a holomorphic quotient by the complexified group action). The symplectic reduction is determined by a choice of the values of the moment map $\mu: \mathcal{M}(N) \rightarrow \mathbb{R}^{3}$. This reduction is equivalent to computing the moduli space of a theory with the same matter content but a larger gauge group $U(N)^{4} / U(1)$. The components in $\mathbb{R}^{3}$ of the 
moment map correspond to the values $\zeta_{i}$ of the three independent FayetIliopoulos $D$-terms which enter this latter construction. Thus, $\mathcal{M}(N)$ fibers over $\mathbb{R}^{3}$ with the fiber over $\zeta$ being a $U(1)^{3}$ bundle over the reduced space $\mathcal{M}_{0}(N ; \zeta)$. In view of our discussion above, the transition from $\mathcal{M}_{0}$ to the full moduli space can be reinterpreted as reinstating the baryonic invariants. We thus restrict attention to (5.15), and notice that these commute (when traces are taken) using (5.13), and satisfy the relations

$$
x_{i} y_{j} z_{k}=a^{2} .
$$

Note that if we treat the variables as scalars then the solution of $x y z=a^{2}$ is $\mathcal{M}_{0}(1 ; 0)=\mathbb{C}^{3} / \mathbb{Z}_{2} \times \mathbb{Z}_{2}$, precisely our transverse space.

The moduli space $\mathcal{M}(N)$ has several branches. ${ }^{26}$ At generic (nonzero) $x, y, z$, the equations of motion imply that $x_{i}^{I}=x_{j}^{I}$ and we can drop the subscripts. Thus on this branch of the moduli space the meson-like invariant traces parameterize the space of $S_{N}$ invariants of $N$ numbers (roots of the characteristic polynomial) satisfying (5.17), i.e., parameterize the space $\mathcal{M}_{0}(1 ; 0)^{N} / S_{N}$ which we can identify with $\mathcal{M}_{0}(N ; 0)$. The other level sets of $\mu$ (which together fill out the full moduli space) will be $U(1)^{3}$ bundles over $\mathcal{M}_{0}(N ; \zeta)$ which - as is familiar from toric geometry - will be related to partial resolutions of $\mathcal{M}_{0}(N ; 0)$. (The $\zeta$ 's serve to label the blowup moduli.) In fact, given the structure of the meson-like invariant traces we can identify $\mathcal{M}_{0}(N ; \zeta)$ with $\mathcal{M}_{0}(1 ; \zeta)^{N} / S_{N}$. This is precisely the configuration space for motion of $N$ branes on the (partially resolved) space $\mathcal{M}_{0}(1 ; \zeta)$. At generic points on this branch, the gauge symmetry is broken to $U(1)^{N-1}$, and the only massless matter consists of $3(N-1)$ neutral chiral multiplets, with no superpotential interaction. Thus the model has an accidental $\mathcal{N}=4$ supersymmetry and is precisely the low-energy description of the motion of $N$ branes at generic (smooth) points.

The other branches of the moduli space are found by setting, say, all $y_{i}=z_{i}=0$. Then the meson-like invariants restricted to this branch are generated by the two $x_{i}$ coordinates and parameterize a space $\left(\mathbb{C}^{N} / S_{N}\right)^{2}$, meeting the previous branch along the diagonal in the square. Along this branch $y$ and $z$ are massive. The gauge symmetry is broken to $U(1)^{2(N-1)}$. The interpretation of this branch following $[113,102,14,114]$ is that the $N$ branes have split into $2 N$ "fractional" branes, each with one-half the tension of a D3-brane. These are in fact D5-branes wrapped about a vanishing cycle (there is precisely one such cycle at generic points along the $x$ axis). The two D5-branes wrap the two-cycle with opposite orientations; the total six-form charge thus vanishes. The D5-branes do, however, acquire a fourform charge through the Chern-Simons coupling, equal to $1 / 2$ the charge of

\footnotetext{
${ }^{26}$ This phenomenon was anticipated by Sardo Infirri [112], who pointed out that for non-isolated quotient singularities, the construction of [112] (the one applied in [102]) will yield a space satisfying the $F$-flatness conditions which has several components.
} 
a fundamental D3-brane (and a tension in four dimensions determined by this charge and the BPS condition they satisfy). Notice that these wrapped branes are not able to move off in the $y$ and $z$ directions, in accordance with the fact that $y$ and $z$ are massive along this branch. Also, the restriction on the $\zeta_{i}$ above is in line with our interpretation of these as blowup moduli for the singularity. Along the "fractional" branch the cycle wrapped by the D5-branes is constrained to remain at zero size. Of course, two more such branches exist along the $y$ and the $z$ axes. Also, there are mixed branches in which some of the $N$ branes have split along each of the axes and some have remained unsplit and hence free to move off in any direction. At the origin of moduli space the nature of the objects is ambiguous, since they are free to move off on either branch. They are perhaps best described as bound states of D3-branes and wrapped D5-branes [114] .

From these pieces we can now assemble our total moduli space of vacua, as a fibration over the space of $\zeta$ values. At generic values we find a $U(1)^{3}$ bundle over the space $\mathcal{M}_{0}(N ; \zeta)$ which is the configuration space of $N$ points moving on the resolved singularity with blowup parameters $\zeta$. In codimension one, whenever the sum of two $\zeta_{i}$ vanishes, the fiber has an additional branch, meeting the previous branch along a complex curve in the base of the $U(1)^{3}$ bundle. Along this new branch we find a $U(1)^{2}$ fibration ${ }^{27}$ over $\left(\mathbb{C}^{N} / S_{N}\right)^{2}$. The moduli space of vacua thus contains both the moduli of the singularity and those describing motion of the branes along it.

The utility of this indirect construction is the following. We have seen that for $N=1$ (5.15) parameterize the transverse space. The toric methods of [102] allow us to pursue this identification further; we can in fact identify directly a map from the FI parameters $\zeta_{i}$ appearing in the construction and the blowup moduli of the singularity (see appendix B for details of this as applied to this example, following [103] .) In this paper, our interest in this quotient singularity is in finding the loci in its classical moduli space at which nonabelian gauge groups are unbroken, and associating these to special partial resolutions of the singularity which contain nontrivial singular points. We then identify these gauge theories as the worldvolume theory for branes at the corresponding singularities. The point is, that in the toric construction one finds singularities for those values of $\zeta_{i}$ for which the moduli space $\mathcal{M}_{0}(1 ; \zeta)$ contains a point at which some Abelian gauge group remains unbroken. For these values, $\mathcal{M}_{0}(N ; \zeta)$ will contain points with unbroken $U(N)$ symmetries. In particular, this corresponds to a point in $\mathcal{M}(N)$ with unbroken $S U(N)$ gauge symmetry. This argument allows us to forge a link between the toric analysis of $\mathcal{M}_{0}(1, \zeta)$ using the trick of [102] and the classical moduli space of the field theory. The worldvolume theory

\footnotetext{
${ }^{27}$ Geometrically one of the $S^{1}$ directions in the fiber is absent along this branch because the cycle about which it measured the four-form period is absent. More directly, when the modulus $\zeta$ of the complex scalar vanishes its phase becomes irrelevant.
} 
for branes at the singularities arising from the partial resolution determined by some particular value of $\zeta_{i}$ will be the low-energy theory determined by the corresponding point in the moduli space of the theory we are treating here.

The model we have described is indeed a conformal field theory. The one-loop $\beta$-functions for the $S U(N)$ couplings all vanish, and the cubic superpotential is a marginal deformation at weak coupling. We can confirm this result beyond weak coupling using the methods of [115]. The existence of marginal operators can be tested non-perturbatively by studying the "scaling coefficients" determining the $\beta$-functions for various couplings in terms of the anomalous dimensions [116, 117] . Making some assumptions on the genericity of the latter as functions of the couplings we can deduce the dimension of the critical subspace in coupling space from linear dependencies among the coefficients.

Eq. (5.13) is the unique superpotential preserving the entire global symmetry group. If we preserve the symmetry, the model has only two independent couplings - the gauge coupling and the superpotential coefficient. These are written in terms of the anomalous dimension of the chiral fields (when the symmetry is unbroken all the dimensions are of course equal)

$$
\begin{aligned}
& A_{g}=-6 N \gamma \\
& A_{h}=3 \gamma / 2 .
\end{aligned}
$$

Clearly, $\gamma=0$ leads to a conformal theory. This imposes one condition on the two couplings leaving a line of fixed points enjoying the full global symmetry. We can parameterize these theories by $\tau=\frac{\theta}{2 \pi}+\frac{4 \pi i}{g^{2}}$; the superpotential coupling will now be determined by the requirement of conformal invariance and are not an additional marginal coupling.

We can look for additional marginal operators by relaxing the symmetry requirements. If we require only that $U(1)_{R}$ and charge conjugation (together with the exchange of $X_{i j}^{I}$ with $X_{j i}^{I}$ ) be symmetries, we can parameterize the most general gauge-invariant superpotential. This has eight possible terms (all of which appear in our $W$ ). These pair up into four pairs related by charge conjugation as above. Together with the four gauge couplings we thus have eight coefficients written as functions of the six independent anomalous dimensions

$$
\begin{aligned}
& A_{g_{1}}=-2 N\left(\gamma_{12}+\gamma_{13}+\gamma_{14}\right) \\
& A_{g_{2}}=-2 N\left(\gamma_{12}+\gamma_{23}+\gamma_{24}\right) \\
& A_{g_{3}}=-2 N\left(\gamma_{13}+\gamma_{23}+\gamma_{34}\right) \\
& A_{g_{4}}=-2 N\left(\gamma_{14}+\gamma_{24}+\gamma_{34}\right) \\
& A_{h_{1}}=\frac{1}{2}\left(\gamma_{12}+\gamma_{23}+\gamma_{13}\right)
\end{aligned}
$$




$$
\begin{aligned}
& A_{h_{2}}=\frac{1}{2}\left(\gamma_{12}+\gamma_{24}+\gamma_{14}\right) \\
& A_{h_{3}}=\frac{1}{2}\left(\gamma_{13}+\gamma_{14}+\gamma_{34}\right) \\
& A_{h_{4}}=\frac{1}{2}\left(\gamma_{23}+\gamma_{24}+\gamma_{34}\right),
\end{aligned}
$$

where $\gamma_{i j}$ is the anomalous dimension of $X_{i j}^{I}$ and $X_{j i}^{I}$. The notation is such that $S_{4}$ acts on the indices of both $g$ and $h$. These are easily seen to satisfy four linear relations. Thus, the vanishing of all of (5.19) imposes only four conditions on the six anomalous dimensions. In terms of the eight original couplings we thus predict a four (complex) dimensional space of fixed points, of which a one-dimensional subspace preserves the full $S_{4}$ symmetry, while at a symmetric point we have three truly marginal deformations permuted by $S_{3}$. The $S_{4}$ structure then guarantees that these are charged under the quantum $\mathbb{Z}_{2} \times \mathbb{Z}_{2}$ symmetry with charges,+--+ , and --. We can parameterize the entire fixed space by the four gauge couplings $\tau_{i}$, which are permuted by the action of $S_{4}$. The symmetric subspace on which all of the couplings are equal is parameterized by $\tau_{++}=\sum \tau_{i}$, and we can form combinations with the weights listed above

$$
\begin{aligned}
& \tau_{+-}=\tau_{1}+\tau_{2}-\tau_{3}-\tau_{4} \\
& \tau_{-+}=\tau_{1}-\tau_{2}+\tau_{3}-\tau_{4} \\
& \tau_{--}=\tau_{1}-\tau_{2}-\tau_{3}+\tau_{4},
\end{aligned}
$$

where the subscripts indicate the charges with which these transform under our chosen generators for the quantum symmetry, represented in $S_{4}$ by

$$
S_{1}=(12)(34) \quad \text { and } \quad S_{2}=(13)(24) .
$$

For comparison with the $A d S$ model we mention one more property of the model that is easily probed. Chiral primary operators are given by gaugeinvariant polynomials in the chiral fields modulo descendants; the latter are expressed as the Jacobian ideal of $W$. For these operators the scaling dimension is determined by the $R$-charge $\Delta=\frac{3}{2} R$, hence protected from quantum corrections (provided we use the correct $R$-symmetry). Below we list some of these operators. For comparison to the $A d S$ picture, we also give their transformation properties under the global symmetry group. We label the operators by their charges under the continuous global symmetry of (5.14) and under the quantum symmetry group. We restrict attention to scalars with conformal dimension $\Delta \leq 4$, except for the baryonic states which are not part of the towers of operators constructed over the low-lying ones.

1. The kinetic energy operators for the four gauge fields $\operatorname{tr} F_{i}^{2}$ are marginal $(\Delta=4)$ operators as discussed above. They are permuted by $S_{4}$ and 
are all invariant under $U(1)^{3}$. Since they are permuted by $S_{4}$ we can form one invariant combination, and three others transforming in onedimensional representations of the discrete symmetry. Explicitly, these are

$$
\begin{aligned}
f_{++} & =\frac{1}{4} \sum_{i=1}^{4} \operatorname{tr} F_{i}^{2} \\
f_{--} & =\frac{1}{4} \operatorname{tr}\left(F_{1}^{2}-F_{2}^{2}-F_{3}^{2}+F_{4}^{2}\right) \\
f_{-+} & =\frac{1}{4} \operatorname{tr}\left(F_{1}^{2}-F_{2}^{2}+F_{3}^{2}-F_{4}^{2}\right) \\
f_{+-} & =\frac{1}{4} \operatorname{tr}\left(F_{1}^{2}+F_{2}^{2}-F_{3}^{2}-F_{4}^{2}\right),
\end{aligned}
$$

coupling to the combinations of $\tau_{i}$ as in (5.20). These couplings are distinguished, as found above, as being exactly marginal.

2. The meson operators $\operatorname{tr}\left(X_{i j}^{I} X_{j i}^{I}\right)$, with $\Delta=2$. There are six operators of this type. The operators of charge $(2,0,0)$ under $U(1)^{3}$ transform in a two-dimensional representation of $S_{4}$. Forming linear combinations we find

$$
\begin{aligned}
& x_{++}=\operatorname{tr}\left(X_{14} X_{41}+X_{23} X_{32}\right) \\
& x_{--}=\operatorname{tr}\left(X_{14} X_{41}-X_{23} X_{32}\right) .
\end{aligned}
$$

Similarly, we find the operators $y_{++}$and $y_{-+}$with charge $(0,2,0)$ and $z_{++}$and $z_{+-}$with charge $(0,0,2)$.

3. The operators $\operatorname{tr}\left(W_{\alpha} W^{\alpha}\right)_{i}$ with $\Delta=3$. These are permuted by $S_{4}$, so we form linear combinations as in (5.22) above. These all have $R$-charge 2 but their $U(1)^{3}$ charges must follow from anomaly cancellation, and one finds easily

$$
\begin{array}{llll}
w_{++} & (1,1,1) & w_{-+} & (0,3,0) \\
w_{--} & (3,0,0) & w_{+-} & (0,0,3) .
\end{array}
$$

4. The operator $d=\operatorname{tr}\left(X_{i j} Y_{j k} Z_{k i}\right)$, with dimension $\Delta=3$. Note that modulo descendants there is precisely one such operator. It is invariant under the quantum symmetry and carries charge $(1,1,1)$.

5. The operators $\operatorname{tr}\left(X_{i j}^{I} X_{j k}^{J} X_{k l}^{I} X_{l i}^{J}\right)$, with $\Delta=4$. Modulo descendants, there is one operator of type ++ for each pair of superscripts. To form nontrivial representations of the discrete group we find that we must have $I=J$ and thus we have

$$
\begin{array}{ll}
q_{++}^{I J} & (4,0,0),(0,4,0),(0,0,4),(2,2,0),(2,0,2),(0,2,2) \\
q_{+-} & (0,0,4) \\
q_{-+} & (0,4,0) \\
q_{--} & (4,0,0) .
\end{array}
$$


6. Finally, because the gauge group is $S U(N)^{4}$, we can construct baryon operators of the form

$$
B_{i j}=\epsilon^{a_{1} \cdots a_{N}} \epsilon_{b_{1} \cdots b_{N}}\left(X_{i j}^{I}\right)_{a_{1}}^{b_{1}} \cdots\left(X_{i j}^{I}\right)_{a_{N}}^{b_{N}},
$$

with $I$ determined by $i j$. The $S_{4}$ transformation properties, like those of the $X^{I}$, are determined by the index structure. Thus, for each $I$ we can form two baryons and two antibaryons. Since baryon number is broken in this theory the latter term requires clarification. It is appropriate in the sense that a baryon-antibaryon state can decay to mesonic states. Constructing linear combinations with definite charges under the quantum symmetry, we find in each sector a baryon and an antibaryon. In the twisted sectors these have charges $(N, 0,0)_{--}$, $(0, N, 0)_{-+}$, and $(0,0, N)_{+-}$. In the untwisted sector we find all three charge configurations.

\subsection{The $A d S$ compactification}

The dual AdS theory is found following [12]. We utilize the construction of [102] once more, constructing the theory as a quotient of the theory with $4 N$ branes. But in this case, we first use the original $A d S / C F T$ duality to map this latter to a IIB compactification on $A d S_{5} \times S^{5}$. We then implement the quotient in this dual theory. It is clear that since we are taking the quotient by a subgroup of $S O(6)$, this acts purely on the $S^{5}$, leaving the $A d S$ space untouched. Thus the resulting theory is indeed of the form $A d S_{5} \times H^{5}$, predicting a conformal field theory on the branes. The horizon is in this case simply

$$
H^{5}=S^{5} /\left(\mathbb{Z}_{2} \times \mathbb{Z}_{2}\right)
$$

which is singular along three circles. As discussed in 5.1, we expect to find three twisted sectors corresponding to these fixed loci.

In section 4 , the group of supersymmetric isometries of the quotient was computed to be $U(1)^{3} \rtimes S_{3}$. The $U(1)$ factors correspond to rotation by a phase of each of the three complex coordinates, while the $S_{3}$ permutes these. Each of the generators of the continuous group acts as a rotation on one of the fixed circles; the discrete group permutes the three. These are gauge symmetries in the $A d S$ compactification and will couple to the global symmetry currents in the boundary $C F T$. We can ask which of these are $R$-symmetries. To answer this, note that our one supersymmetry generator, out of the four in the covering theory, is the invariant spinor in the decomposition of the $S O(6)$ spinor representation under $S U(3) 4=\mathbf{3} \oplus \mathbf{1}$. The $R$-symmetries are then those transformations which act (by phases) on this representation. This immediately shows that the diagonal $U(1)$ and all odd permutations in $S_{3}$ are $R$-symmetries, and indeed predicts the correct action on the supercharges. 
Thus the isometry group of $H^{5}$ contains the $R$-symmetry of the $C F T$ as shown in general in section 3, as well as a part of its global symmetry, but not all of the latter. First of all, the baryonic symmetry is not realized as an isometry. As in our general discussion of quotient singularities, this corresponds to a gauge symmetry in the $A d S$ theory carried by periods of the RR four-form about three-cycles in $H^{5}$. These are constructed along the lines of the discussion for non-isolated singularities. The structure of $H^{5}$ transverse to each of the three singular circles is $\mathbb{C}^{2} / \mathbb{Z}_{2}$, containing in its resolution a two-cycle $\Sigma_{i}$. These lead to nontrivial three-cycles in $H^{5}$ of the form $C_{i}=\Sigma_{i} \times S_{i}^{1}$. The periods of the RR four-form about these yield the gauge fields for a $U(1)^{3}$ symmetry corresponding precisely to the baryon number symmetry in the worldvolume, as in our general discussion of quotients above.

Second, there are still discrete group actions we have not accounted for by isometries. The first of these, as expected, is the quantum $\mathbb{Z}_{2} \times \mathbb{Z}_{2}$ action under which the untwisted sector states are uncharged, while the twisted sector states have charges $(--),(-+)$, and $(+-)$. It is gratifying to find that the extension of $S_{3}$ by this action is precisely $S_{4}$, as discussed above. Having now found this, we now note that if we find three objects permuted by $S_{3}$ then their charges under the quantum symmetry must be the ones listed above, since this is the only situation that arises from $S_{4}$ representations. The additional $\mathbb{Z}_{2}$ associated to charge conjugation is also not evident as an isometry. Charge conjugation is associated to orientation reversal for the open strings in the theory, and this symmetry is mapped under the correspondence to the action of the center of the $S L(2, \mathbb{Z})$ duality group of IIB string theory. It thus commutes with all other actions. As shown in [30], this action must be accompanied by a nontrivial $R$-transformation. Thus the $A d S$ picture reproduces exactly the global symmetry group found in the $C F T$ description.

Having matched the global symmetries we move on to compare the moduli of the two theories (in the sense of exactly marginal couplings). The type IIB compactification always has a universal marginal coupling, the string coupling $\tau_{S}=\frac{\chi}{2 \pi}+\frac{2 \pi i}{g}$, where $\chi$ is the RR scalar. In a compactification on $A d S_{5} \times S^{5}$ this is the only modulus, and the gauge coupling is simply given by the scaling argument as $\tau_{++}=\tau_{S}$, determined by the asymptotic value of the dilaton far from the branes. Extra moduli in this example must thus come from the twisted sectors, and indeed there are three of these. Each has the structure (transverse to the fixed circle) $\mathbb{C}^{2} / \mathbb{Z}_{2}$ which will, according to our general discussion, produce precisely one marginal deformation, given by the periods of the two-form potentials about the one two-cycle in the resolution of this singularity. We label these moduli $b_{ \pm \pm}$according to their sectors; the global symmetry and the scaling argument then determine 


$$
\begin{aligned}
\tau_{++} & =\tau_{S} \\
e^{2 \pi i \tau_{ \pm \pm}} & =f\left(e^{2 \pi i b_{ \pm \pm}}\right)
\end{aligned}
$$

where in the second line the combination ++ does not appear. We note that the couplings $b$ here should be interpreted as the deviation of the periods from their values at the $\mathbb{Z}_{2} \times \mathbb{Z}_{2}$ invariant orbifold point, i.e. $1 / 2$. One interesting feature of this mapping is that the periods naturally parameterize a twotorus. This periodicity in principle predicts that the $\mathcal{N}=1$ theory that we are studying has an $S$-duality! Extracting a precise form of this prediction would require knowledge of the function $f$ above, however. In [14] this predicted type of duality was shown to agree with the known $S$-duality of $\mathcal{N}=2$ orbifold models.

As a final check of the conjecture we compare the spectrum of conformal dimensions in the $C F T$ to the mass spectrum in the $A d S$ model. In this paper, we restrict attention to relevant and marginal scalars. A scalar field of conformal dimension $\Delta$ on the boundary couples [7] to a scalar field of mass $m^{2}=\Delta(\Delta-4)$. In the untwisted sector the matching spectra are no surprise, since fundamentally both follow by projection from the spectra in the covering theory, and the latter are known to agree. The twisted-sector fields can be studied using the methods of $[23,9]$. The twisted sector fields propagate in six dimensions along $A d S_{5} \times S^{1}$. Precisely this situation was studied in [23]. (That this was done there in the context of $\mathcal{N}=2$ models is irrelevant; in the twisted sector as formulated here no further projection is required and low-lying twisted states are sensitive only to the local properties of the space.) The spectrum of chiral states is found by Kaluza-Klein reduction on $S^{1}$ of the fields in the well-known six-dimensional theory associated to a $\mathbb{C}^{2} / \mathbb{Z}_{2}$ singularity, modified by the presence of curvature and five-form flux. The resulting five-dimensional spectrum corresponds to $\mathcal{N}=2$ supersymmetry in the four-dimensional $C F T$. The states are thus labeled by their charges under the $S U(2) \times U(1) R$-symmetry, where the $S U(2)$ is the six-dimensional $R$-symmetry and the $U(1)$ represents rotations of the fixed circle. In our case, considering, say, the twisted sector associated to $y=z=0$ (which we previously denoted the -- sector) we will find that since $Y, Z$ are massive the light states will all be invariant under two of the $U(1)^{3}$ generators. Thus there is only one $U(1)$ action in this sector, which will be a linear combination of $J_{S^{1}}$ and $J_{3}$. We identify

$$
J_{R}=\frac{4}{3} J_{3}+\frac{1}{3} J_{S^{1}}
$$

Thus the $U(1)^{3}$ charges of all the states in this sector will be of the form $\left(\frac{3}{2} R, 0,0\right)$. Note that all the twisted states predicted above indeed have charges of this form. 
Gukov finds the following light scalars in the twisted sector. There are fields with $m^{2}=-4$ in the $\mathbf{3}_{0}$. Using (5.29), we see that only one of these has the right $R$-charge, and thus we find the field coupling to $x_{--}$. There is also a triplet of $m^{2}=-3$ transforming as $\mathbf{3}_{2}$. Here again only one field has the correct $R$-charge and couples to $w_{--}$of (5.24) . There is yet another set at $m^{2}=0$, transforming as $\mathbf{3}_{4}$. This too leads to a unique chiral primary coupling to $q_{--}$of (5.25). All of these come from the multiplet of blowup modes. From the two-form period multiplet, we find one marginal field, a singlet under the global symmetry. We conjecture that this couples to the exactly marginal operator $f_{--}$, in accordance with our discussion of the moduli above.

Finally, we consider the spectrum of wrapped D3-branes in this model. As discussed in 5.1, these will correspond to the twisted baryonic operators. The three-cycles about which we propose to wrap D3-branes will be the $C_{i}$ mentioned above. The antibaryons will be obtained by wrapping $-\Sigma_{i}^{*} \times S_{i}^{1}$. In each sector, according to the discussion above, the charges are restricted to a one-dimensional subgroup of $U(1)^{3}$ and the total charge can be determined from the mass as predicted by (5.3) above. The charges under the baryon number symmetry follow from the intersection between the two- and threecycles in $H^{5}$. This yields precise agreement with the computation in the CFT.

\section{New examples in four dimensions}

Our choice of the $\mathbb{Z}_{2} \times \mathbb{Z}_{2}$ quotient construction in the previous section was motivated by the fact that several interesting singularities can be found by considering partial resolutions of this particular quotient. This was pointed out recently in $[103,104]$, where the methods of $[102]$ were used to show that the moduli space of deformations included conifold transitions. Note that [102] studies the case of a single brane $(N=1)$. In this case, according to our discussion in section 5 , the gauge symmetry will be completely broken. Nevertheless it is possible to study the moduli space as a fibration of torus bundles over moduli spaces of a family of auxiliary Abelian gauge theories (obtained by gauging the baryon number symmetries), parameterized by the space of FI terms. These auxiliary moduli spaces have descriptions as toric varieties [102]. This is a nontrivial result as it requires that the $F$ term equations be expressed in terms of toric data. We have seen that the fiber thus described is in fact a neighborhood of the singularity in the internal manifold. In a sense, the baryonic degrees of freedom ignored in this construction represent the moduli of the singularity, while the mesonlike $U(N)$ invariants parameterize the positions of the branes. Thus this description leads directly to a description of the horizon geometry. Also, the Kähler class of the moduli space is explicitly parameterized by the FI terms 
introduced. This enables a direct determination of loci in the moduli space at which the low-energy theory is nontrivial, as well as the corresponding singularities in the partial resolutions of the orbifold.

The way this works in practice is the following. By choosing values for the parameters $\zeta_{i}$ of the previous section we select a point in the moduli space of the field theory. We can then study the low-energy theory about this vacuum. At generic points, the result as mentioned above is the $\mathcal{N}=4$ theory of branes at smooth points. There will be special values of $\zeta$ which lead to different low-energy limits. In the $A d S$ picture we need to recall that low-energy physics on the worldvolume is related to small-distance physics in $A d S_{5}$ [5, 118]. Thus, the flow to the low-energy limit corresponds to shrinking the horizon manifold. We thus find that the special points in moduli space are precisely those for which a small neighborhood is nonspherical - those points corresponding to locating the branes at singularities of the partially-resolved space. The latter were described in section 4 (and appendix B) following [102], and in this section we will use this tool to investigate the conjectures for these singularities.

This makes possible a direct computation of the low-energy theory. The horizon manifolds were described in section 4 . What checks can we perform on the conjectures for these examples? The first test is a comparison of the global symmetries of the field theory to the isometry groups found geometrically. As we have seen above, in general there will be other sources for global symmetries. The baryonic symmetries will correspond to the periods of the four-form. In orbifold models there will also be discrete quantum symmetries. Finally, our derivation will lead to models in which the two-form periods are one-half their maximum value, thus preserving the $\mathbb{Z}_{2}$ center of $S L(2, \mathbb{Z})$ which will appear in the field theory as a charge-conjugation symmetry.

As a second check we can try to compare the spectrum of chiral primary operators in the $C F T$ to the spectrum of states in the $A d S$ compactification. In general, the former is an easy exercise but the latter is difficult for the horizon manifolds in question. What we will find relatively easy to describe is the spectrum of wrapped D3-brane states corresponding to baryons. Requiring only an understanding of the topology of $H^{5}$ and some assumptions on the form of the metric (and hence on the parameter spaces of minimalvolume representatives of certain homology cycles which we have already determined in section 4.3), this will in fact be a tractable problem and we will make this comparison for all the models we study.

Finally, we can compare marginal operators in the field theory to moduli of the $A d S$ model. Here we will find an extension of the pattern we observed in the quotient models. For small resolutions (blowups in which each singular point is replaced by a curve) there will be one marginal operator, 
corresponding to the two-form periods, and the blowup parameter will appear as an FI parameter (more precisely as the baryonic mode corresponding to this). For a large blowup (in which a point is replaced by a surface) we will find one marginal operator again, given by two-form periods, and associated to two FI terms subject to a reality condition like (5.4).

We turn now to the four singularities discussed in section 4.3 ; for each example we compute the worldvolume theory at low energies and compare to the $A d S$ predictions.

\subsection{The $\mathbb{Z}_{2}$ quotient singularity}

The toric analysis shows that setting, say, $\zeta_{1}+\zeta_{2}=0$ corresponds to leaving one of the $\mathbb{Z}_{2}$ singularities unresolved. In the scaling limit we thus expect to find the $\mathcal{N}=2$ theory associated to the quotient $\mathbb{C}^{2} / \mathbb{Z}_{2}$.

The most symmetric vacuum in this level set of the moment map is represented by

$$
\begin{aligned}
& Z_{12}=\zeta_{1}^{1 / 2} \\
& Z_{43}=\zeta_{4}^{1 / 2}
\end{aligned}
$$

all others vanishing. The gauge symmetry is broken to $S U(N) \times S U(N)$. The fields in (6.1) are eaten by the Higgs mechanism, while (5.13) shows that $Y_{24}$ and $Y_{31}$, say, get $F$-term masses. The equations of motion follow from (5.13)

$$
\begin{aligned}
& \zeta_{1}^{1 / 2} X_{23}=\zeta_{4}^{1 / 2} X_{14} \\
& \zeta_{1}^{1 / 2} X_{41}=\zeta_{4}^{1 / 2} X_{32}
\end{aligned}
$$

Inserting these and integrating out the massive fields, we find that the light fields and their charges can be taken $\operatorname{as}^{28}$

$$
\begin{array}{lllllll}
x_{1}=X_{14} & (\mathbf{N}, \overline{\mathbf{N}}) & x_{2}=Y_{13} & (\mathbf{N}, \overline{\mathbf{N}}) & \phi_{1}=Z_{21} & \left(\mathbf{N}^{2}-\mathbf{1}, \mathbf{1}\right) \\
\tilde{x}_{1}=Y_{42} & (\overline{\mathbf{N}}, \mathbf{N}) & \tilde{x}_{2}=X_{32} & (\overline{\mathbf{N}}, \mathbf{N}) & \phi_{2}=Z_{34} & \left(\mathbf{1}, \mathbf{N}^{2}-\mathbf{1}\right)
\end{array}
$$

and plugging (6.2) in we find the superpotential

$$
W=\zeta^{-1 / 2} \operatorname{tr}\left(\left(\zeta_{1}^{1 / 2} \phi_{1}-\zeta_{4}^{1 / 2} \phi_{2}\right)\left(x_{1} \tilde{x}_{1}-x_{2} \tilde{x}_{2}\right)\right)
$$

\footnotetext{
${ }^{28}$ Note that $\mathbf{N}$ is the fundamental representation and $\overline{\mathbf{N}}$ its complex conjugate; $\mathbf{N}^{2}-\mathbf{1}$ is the adjoint representation.
} 
where $\zeta=\zeta_{1}+\zeta_{4}$. Note that the trace of $Z$ is given a mass by the supersymmetric completion of the couplings which break the Abelian gauge symmetry.

The theory is observed to be in fact precisely an $\mathcal{N}=2$ theory with the gauge group above and two hypermultiplets in the $(\mathbf{N}, \overline{\mathbf{N}})$ representation as expected. These cases have been studied in detail in $[12,14,23]$.

As in the previous section, we find a Higgs branch of the moduli parameterizing motion of the branes in the transverse directions, a Coulomb branch describing "fractional" branes constrained to move along the singular curve, and the expected mixed branches.

The dual IIB compactification on $A d S_{5} \times\left(S^{5} / \mathbb{Z}_{2}\right)$ has been studied in detail in, e.g., $[12,14,18,23]$. These works have provided evidence for the conjecture in this context, essentially along the lines of our discussion of the $\mathbb{Z}_{2} \times \mathbb{Z}_{2}$ quotient.

We can add here only that we expect baryons in this theory in accordance with our discussion in 5.1; the corresponding three-cycles arise as discussed there.

\subsection{The conifold}

As discussed in $[103,104]$ there is a different codimension-one locus in the space of FI parameters at which we obtain a conifold singularity. This occurs at, say, $\zeta_{1}=0$. To find the worldvolume theory, we take $\zeta_{2}, \zeta_{3} \gg 0$, maintaining $\zeta_{1}=0$. The most symmetric vacuum solution in this level set is represented by the expectation values

$$
\begin{aligned}
& Y_{24}=\zeta_{2}^{1 / 2} \\
& Z_{34}=\zeta_{3}^{1 / 2}
\end{aligned}
$$

all others zero. This breaks the gauge group down to $S U(N) \times S U(N)$. The Higgs mechanism eats the components of the multiplets with nonzero vevs. Inserting these expectation values into the superpotential leads to masses for some of the other fields, which are then integrated out by imposing their equations of motion. This leaves the light fields and their representation content as

$$
\begin{array}{lllll}
X_{1}=X_{14} & (\mathbf{N}, \overline{\mathbf{N}}) & Y_{1}=Y_{31} & (\overline{\mathbf{N}}, \mathbf{N}) \\
X_{2}=Z_{12} & (\mathbf{N}, \overline{\mathbf{N}}) & Y_{2}=Z_{21} & (\overline{\mathbf{N}}, \mathbf{N})
\end{array}
$$

where $Z_{21}$ represents the remaining massless degree of freedom after this field mixes with $Y_{13}$. Imposing the equations of motion for the massive fields 
we are left with the superpotential

$$
W=\frac{1}{2} \zeta^{-1 / 2} \operatorname{tr}\left(\epsilon^{i j} \epsilon^{k l} X_{i} Y_{k} X_{j} Y_{l}\right),
$$

with $\zeta=\zeta_{2}+\zeta_{3}$. Note that for $N=1$ we obtain precisely the charges and fields of (4.24). This will not be true in general, since the singularity is in general obtained at the origin of a moduli space determined by the gauge symmetry as well as the superpotential. It is a coincidence in this case that for $N=1$ (6.7) in fact vanishes.

This description agrees precisely with the one found independently in [30] by different methods. Our approach yields an explicit derivation of this field theory, including the superpotential proposed in [30].

The model has a global symmetry group $U(2) \times U(2)$, under which the fields transform as

$$
(\mathbf{N}, \overline{\mathbf{N}}, \mathbf{2}, \mathbf{1}) \oplus(\overline{\mathbf{N}}, \mathbf{N}, \mathbf{1}, \mathbf{2}) .
$$

The diagonal $U(1)$ in this is in fact anomalous, but this can be combined with the naïve $U(1)_{R}$ symmetry to yield a non-anomalous $U(1)_{R}^{\prime}$, under which all the lowest components of chiral superfields have charge $1 / 2$. The other $U(1)$ generator generates a non- $R$ baryonic symmetry, under which $X$ and $Y$ have opposite charges \pm 1 . There is an additional discrete $\mathbb{Z}_{2} \times \mathbb{Z}_{2}$ symmetry. We can take the generators to act as follows. One exchanges the two factors of the gauge group and acts on the chiral fields by exchanging $X$ and $Y$, and the other acts in the same way on the chiral multiplets but acts on the vector multiplets by charge conjugation. (Their product thus acts by exchanging the factors in the gauge group and charge conjugation, leaving the lowest components of the chiral multiplets untouched). Note that the first of these is in fact an $R$-symmetry, since the superpotential changes sign. The global symmetry group is thus

$$
G=U(2)^{2} \rtimes\left(\mathbb{Z}_{2} \times \mathbb{Z}_{2}\right) .
$$

Comparing this to (4.25) we see that as expected the baryonic symmetry as well as the discrete symmetry related to charge conjugation are not realized as isometries. The former corresponds to the four-form periods about the generator of $H_{3}$ and the latter to the center of $S L(2, \mathbb{Z})$ as in our general discussion.

The classical moduli space of vacua can be found here as above. The invariants are given by traces of the following four generators

$$
a_{i j}=X_{i} Y_{j}
$$

which inside traces all commute (using the equations of motion following from (6.7) ) and are subject to the relation

$$
a_{12} a_{21}=a_{11} a_{22} .
$$


To these we must add the baryonic invariants

$$
\begin{aligned}
& B_{s} \sim X_{1}^{s} X_{2}^{N-s} \\
& \tilde{B}_{s} \sim Y_{1}^{s} Y_{2}^{N-s}
\end{aligned}
$$

where both gauge indices are antisymmetrized, so that the flavor indices are symmetrized. These transform in the spin- $N / 2$ representation of the two $S U(2)$ global symmetry groups. They satisfy relations of the form

$$
B_{s} \tilde{B}_{t} \sim a_{11}^{s} a_{12}^{t-s} a_{22}^{N-t}
$$

for $s \leq t$.

As in the quotient singularity of section five, the meson-like invariant traces (6.10) parameterize the space of $S_{N}$ invariants in $N$ solutions of (6.11) - Comparing to the third line of Table 4, we see that this is precisely the $N^{\text {th }}$ symmetric product of the conifold singularity, as expected. Including the baryonic invariants (6.12) will, it is expected, lead to a classical moduli space fibered over the space of moduli of the singularity, with the fibers describing the locations of $N$ branes in the resolved space. The gauge symmetry is generically broken to $U(1)^{N-1}$. There are no "fractional" branches in this model. We do not expect such branches since the singularity is isolated.

We can identify the baryons in the spectrum of the CFT with particles in the $A d S$ compactification. As discussed above, these correspond to D3branes wrapped about three-cycles in $H^{5}$. The discussion of 4.3 shows that this is topologically just $S^{2} \times S^{3}$, so there is a natural identification of the three-cycle. In fact, there are two distinct ways to write $H^{5}$ as such a product, corresponding to the two $\mathbb{C P}^{1}$ components of the base of the $S^{1}$ fibration. These correspond to $B$ and $\tilde{B}$ of $(6.12)$. The space of three-cycles in either class is simply $S^{2}$, as discussed in section 4.3. The wavefunctions on this space will be sections of a line bundle of degree $N$ as determined by the five-form flux through $H^{5}$ [107] . Under the $U(1)_{B} \times S U(2) \times S U(2)$ isometry group, this will lead to spins $(0, N / 2)_{N}$ and $(N / 2,0)_{-N}$ in exact agreement with the predictions from the $C F T$.

The gauge theory with no superpotential is known to flow in the infrared to an interacting conformal fixed point (the nonabelian Coulomb phase). To analyze the situation in the presence of the superpotential we can use once more the methods of [115]. If we preserve the full global symmetry mentioned above, then the scaling coefficients for the two $S U(N)$ gauge couplings are identical; likewise, the anomalous dimensions for all the fields are the same. The superpotential is the unique one preserving the symmetry. The scaling coefficients are thus

$$
\begin{aligned}
& A_{g}=-2 N(1+2 \gamma) \\
& A_{h}=1+2 \gamma .
\end{aligned}
$$


Since they are proportional, we expect a (complex) line of fixed points. Note that in this case $\gamma=0$ is not a solution. The fixed points are all interacting and the line of fixed points does not extend out to weak coupling. Thus we have one modulus even under the $\mathbb{Z}_{2}$. We can once more relax the symmetry requirement, demanding only that $S U(2) \times S U(2)$ be unbroken. We then have two independent anomalous dimensions, and still only one superpotential coupling $h$. The scaling factors are now

$$
\begin{aligned}
A_{g_{1}} & =-2 N\left(1+\gamma_{X}+\gamma_{Y}\right) \\
A_{g_{2}} & =-2 N\left(1+\gamma_{X}+\gamma_{Y}\right) \\
A_{h} & =1+\gamma_{X}+\gamma_{Y} .
\end{aligned}
$$

Since in this case we have one condition on three couplings, there is an additional, $\mathbb{Z}_{2}$-odd, marginal operator. This is naturally identified with the difference of the two gauge couplings. More precisely, as discussed in [30] , for this theory we should form dimensionless combinations of $h$ and the dynamical scales (complexified by $\theta$ angles as usual), $\lambda_{i}=h \Lambda_{i}$. The even and odd couplings are then naturally

$$
\lambda_{ \pm}=\lambda_{1} \pm \lambda_{2} .
$$

Coordinates on the critical surface should correspond to moduli of the background. One of these, the string coupling and axion, is clearly invariant under the parity symmetry implementing $\mathbf{Z}_{2}$, so maps to the even operator. In the case at hand, the derivation from the quotient singularity of the previous subsection allows us to verify this directly. If we set $\zeta_{2}=\zeta_{3}$, and if further in the quotient model we set all gauge couplings equal $\tau_{i}=\tau=\tau_{S}$, then the model we obtain will be at the $\mathbb{Z}_{2}$ invariant point. Then (6.14) shows that in fact the dimensionless coupling defined above satisfies the one-loop matching condition $\lambda=\Lambda / \zeta_{2}=e^{2 \pi i \tau_{S}}$. The odd coupling should correspond to the periods of $B_{N S}$ and $B_{R R}$ about the vanishing two-cycle. This changes sign under the $\mathbf{Z}_{2}$ (acting on our invariants as $a \rightarrow-a$, it clearly induces the flop).

The type IIB compactification has as always one modulus, invariant under all the symmetries, the string coupling $\tau_{S}$. Above, we showed that the worldvolume coupling $\lambda_{+}$is determined by the asymptotic value of the dilaton in the ambient space. The scaling argument relates this to the string coupling in the dual IIB model. As is clear in the description as a topological product space, $H^{5}$ has one nontrivial two-cycle. The interpretation of the odd coupling, based upon previous experience, is that it reflects the two-form periods about this $S^{2}$. This is the same cycle we identified in the previous discussion (on the six-manifold) and as explained there is indeed $\mathbb{Z}_{2}$-odd. Thus we are led to identify

$$
\begin{aligned}
& \lambda_{+}=e^{2 \pi i \tau_{S}} \\
& \lambda_{-}=f(b),
\end{aligned}
$$


for some function $f$. As before, the periodicity of $b$ predicts $S$-dualities of the conformal field theory; in this case these are the more interesting because we have an interacting fixed point. On the other hand, we do not know the precise form of $f$.

There is a particularly interesting point in the space of deformations of this theory, namely the point at which the two-form periods both vanish. At this point the conformal field theory describing the closed-string sector is singular. The singularity is signalled by the fact that the tension of the wrapped D3-brane strings vanishes. We thus expect a singularity in the worldvolume theory. This point presumably corresponds to the limit in which the gauge couplings are infinite; the $\mathbb{Z}_{2}$ symmetry will be restored at this point.

\subsection{The suspended pinch point singularity}

The toric analysis of section 4 shows that in codimension two in the parameter space, where the $\mathbb{Z}_{2}$ locus intersects the conifold locus, we find a suspended pinch point singularity. We can locate this at $\zeta_{1}=\zeta_{2}=0$. To find the worldvolume theory in this case we choose $\zeta_{3} \gg 0$. The maximally symmetric ground state is then represented by

$$
Z_{34}=\zeta_{3}^{1 / 2}
$$

breaking the gauge symmetry down to $S U(N) \times S U(N) \times S U(N)$. Proceeding as above we find that the light fields and their representations are

$$
\begin{array}{lllllll}
X & =X_{14} & (\mathbf{N}, \mathbf{1}, \overline{\mathbf{N}}) & Y=Y_{24} & (\mathbf{1}, \mathbf{N}, \overline{\mathbf{N}}) & Z=Z_{12} & (\mathbf{N}, \overline{\mathbf{N}}, \mathbf{1}) \\
\tilde{X}=Y_{31} & (\overline{\mathbf{N}}, \mathbf{1}, \mathbf{N}) & \tilde{Y}=X_{32} & (1, \overline{\mathbf{N}}, \mathbf{N}) & \tilde{Z}=Z_{21} & (\overline{\mathbf{N}}, \mathbf{N}, \mathbf{1})
\end{array}
$$

and

$$
\phi=Z_{43} \quad\left(1,1, \mathbf{N}^{2}-1\right) .
$$

Integrating the massive fields out by imposing their equations of motion we find the superpotential

$$
W=\operatorname{tr}\left(\phi(\tilde{Y} Y-\tilde{X} X)+\zeta_{3}^{-1 / 2}(Z \tilde{Z} X \tilde{X}-\tilde{Z} Z \tilde{Y} Y)\right) .
$$

Comparing with the discussion in section four we see that in this case setting $N=1$ will not lead directly to an identical description. Indeed, (6.20) does not vanish in this case.

The model has a $U(1)^{5}$ global symmetry group preserving the superpotential up to an overall phase. Of this, one $U(1)$ is anomalous and combines as usual with the naïve $U(1)_{R}$ to yield the non-anomalous $U(1)_{R}^{\prime}$ under 
which the bifundamental fields have unit charge and $\phi$ charge 2 . Of the non$R$ symmetries, a $U(1)^{2}$ subgroup are baryonic. There are additional discrete symmetries of the model. One of these exchanges the first two factors of the gauge group, simultaneously exchanging $X$ with $Y$ (and $\tilde{X}$ with $\tilde{Y}$ ) and $Z$ with $\tilde{Z}$. This is an $R$-symmetry because the superpotential changes sign and the previous footnotes apply. The other symmetry acts as complex conjugation on the vector multiplets and on the components of $\phi$ while replacing $X^{I}$ with $\tilde{X}^{I}$. This preserves the superpotential. The total global symmetry group is thus

$$
U(1)^{5} \rtimes\left(\mathbb{Z}_{2} \times \mathbb{Z}_{2}\right)
$$

Comparing to (4.35) we see that as expected, the baryonic $U(1)^{2}$ and charge conjugation are not realized as isometries. The latter is the center of the duality group. The former should correspond to periods of the RR four-form about three-cycles in $H^{5}$. Because the horizon in this case is not smooth we will need to resort to our heuristic description of the cycles, justified by its success in describing non-isolated quotients. The construction of section 4 suggests, along these lines, that there are two kinds of threecycles on $H^{5}$. The first, which we denote $A$, arising from the fiber structure as in the case of the conifold, is topologically $S^{3}$. The second, arising as in the non-isolated quotient case, is $C=\Sigma \times S^{1}$ where the $S^{1}$ is the singular circle $x_{0}=y_{0}=0$ and $\Sigma$ is the two-sphere resolving the $\mathbb{C}^{2} / \mathbb{Z}_{2}$ singularity in the transverse space to the circle. This description suggests that one of the $\mathbb{Z}_{2}$ generators in (6.21) be interpreted as the quantum symmetry associated to this singularity. This is the first generator mentioned above; the baryon current associated to $C$ cycle should be reversed by this, identifying this as $J_{1}-J_{2}$, where $J_{i}$ couples to the $U(1)$ in the $i^{\text {th }}$ factor of the gauge group. We complete our basis with the even current $J_{3}$. The basis of three-cycles will be discussed below.

The moduli space of vacua is found once more by constructing the holomorphic invariants in the fields. Once more we find several branches. The invariants are traces of products of

$$
\begin{aligned}
& a=\tilde{X} X=\tilde{Y} Y \quad c=X \tilde{Y} \tilde{Z} \\
& b=\tilde{Z} Z \quad d=Y \tilde{X} Z
\end{aligned}
$$

and

$$
\phi
$$

where the second equality in the first line uses (6.20). Using the rest of $(6.20)$ we see that under traces these all commute. They satisfy the relation

$$
a^{2} b=c d \text {. }
$$

To these we must add baryonic invariants, in this case we have one baryon for each bifundamental chiral multiplet, satisfying the relations

$$
B_{X} B_{\tilde{X}} \sim B_{Y} B_{\tilde{Y}} \sim a^{N}
$$




$$
B_{Z} B_{\tilde{Z}} \sim b^{N} .
$$

The traces of products of (6.22) parameterize a symmetric product of $N$ copies of the space determined by (6.23) . Comparing to the second line of Table 4, we see that this is precisely the suspended pinch point. Thus these moduli describe the motion of $N$ branes in the vicinity of the singularity. At generic points in the space, $\phi$ is massive. The gauge symmetry is broken down to $U(1)^{N-1}$, and all massless matter is neutral, leading to the expected accidental $\mathcal{N}=4$ SUSY. Incorporating the baryonic fields we expect to find as above that the moduli of the singularity itself, as well as the positions of the branes on the resolved geometry, comprise the moduli space.

There is another branch of the moduli space along which $\phi$ is nonvanishing. Then (6.20) implies that of the invariants above only $b$ can be nonzero, while $X$ and $Y$ are massive. This branch thus has the form $\left(\mathbb{C}^{N} / S_{N}\right)^{2}$ and describes the splitting of branes into pairs of fractional branes constrained to move along the $z$ axis, where there is a vanishing cycle. The gauge symmetry is generically $U(1)^{2 N-1}$ and there is no charged massless matter. Along this branch, only $B_{Z}$ and $B_{\tilde{Z}}$ are massless. Along this branch the moment map satisfies $\zeta_{3}=0$. As usual there are mixed branches in which some of the branes have split and $\phi$ has smaller rank.

We can identify the baryons in the $C F T$ with particles in the $A d S$ compactification representing D3-branes wrapping three-cycles in the horizon. The discussion above allows us to identify these. The fact that $B_{Z}$ and $B_{\tilde{Z}}$ remain massless for vacua corresponding to generic $\zeta_{1}$ provided $\zeta_{3}=0$ shows they are related to the three-cycle $C$ from our discussion above. They are thus interpreted as D3-branes wrapped about $\pm C$. Note that these two are indeed exchanged by the quantum symmetry, as one would expect. The cycle $A$, as in the conifold case, has two natural representatives. Recall from section 4 that the horizon $H^{5}$ is a $U(1)$ bundle over $\mathbb{W} \mathbb{C P}^{1,2} \times \mathbb{W} \mathbb{C P}^{1,2}$. Over a point in the first factor in the base we find a three-cycle we can denote $A$. Over a point in the second factor we find a cycle which one can show is homologically $\tilde{A}=-A$. We keep these two distinct since they transform differently under the global symmetry group (again in analogy with the conifold). The quantum symmetry acts on the homology as $C \rightarrow-C$ and $A \rightarrow A-C$, and the charges under the baryonic symmetries show that the baryonic states correspond to D3-branes wrapping three-cycles as follows

$$
\begin{array}{lll}
B_{X} \rightarrow A & B_{Y} \rightarrow A-C & B_{Z} \rightarrow C \\
B_{\tilde{X}} \rightarrow \tilde{A} & B_{\tilde{Y}} \rightarrow \tilde{A}+C & B_{\tilde{Z}} \rightarrow-C .
\end{array}
$$

To look for candidate truly marginal operators we once more appeal to the methods of [115] . The most general gauge-invariant superpotential preserving the full global symmetry has two couplings multiplying the two kinds of terms in (6.20). The anomalous dimensions of $X$ and $Y$ are constrained to 
be the same, while $Z$ can have a different dimension. The scaling coefficients are

$$
\begin{array}{rlr}
A_{g_{1,2}} & =-2 N\left(1+\gamma_{X, Y}+\gamma_{Z}\right) \quad A_{\lambda}=\gamma_{0} / 2+\gamma_{X, Y} \\
A_{g_{3}} & =-2 N\left(\gamma_{0}+2 \gamma_{X, Y}\right) \quad A_{h}=1+\gamma_{Z}+\gamma_{X, Y},
\end{array}
$$

where $\gamma_{0}$ is the dimension of $\phi$. These satisfy two linear relations, so we predict two marginal couplings neutral under all of (6.21) . Relaxing the symmetry conditions and considering also terms breaking the quantum symmetry mentioned above, the four terms in $W$ now have independent couplings, and the scaling coefficients are

$$
\begin{aligned}
& A_{g_{1}}=-2 N\left(1+\gamma_{Z}+\gamma_{X}\right) \\
& A_{g_{2}}=-2 N\left(1+\gamma_{Z}+\gamma_{Y}\right) \\
& A_{g_{3}}=-2 N\left(\gamma_{0}+\gamma_{X}+\gamma_{Y}\right) \\
& A_{\lambda_{1}}=\gamma_{0} / 2+\gamma_{Y} \\
& A_{\lambda_{2}}=\gamma_{0} / 2+\gamma_{X} \\
& A_{h_{1}}=1+\gamma_{Z}+\gamma_{X} \\
& A_{h_{2}}=1+\gamma_{Z}+\gamma_{Y} .
\end{aligned}
$$

The seven functions satisfy three linear relations so that we predict one additional, $\mathbb{Z}_{2}$-odd, marginal coupling.

Interpreting these along the lines of the previous subsection, the one-loop $\beta$-function for $g_{3}$ vanishes, so we can take this as one of the even couplings. The $\beta$-functions for the other two factors do not vanish and as usual the couplings are transmuted into dynamical scales $\Lambda_{1,2}$. Together with the superpotential couplings we can form the dimensionless quantities $\lambda_{i}=h_{i} \Lambda_{i}$. These are permuted by $\mathbb{Z}_{2}$, so lead to one even and one odd coupling. As above, we can directly identify the even coupling by recalling the derivation from the quotient model. One-loop matching yields (working at the $\mathbb{Z}_{2}$ invariant point)

$$
g_{3}=\Lambda / \zeta=e^{2 \pi i \tau_{S}},
$$

so that all three couplings $g_{i}$ are equal to the string coupling. The other two couplings will arise from two-form periods. The odd coupling is clearly associated to the two-cycle resolving the $\mathbb{Z}_{2}$ singularity; the even coupling can be associated to either of the two factors in the base.

\subsection{The complex cone over $\mathbb{F}_{0}$}

We can apply the methods here to study one more singularity. This is described as a $\mathbb{Z}_{2}$ quotient of the conifold singularity, the action on the 
coordinates of $(6.11)$ being $(a, b, c, d) \rightarrow(-a,-b,-c,-d)$. We can realize this model with our techniques because it can be described as a $\mathbb{Z}_{2}$ quotient of the conifold as mentioned earlier. We thus apply once more the methods of [102] for dealing with quotients. To study $N$ branes at this point we need to study $2 N$ branes at the conifold, a theory we have discussed above. We take the $\mathbb{Z}_{2}$ action on the fields of (6.6) to be $X \rightarrow-X$. Note that on the invariants of (6.10) this acts by reversing all signs, preserving (6.11) and fixing only the origin.

The gauge fields surviving the projection correspond to the subgroup $S U(N)^{4}$. The surviving matter multiplets and their charges are

$$
\begin{array}{llll}
X_{i 12} & (\mathbf{N}, \mathbf{1}, \mathbf{1}, \overline{\mathbf{N}}) & Y_{i 22} & (1, \overline{\mathbf{N}}, \mathbf{1}, \mathbf{N}), \\
X_{i 21} & (\mathbf{1}, \mathbf{N}, \overline{\mathbf{N}}, \mathbf{1}) & Y_{i 22} & (\mathbf{1}, \overline{\mathbf{N}}, \mathbf{1}, \mathbf{N})
\end{array}
$$

and the superpotential takes the form

$$
W=\operatorname{tr}\left(\epsilon^{i j} \epsilon^{k l} X_{i 12} Y_{k 22} X_{j 21} Y_{l 11}\right)
$$

This leaves unbroken a $U(2) \times U(2) \times U(1) \times U(1)$ global symmetry. The $U(2)^{2}$ acts as in (6.8). The diagonal $U(1)$ subgroup of this is anomalous, and as usual combines with the naivve $R$-symmetry to form the non-anomalous $U(1)_{R}^{\prime}$ under which all lowest components of the chiral fields have charge $1 / 2$. The remaining $U(1)^{3}$ are the baryon number symmetries. Note that in this chiral theory we find that in fact two of these are in fact broken by gauge anomalies, so that only one baryon number symmetry survives. In addition, there is a discrete $\mathbb{Z}_{4}$ symmetry which permutes the factors in the gauge group according to the cycle (1423) and permutes the four types of chiral multiplets cyclically so that the trace in (6.30) is unchanged. Including action on the vector multiplets by charge conjugation, together with a permutation, say (12) so that the representation content is preserved, and an action on the matter multiplets by the permutation determined by the representations (in the case at hand this is $X_{12} \leftrightarrow Y_{22}$ and $X_{21} \leftrightarrow Y_{11}$ ) extends this to an entire $D_{4}$ group. Two of the elements of order two are associated to charge conjugation. The third, the square of the order four element, is in fact the quantum symmetry associated to the $\mathbb{Z}_{2}$ quotient we used to construct the model from the conifold. Note that two of the order four elements (the ones involving charge conjugation) do not preserve the superpotential and must be accompanied by appropriate $U(1)_{R}$ rotations. The correct global symmetry group is thus

$$
U(2) \times U(2) \rtimes D_{4} .
$$

Comparing to (4.29) we find the expected differences. The non-anomalous baryon number symmetry corresponds to the gauge symmetry carried by the period of the RR four-form about the unique (non-torsion) three-cycle on the 
horizon. The $\mathbb{Z}_{2}$ isometry found in section 4 squares in fact to the quantum symmetry, generating the $\mathbb{Z}_{4}$ discrete symmetry. The additional $\mathbb{Z}_{2}$ action associated to charge conjugation corresponds to the center of $S L(2, \mathbb{Z})$.

The classical moduli space of vacua for this theory is parameterized by the invariant traces. In this case the generators are in fact quartic expressions, of the form

$$
c_{i j k l}=X_{i 12} Y_{j 22} X_{k 21} Y_{l 11} .
$$

The equations of motion following from (6.30) show that these satisfy

$$
\begin{aligned}
c_{i j k l} & =c_{k j i l}=c_{i l k j} \\
c_{i j k l} c_{m n r s} & =c_{i j r s} c_{m n k l},
\end{aligned}
$$

so that they can be written as quadratic expressions $c_{i j k l}=a_{i j} a_{k l}$ with the $a_{i j}$ satisfying (6.11). This corresponds to our representation of the transverse space as a $\mathbb{Z}_{2}$ quotient of the conifold. To these we must add the baryonic invariants

$$
\begin{aligned}
B_{s 12} & \sim X_{12}^{s} X_{212}^{N-s} & B_{s 11} & \sim Y_{111}^{s} Y_{211}^{N-s} \\
B_{s 21} & \sim X_{121}^{s} X_{221}^{N-s} & B_{s 22} & \sim Y_{122}^{s} Y_{22}^{N-s} .
\end{aligned}
$$

These transform in two copies of $(\mathbf{N}+1,1)_{N} \oplus(\mathbf{1}, \mathbf{N}+\mathbf{1})_{-N}$ under the continuous global symmetry (we label the representation by $S U(2)^{2} \times U(1)_{B}$ content; all these state have $U(1)_{R}^{\prime}$ charge $\left.N / 2\right)$. Their transformation properties under the discrete group follow from those of the chiral multiplets (6.29) . They satisfy relations of which a representative is

$$
B_{s 12} B_{r 22} B_{t 21} B_{u 11} \sim c_{1111}^{u} c_{1112}^{t-u} c_{1122}^{r-t} c_{1222}^{s-r} c_{2222}^{N-s},
$$

valid when $s \geq r \geq t \geq u$ and $s-r \leq r-t$.

As in the previous cases, the meson-like degrees of freedom (6.32) parameterize the $N^{\text {th }}$ symmetric product of the singularity in question. Incorporating the baryons, we expect to find the moduli of the singularity as well. At generic points on this branch the gauge symmetry is $U(1)^{N-1}$; the $\mathcal{N}=4$ supersymmetry is restored, as expected.

However, there is a subtlety in this case that was absent in the previous ones. There are additional branches in the moduli space which are purely baryonic, hence absent from the description we have given. To see these, note that the symmetries of $c_{i j k l}$ show that (6.35) depends on the $U(2)$ indices only through the combinations $s+t$ and $u+r$. In fact, (6.35) together with (6.33) are generically sufficient to yield

$$
B_{s 12}=B_{s 21} \quad \text { and } \quad B_{s 11}=B_{s 22} .
$$

If we now consider regions of moduli space in which all the $Y$ variables vanish, however, we find that $c_{i j k l}=B_{11}=B_{22}=0$, and the remaining baryons are 
subject to no such restriction. Thus, in this region, new baryonic degrees of freedom are light and can parameterize new branches of the moduli space. To understand the low-energy physics along these branches we return to the description in terms of the charged fields, and assign expectation values to $X_{112}$ and $X_{121}$. We then see that the gauge group is broken to $S U(N) \times$ $S U(N)$, and the light matter surviving the Higgs mechanism is precisely that of an $\mathcal{N}=2$ theory with this gauge group and two hypermultiplets in the $(\mathbf{N}, \overline{\mathbf{N}})$ representation; inserting the expectation values into (6.30) leaves the expected cubic superpotential. The baryonic moduli in the full theory map out the Coulomb branch of the low-energy theory. The Higgs branch of the latter is a subspace of the large branch discussed above. The couplings in the $\mathcal{N}=2$ model are determined by the remaining baryonic moduli. There is of course a similar story when the $X$ variables vanish. Since the new branches are baryonic we cannot form mixed branches.

This description of new branches is consistent with the geometry described in section 4. Recall that for each of the partial resolutions of this singularity there is a curve of $\mathbb{Z}_{2}$ quotient singularities. In this situation we would expect "fractional" branches in which wrapped D5-branes are confined to the singular curve, in agreement with the description we have given.

We now turn to the counting of marginal operators in the theory. This is simplified in this case by the fact that (6.30) is the unique superpotential preserving the continuous global symmetry. Thus we have

$$
\begin{aligned}
& A_{g_{1}}=-2 N\left(1+\gamma_{12}+\gamma_{11}\right) \\
& A_{g_{2}}=-2 N\left(1+\gamma_{21}+\gamma_{22}\right) \\
& A_{g_{3}}=-2 N\left(1+\gamma_{21}+\gamma_{11}\right) \\
& A_{g_{4}}=-2 N\left(1+\gamma_{12}+\gamma_{22}\right) \\
& A_{h}=1+\frac{1}{2}\left(\gamma_{12}+\gamma_{22}+\gamma_{21}+\gamma_{11}\right) .
\end{aligned}
$$

These are easily seen to obey two linear relations, leading to a two-dimensional space of fixed points. As usual, one of these, preserving the full discrete symmetry, corresponds to the string coupling, while the other, transforming by a sign under all order four elements of the discrete group, should be associated to two-form periods. The marginal operators in the $A d S$ description will be the IIB string coupling as well as the two-form periods. The relevant cohomology group in this case is $H_{\mathrm{DR}}^{2}\left(H^{5}\right)=\mathbb{Z}$ so we indeed expect precisely one such coupling.

We can also compare the baryon spectrum to the homology cycles on $H^{5}$ and the natural parameter spaces for their representatives. As discussed in section 4 , the relevant homology group $H_{3}\left(H^{5}\right)$ is $\mathbb{Z} \oplus \mathbb{Z}_{2}$. The natural representative cycles are two families of three-spheres, such that their sum is the torsion element. The parameter space for each of these is a two-sphere. 
This is in agreement with the discussion above. In the "large" branch of the moduli space, there are indeed two flavors of baryons, since $B_{12} \sim B_{21}$ and $B_{11} \sim B_{22}$. These have opposite charge under the baryonic $U(1)$ symmetry, so that a state with two baryons, one from each family, is neutral. They also transform in the spin- $N / 2$ representation of (distinct) $S U(2)$ global symmetry groups, corresponding to the quantization of collective coordinates on the two-spheres.

\section{Discussion}

Extending the $A d S / C F T$ correspondence to describe branes at singularities is, as we have stressed, required for a complete description of the duality. Since configurations with branes at singularities occur at a finite distance in moduli space, the modifications to the extreme low-energy theory at these points should be captured by an appropriate modification of the $A d S$ compactification. We have argued that the modification in question is to replace the spherical horizon with a (non-spherical) horizon manifold determined by the local geometry at the singular point. We expect this statement to hold quite generally for the types of singularities encountered at finite distance. In this paper, we have offered some evidence to support this extension of the conjecture. In complete generality, we find that the $R$-symmetry group of the CFT is realized in the dual model by isometries of the horizon. This correspondence is predicted by the conjectures and the fact that it obtains is very encouraging. Further evidence is found by considering individual models in detail.

We have presented such detailed studies of a small set of examples in the D3-brane case. (It will be interesting to extend this work to the case of M5-branes [11] as well as to the more challenging case of M2-branes.) Our approach to these D3-brane models allows us to derive the worldvolume Lagrangian (including interactions) from an explicit construction. In each case we have studied, comparisons with the predictions of the dual AdS model give additional evidence for the extended conjecture. The agreement in the baryon spectrum, in particular, is noteworthy. These states are chiral primaries, but their conformal weight grows at large $N$ like $N$; most tests of the conjectures thus far were restricted to operators whose weight remains finite in this limit. As in $[107,32]$ we interpret this agreement as evidence that finite $N$ effects are captured as stringy corrections to the SUGRA theory on $A d S$. Our discussion of these states has necessitated a careful study of the fate of Abelian factors in the gauge group. Extending our observations on orbifold models, we have formulated a conjecture according to which these behave in one of two ways, depending upon the nature of the blowup to which they correspond ("small" or "big"). An explicit computation supporting this, giving evidence from additional examples, is expected to clarify this 
issue soon. Another feature of our approach is that we directly find relations between the various models by embedding them all in the original orbifold theory. Thus, we can describe deformations of one into the other quite explicitly.

Our discussion of these examples, while encouraging, has not been complete. Most dramatically, we have not discussed the most interesting singularitiesthe points in the moduli space at which the two-form periods vanish. At these points, the conformal field theory describing the closed-string perturbation theory is singular, and we expect new light degrees of freedom, described by wrapped branes, to propagate in the brane worldvolume. It is not clear if these will decouple from the low-energy theory on the worldvolume but this appears unlikely. A more explicit description of these theories than the one we have obtained - as the infinite-coupling limit of one of our gauge theories-would presumably lead to an understanding of this issue.

Related to this is the question of the quantum corrections to the classical moduli spaces we have constructed. These are important near the interacting conformal theories and understanding the corrections in the dual $A d S$ model would be useful. More speculatively, it should be possible to make more explicit statements about the UV/IR connection that has played such an important role in our construction. In particular, some properties of RG flow should find corresponding properties in the radial dependence of the background fields in the associated SUGRA solutions.

\section{Acknowledgements}

We would like to thank Eva Silverstein for collaboration in the early stages of this project. We would also like to thank Ofer Aharony, Paul Aspinwall, Robert Bryant, Brian Greene, Jeff Harvey, Juan Maldacena, Greg Moore, Bill Pardon, and Nick Warner for discussions. We gratefully acknowledge the hospitality and support of the Institute for Theoretical Physics, Santa Barbara (D.R.M.) and the Aspen Center for Physics (D.R.M. and M.R.P.). The work of D.R.M. was supported in part by NSF grant DMS-9401447, and the work of M.R.P. was supported in part by the Israel Academy of Sciences and Humanities Centres of Excellence Programme.

\section{Appendix A. Sasaki and $G_{2}$ structures}

We collect here the definitions of the various geometric structures which occur on horizon manifolds, taken from [49, 50,44]. All of these are implied by existence of certain numbers of Killing spinors, as we explained in section 2 . 
A metric contact structure on a Riemannian manifold $H^{2 n-1}$ (with metric $\langle\rangle$,$) consists of a vector field X$, a one-form $\eta$, and a $(1,1)$ tensor field $\phi$ such that

(i) $\eta \wedge(d \eta)^{n-1} \neq 0$

(ii) $\eta(X)=1, \phi(X)=0$ (iii) $\phi^{2}=-\mathbb{1}+\eta \otimes X$

(iv) $\langle\phi(V), \phi(W)\rangle=\langle V, W\rangle-\eta(V) \eta(W)$

(v) $d \eta(V, W)=2\langle V, \phi(W)\rangle$ where $d \eta(V, W)=V(\eta(W))-W(\eta(V))-\eta([V, W])$.

A Sasaki structure is a metric contact structure such that

(vi) $X$ is a Killing vector field, or equivalently, $\nabla_{V} X=-\phi(V)$

(vii) $\left(\nabla_{V} \phi\right)(W)=\langle V, W\rangle X-\eta(W) V$.

A Kähler structure on the cone $C(H)$ determines a Sasaki structure on $H$ as follows. Let $J$ be the parallel complex structure on $C(H)$ determined by the Kähler structure. Then

$$
X=J\left(\partial_{r}\right), \quad \eta(V)=\langle X, V\rangle, \quad \phi=\nabla X
$$

defines the Sasaki structure, where $\partial_{r}$ is the radial vector field.

A 3-Sasaki structure ${ }^{29}$ consists of a triple of Sasaki structures $\left(\phi_{i}, X_{i}, \eta_{i}\right)$ such that

(i) $X_{1}, X_{2}, X_{3}$ are orthonormal

(ii) $\left[X_{1}, X_{2}\right]=2 X_{3},\left[X_{2}, X_{3}\right]=2 X_{1},\left[X_{3}, X_{1}\right]=2 X_{2}$

(iii) $\phi_{3} \phi_{2}=-\phi_{1}+\eta_{2} \otimes X_{3}, \quad \phi_{2} \phi_{3}=\phi_{1}+\eta_{3} \otimes X_{2}$,

$\phi_{1} \phi_{3}=-\phi_{2}+\eta_{3} \otimes X_{1}, \quad \phi_{3} \phi_{1}=\phi_{2}+\eta_{1} \otimes X_{3}$,

$\phi_{2} \phi_{1}=-\phi_{3}+\eta_{1} \otimes X_{2}, \quad \phi_{1} \phi_{2}=\phi_{3}+\eta_{2} \otimes X_{1}$.

A hyper-Kähler structure on the cone $C(H)$ determines a 3-Sasaki structure on $H$ as follows. Let $I, J, K$ be parallel complex structures on $C(H)$ such that $I J=-J I=K$. Then the three Sasaki structures are given by

$$
X_{1}=I\left(\partial_{r}\right), \quad X_{2}=J\left(\partial_{r}\right), \quad X_{3}=-K\left(\partial_{r}\right)
$$

with $\eta_{i}$ and $\phi_{i}$ determined from $X_{i}$ as in (A.1).

To describe nearly-parallel $G_{2}$-structures, we must first recall that the action of $S O(7)$ on $\Lambda^{3}\left(\mathbb{R}^{7}\right)^{*}$ has two open orbits, the definite three-forms and the indefinite three-forms [46]; the stabilizer of any definite three-form is (conjugate to) the compact group $G_{2}$ whereas the stabilizer of an indefinite three-form is the non-compact form of $G_{2}$. A three-form on a seven-manifold is called definite if it is definite in each tangent space.

A nearly-parallel $G_{2}$-manifold ${ }^{30}$ is a seven-manifold with a definite threeform $\phi$ such that $\nabla \phi=\star \phi$. The Riemannian metric is automatically Einstein. There is a one-to-one correspondence between nearly-parallel $G_{2^{-}}$ structures on $H$ and $\operatorname{Spin}(7)$-structures on $C(H)$.

\footnotetext{
${ }^{29}$ These structures have recently made an appearance in the study of rigid $\mathcal{N}=2$ superconformal hypermultiplets [119].

${ }^{30}$ We thank K. Galicki for a discussion on terminology, and for pointing us to his expository paper with C. P. Boyer (hep-th/9810250) which contains an extensive bibliography on this subject.
} 


\section{Appendix B. $D$-term equations and Moduli Spaces}

The standard construction of the moduli space for the $\mathbb{Z}_{2} \times \mathbb{Z}_{2}$ quotient proceeds by constructing all the holomorphic $S U(N)^{4}$ invariants in the chiral fields, modulo the $F$-term relations. In addition to (5.15) we then have the baryons $B_{i j} \sim X_{i j}^{N}$, subject to relations of the form (suppressing numerical factors)

$$
\begin{array}{lll}
B_{14} B_{41}=x_{1}^{N} & B_{13} B_{31}=y_{1}^{N} & B_{12} B_{21}=z_{1}^{N} \\
B_{23} B_{32}=x_{2}^{N} & B_{24} B_{42}=y_{2}^{N} & B_{34} B_{43}=z_{2}^{N}
\end{array}
$$

Together with (5.15) these give coordinates on the full moduli space.

The $D$-term equations for this case are

$$
\begin{aligned}
& X_{14} X_{14}^{\dagger}+Y_{13} Y_{13}^{\dagger}+Z_{12} Z_{12}^{\dagger}-X_{41}^{\dagger} X_{41}-Y_{31}^{\dagger} Y_{31}-Z_{21}^{\dagger} Z_{21}=\zeta_{1} \\
& X_{23} X_{23}^{\dagger}+Y_{24} Y_{24}^{\dagger}+Z_{21} Z_{21}^{\dagger}-X_{32}^{\dagger} X_{32}-Y_{42}^{\dagger} Y_{42}-Z_{12}^{\dagger} Z_{12}=\zeta_{2} \\
& X_{32} X_{32}^{\dagger}+Y_{31} Y_{31}^{\dagger}+Z_{34} Z_{34}^{\dagger}-X_{23}^{\dagger} X_{23}-Y_{13}^{\dagger} Y_{13}-Z_{43}^{\dagger} Z_{43}=\zeta_{3} \\
& X_{41} X_{41}^{\dagger}+Y_{42} Y_{42}^{\dagger}+Z_{43} Z_{43}^{\dagger}-X_{14}^{\dagger} X_{14}-Y_{24}^{\dagger} Y_{24}-Z_{34}^{\dagger} Z_{34}=\zeta_{4},
\end{aligned}
$$

where the $\zeta_{i}$ are free to vary subject to $\sum_{i} \zeta_{i}=0$. As in section 5 , it is useful to study the various "slices" of this moduli space in which the $\zeta_{i}$ 's are held constant.

To make contact with the description of this space in terms of toric geometry, and in particular to correctly identify the points in moduli space corresponding to interesting singular geometries, we review from [102, 103, 104] another description of this space in the case $N=1 .{ }^{31}$ In that case, the $F$-flatness conditions arising from (5.13) themselves describe a toric variety, as they are all of the form "one monomial equals another monomial." Some combinatorial manipulations ([103, 104], following the method of [112]) show that we can describe this toric variety as a quotient of $\mathbb{C}^{9}$ with (homogeneous) coordinates $p_{1}, \ldots, p_{9}$ by $U(1)^{3}$, acting with charge matrix: ${ }^{32}$

$$
\left(\begin{array}{ccccccccc}
p_{1} & p_{2} & p_{3} & p_{4} & p_{5} & p_{6} & p_{7} & p_{8} & p_{9} \\
0 & -1 & -1 & 1 & 0 & 0 & 1 & 0 & 0 \\
-1 & 0 & -1 & 0 & 1 & 0 & 0 & 1 & 0 \\
-1 & -1 & 0 & 0 & 0 & 1 & 0 & 0 & 1
\end{array}\right)
$$

The invariant coordinates are:

$$
\begin{array}{lllll}
X_{14}=p_{1} p_{8} p_{9} & X_{23}=p_{1} p_{5} p_{9} & X_{32}=p_{1} p_{6} p_{8} & X_{41}=p_{1} p_{5} p_{6} \\
Y_{13}=p_{2} p_{4} p_{9} & Y_{24}=p_{2} p_{7} p_{9} & Y_{31}=p_{2} p_{6} p_{7} & Y_{42}=p_{2} p_{4} p_{6} \\
Z_{12}=p_{3} p_{4} p_{8} & Z_{21}=p_{3} p_{5} p_{7} & Z_{34}=p_{3} p_{7} p_{8} & Z_{43}=p_{3} p_{4} p_{5}
\end{array}
$$

\footnotetext{
${ }^{31}$ This description does not appear to directly generalize past $N=1$.

${ }^{32}$ We have used different bases than either [103] or [104], in order to clarify certain points below.
} 
These satisfy all of the $F$-term equations, viz.,

$$
\begin{array}{cl}
X_{14} Y_{42}=p_{1} p_{2} p_{4} p_{6} p_{8} p_{9}=Y_{13} X_{32}, & X_{14} Z_{43}=p_{1} p_{3} p_{4} p_{5} p_{8} p_{9}=Z_{12} X_{23} \\
X_{23} Y_{31}=p_{1} p_{2} p_{5} p_{6} p_{7} p_{9}=Y_{24} X_{41}, & X_{23} Z_{34}=p_{1} p_{3} p_{5} p_{7} p_{8} p_{9}=Z_{21} X_{14} \\
X_{32} Y_{24}=p_{1} p_{2} p_{6} p_{7} p_{8} p_{9}=Y_{31} X_{14}, & X_{32} Z_{21}=p_{1} p_{3} p_{5} p_{6} p_{7} p_{8}=Z_{34} X_{41} \\
X_{41} Y_{13}=p_{1} p_{2} p_{4} p_{5} p_{6} p_{9}=Y_{42} X_{23}, & X_{41} Z_{12}=p_{1} p_{3} p_{4} p_{5} p_{6} p_{8}=Z_{43} X_{32} \\
Y_{13} Z_{34}=p_{2} p_{3} p_{4} p_{7} p_{8} p_{9}=Z_{12} Y_{24} \\
Y_{24} Z_{43}=p_{2} p_{3} p_{4} p_{5} p_{7} p_{9}=Z_{21} Y_{13} \\
Y_{31} Z_{12}=p_{2} p_{3} p_{4} p_{6} p_{7} p_{8}=Z_{34} Y_{42} \\
Y_{42} Z_{21}=p_{2} p_{3} p_{4} p_{5} p_{6} p_{7}=Z_{43} Y_{31} .
\end{array}
$$

The $D$-term equations from our theory are associated to a $U(1)^{4}$ action on the fields $X_{i j}, Y_{i j}, Z_{i j}$, and this can be lifted to a second $U(1)^{4}$ action on the fields $p_{\alpha}$, with charge matrix:

$$
\left(\begin{array}{ccccccccc}
p_{1} & p_{2} & p_{3} & p_{4} & p_{5} & p_{6} & p_{7} & p_{8} & p_{9} \\
1 & 0 & 0 & 1 & -1 & -1 & 0 & 0 & 0 \\
0 & 1 & 0 & -1 & 1 & -1 & 0 & 0 & 0 \\
0 & 0 & 1 & -1 & -1 & 1 & 0 & 0 & 0 \\
-1 & -1 & -1 & 1 & 1 & 1 & 0 & 0 & 0
\end{array}\right)
$$

(Of course, the diagonal $U(1)$ acts trivially as expected.) To verify that we have lifted the charge assignments correctly, we calculate the induced charges on the invariant coordinates, from (B.4) and (B.6):

$$
\left(\begin{array}{cccccccccccc}
X_{14} & X_{23} & X_{32} & X_{41} & Y_{13} & Y_{24} & Y_{31} & Y_{42} & Z_{12} & Z_{21} & Z_{34} & Z_{43} \\
1 & 0 & 0 & -1 & 1 & 0 & -1 & 0 & 1 & -1 & 0 & 0 \\
0 & 1 & -1 & 0 & 0 & 1 & 0 & -1 & -1 & 1 & 0 & 0 \\
0 & -1 & 1 & 0 & -1 & 0 & 1 & 0 & 0 & 0 & 1 & -1 \\
-1 & 0 & 0 & 1 & 0 & -1 & 0 & 1 & 0 & 0 & -1 & 1
\end{array}\right)
$$

These are exactly the expected charge assignments for our theory.

The four $U(1)$ 's in (B.6) have associated FI terms with coefficients $\zeta_{1}$, $\zeta_{2}, \zeta_{3}, \zeta_{4}$. If we collect the charges from all seven $U(1)$ 's into a single matrix, and include the FI coefficients as an extra column, we obtain

$$
\left(\begin{array}{cccccccccc}
1 & 0 & 0 & 1 & -1 & -1 & 0 & 0 & 0 & \zeta_{1} \\
0 & 1 & 0 & -1 & 1 & -1 & 0 & 0 & 0 & \zeta_{2} \\
0 & 0 & 1 & -1 & -1 & 1 & 0 & 0 & 0 & \zeta_{3} \\
-1 & -1 & -1 & 1 & 1 & 1 & 0 & 0 & 0 & \zeta_{4} \\
0 & -1 & -1 & 1 & 0 & 0 & 1 & 0 & 0 & 0 \\
-1 & 0 & -1 & 0 & 1 & 0 & 0 & 1 & 0 & 0 \\
-1 & -1 & 0 & 0 & 0 & 1 & 0 & 0 & 1 & 0
\end{array}\right)
$$


Doing a few row operations, this becomes:

$$
\left(\begin{array}{cccccccccc}
1 & 0 & 0 & 1 & -1 & -1 & 0 & 0 & 0 & \zeta_{1} \\
0 & 1 & 0 & -1 & 1 & -1 & 0 & 0 & 0 & \zeta_{2} \\
0 & 0 & 1 & -1 & -1 & 1 & 0 & 0 & 0 & \zeta_{3} \\
0 & 0 & 0 & 0 & 0 & 0 & 0 & 0 & 0 & \sum \zeta_{i} \\
0 & 0 & 0 & -1 & 0 & 0 & 1 & 0 & 0 & \zeta_{2}+\zeta_{3} \\
0 & 0 & 0 & 0 & -1 & 0 & 0 & 1 & 0 & \zeta_{1}+\zeta_{3} \\
0 & 0 & 0 & 0 & 0 & -1 & 0 & 0 & 1 & \zeta_{1}+\zeta_{2}
\end{array}\right) .
$$

(Notice that as expected we must have $\sum \zeta_{i}=0$.) In an appropriate region of $\zeta$ space, we can use the last three rows of (B.9) to eliminate $p_{7}, p_{8}, p_{9}$, and we are left with precisely the toric description of a $\mathbb{Z}_{2} \times \mathbb{Z}_{2}$ quotient singularity given in section 4.2 , with the $D$-terms precisely identified. (The notational coincidence of using $\zeta_{i}$ for the $D$-terms (B.2) is now seen to be a precise correspondence with the moment map of section 4.2.) 


\section{References}

[1] M. J. Duff, R. Minasian, and E. Witten, "Evidence for heterotic/heterotic duality," Nucl. Phys. B 465 (1996) 413-438, hep-th/ 9601036.

[2] O. J. Ganor and A. Hanany, "Small $E_{8}$ instantons and tensionless non critical strings," Nucl. Phys. B 474 (1996) 122-140, hep-th/9602120.

[3] N. Seiberg and E. Witten, "Comments on string dynamics in six dimensions," Nucl. Phys. B 471 (1996) 121-134, hep-th/9603003.

[4] S. Sethi, C. Vafa, and E. Witten, "Constraints on low-dimensional string compactifications," Nucl. Phys. B 480 (1996) 213-224, hep-th/ 9606122 .

[5] J. Maldacena, "The large $\mathrm{N}$ limit of superconformal field theories and supergravity," Adv. Theor. Math. Phys. 2 (1998) 231-252, hep-th/ 9711200.

[6] S. S. Gubser, I. R. Klebanov, and A. M. Polyakov, "Gauge theory correlators from non-critical string theory," Phys. Lett. B 428 (1998) 105-114, hep-th/9802109.

[7] E. Witten, "Anti-de Sitter space and holography," Adv. Theor. Math. Phys. 2 (1998) 253-291, hep-th/9802150.

[8] A. Fayyazuddin and M. Spaliński, "Large N superconformal gauge theories and supergravity orientifolds," Nucl. Phys. B 535 (1998) 219232 , hep-th/9805096.

[9] O. Aharony, A. Fayyazuddin, and J. Maldacena, "The large $\mathrm{N}$ limit of $\mathcal{N}=2,1$ field theories from threebranes in F-theory," J. High Energy Phys. 07 (1998) 013, hep-th/9806159.

[10] C. Ahn, K. Oh, and R. Tatar, "The large $N$ limit of $\mathcal{N}=1$ field theories from F theory," Mod. Phys. Lett. A 14 (1999) 369-378, hep-th/ 9808143.

[11] D. R. Morrison and M. R. Plesser, "Non-spherical horizons, II," in preparation.

[12] S. Kachru and E. Silverstein, "4d conformal field theory and strings on orbifolds," Phys. Rev. Lett. 80 (1998) 4855-4858, hep-th/9802183.

[13] M. Berkooz, "A supergravity dual of a $(1,0)$ field theory in six dimensions," Phys. Lett. B 437 (1998) 315-317, hep-th/9802195. 
[14] A. Lawrence, N. Nekrasov, and C. Vafa, "On conformal field theories in four dimensions," Nucl. Phys. B 532 (1998) 199-209, hep-th/ 9803015.

[15] M. Bershadsky, Z. Kakushadze, and C. Vafa, "String expansion as large $N$ expansion of gauge theories," Nucl. Phys. B 523 (1998) 5972 , hep-th/9803076.

[16] S. Ferrara, A. Kehagias, H. Partouche, and A. Zaffaroni, "Membranes and fivebranes with lower supersymmetry and their AdS supergravity duals," Phys. Lett. B 431 (1998) 42-48, hep-th/9803109.

[17] J. Gomis, "Anti de Sitter geometry and strongly coupled gauge theories," Phys. Lett. B 435 (1998) 299-302, hep-th/9803119.

[18] Y. Oz and J. Terning, "Orbifolds of $A d S_{5} \times S^{5}$ and $4 d$ conformal field theories," Nucl. Phys. B 532 (1998) 163-180, hep-th/9803167.

[19] E. Halyo, "Supergravity on $A d S_{5 / 4} \times$ Hopf fibrations and conformal field theories," hep-th/9803193.

[20] M. Bershadsky and A. Johansen, "Large N limit of orbifold field theories," Nucl. Phys. B 536 (1998) 141-148, hep-th/9803249.

[21] C. Ahn, K. Oh, and R. Tatar, "Orbifolds of $A d S_{7} \times S^{4}$ and six dimensional (0,1) SCFT," Phys. Lett. B 442 (1998) 109-116, hep-th/ 9804093.

[22] C. Ahn, K. Oh, and R. Tatar, "Branes, orbifolds and the three dimensional $\mathcal{N}=2$ SCFT in the large $N$ limit," J. High Energy Phys. 11 (1998) 024, hep-th/9806041.

[23] S. Gukov, "Comments on $\mathcal{N}=2$ AdS orbifolds," Phys. Lett. B. 439 (1998) 23-26, hep-th/9806180.

[24] A. Hanany, M. J. Strassler, and A. M. Uranga, "Finite theories and marginal operators on the brane," J. High Energy Phys. 06 (1998) 011, hep-th/9803086.

[25] A. Hanany and A. M. Uranga, "Brane boxes and branes on singularities," J. High Energy Phys. 05 (1998) 013, hep-th/9805139.

[26] L. Castellani, A. Ceresole, R. D'Auria, S. Ferrara, P. Fré, and M. Trigiante, " $G / H$ M-branes and $A d S_{p+2}$ geometries," Nucl. Phys. B 527 (1998) 142-170, hep-th/9803039.

[27] M. J. Duff, H. Lü, and C. N. Pope, "AdS $5 \times S^{5}$ untwisted," Nucl. Phys. B 532 (1998) 181-209, hep-th/9803061. 
[28] M. J. Duff, H. Lü, C. N. Pope, and E. Sezgin, "Supermembranes with fewer supersymmetries," Phys. Lett. B 371 (1996) 206-214, hep-th/ 9511162.

[29] A. Kehagias, "New type IIB vacua and their F-theory interpretation," Phys. Lett. B 435 (1998) 337-342, hep-th/9805131.

[30] I. R. Klebanov and E. Witten, "Superconformal field theory on threebranes at a Calabi-Yau singularity," Nucl. Phys. B 536 (1998) 199218 , hep-th/9807080.

[31] S. S. Gubser, "Einstein manifolds and conformal field theories," Phys. Rev. D 59 (1999) 025006, hep-th/9807164.

[32] S. S. Gubser and I. R. Klebanov, "Baryons and domain walls in an $\mathcal{N}=1$ superconformal gauge theory," Phys. Rev. D 58 (1998) 125025 hep-th/9808075.

[33] J. M. Figueroa-O'Farrill, "Near-horizon geometries of supersymmetric branes," Talk presented at SUSY '98, hep-th/9807149.

[34] B. S. Acharya, J. M. Figueroa-O'Farrill, C. M. Hull, and B. Spence, "Branes at conical singularities and holography," Adv. Theor. Math. Phys. 2 (1998) 1249-1286, hep-th/9808014.

[35] P. Hořava and E. Witten, "Heterotic and type I string dynamics from eleven dimensions," Nucl. Phys. B 460 (1996) 506-524, hep-th/ 9510209 .

[36] E. Witten, "Phase transitions in $M$-theory and $F$-theory," Nucl. Phys. B 471 (1996) 195-216, hep-th/9603150.

[37] D. R. Morrison and C. Vafa, "Compactifications of F-theory on CalabiYau threefolds, II," Nucl. Phys. B 476 (1996) 437-469, hep-th/ 9603161.

[38] Y. Hayakawa, "Degeneration of Calabi-Yau manifold with WeilPetersson metric," alg-geom/9507016.

[39] C.-L. Wang, "On the incompletenes of the Weil-Petersson metric along degenerations of Calabi-Yau manifolds," Math. Res. Lett. 4 (1997) 157-171.

[40] K. Becker and M. Becker, "M-theory on eight-manifolds," Nucl. Phys. B 477 (1996) 155-167, hep-th/9605053.

[41] G. Horowitz and A. Strominger, "Black strings and p-branes," Nucl. Phys. B 360 (1991) 197-209.

[42] M. J. Duff and K. S. Stelle, "Multimembrane solutions of $D=11$ supergravity," Phys. Lett. B 253 (1991) 113-118. 
[43] R. Güven, "Black p-brane solutions of $D=11$ supergravity," Phys. Lett. B 276 (1992) 49-55.

[44] C. Bär, "Real Killing spinors and holonomy," Comm. Math. Phys. 154 (1993) 509-521.

[45] M. Berger, "Sur les groups d'holonomie des variétés à connexion affine et des variétés riemanniennes," Bull. Soc. Math. France 83 (1955) 279 330 .

[46] R. Bryant, "Metrics with exceptional holonomy," Ann. of Math. (2) 126 (1987) 525-576.

[47] W. Nahm, "Supersymmetries and their representations," Nucl. Phys. B 135 (1978) 149-166.

[48] S. Gallot, "Équations différentielles caractéristiques de la sphère," Ann. Sci. École Norm. Sup. (4) 12 (1979) 235-267.

[49] Th. Friedrich and I. Kath, "Einstein manifolds of dimension five with small first eigenvalue of the Dirac operator," J. Differential Geom. 29 (1989) 263-279.

[50] Th. Friedrich and I. Kath, "Real Killing spinors and holonomy," Comm. Math. Phys. 133 (1990) 543-561.

[51] C. P. Boyer, K. Galicki, and B. M. Mann, "The geometry and topology of 3-Sasakian manifolds," J. Reine Angew. Math. 455 (1994) 163-220.

[52] M. J. Duff, B. E. W. Nilsson, and C. N. Pope, "Kaluza-Klein supergravity," Phys. Rep. 130 (1986) 1-142.

[53] G. Tian and S.-T. Yau, "Kähler-Einstein metrics on complex surfaces with $C_{1}>0$," Comm. Math. Phys. 112 (1987) 175-203.

[54] G. Tian, "On Calabi's conjecture for complex surfaces with positive first Chern class," Invent. Math. 101 (1990) 101-172.

[55] L. J. Romans, "New compactifications of chiral $N=2, d=10$ supergravity," Phys. Lett. B 153 (1985) 392-396.

[56] L. Bérard Bergery, "Sur de nouvelles variétés riemanniennes d'Einstein," Institut Elie Cartan 6 (1982) 1-60.

[57] D. R. Morrison, "Canonical quotient singularities in dimension three," Proc. Amer. Math. Soc. 93 (1985) 393-396.

[58] R. L. Bryant and S. M. Salamon, "On the construction of some complete metrics with exceptional holonomy," Duke Math. J. 58 (1989) 829-850. 
[59] D. D. Joyce, "Compact 8-manifolds with holonomy $\operatorname{Spin}(7), "$ Invent. Math. 123 (1996) 507-552.

[60] S. Mori, D. R. Morrison, and I. Morrison, "On four-dimensional terminal quotient singularities," Math. Comp. 51 (1988) 769-786.

[61] D. R. Morrison and G. Stevens, "Terminal quotient singularities in dimensions three and four," Proc. Amer. Math. Soc. 90 (1984) 15-20.

[62] O. Aharony, Y. Oz, and Z. Yin, "M theory on $A d S_{p} \times S^{11-p}$ and superconformal field theories," Phys. Lett. B 430 (1998) 87-93, hep-th/ 9803051 .

[63] S. Sethi, "A relation between $N=8$ gauge theories in three dimensions," J. High Energy Phys. 11 (1998) 003, hep-th/9809162.

[64] L. Castellani, L. J. Romans, and N. P. Warner, "A classification of compactifying solutions for D=11 supergravity," Nucl. Phys. B 241 (1984) 429-462.

[65] M. Schlessinger, "Rigidity of quotient singularities," Invent. Math. 14 (1971) 17-26.

[66] J. Fine, "On varieties isomorphic in codimension one to torus embeddings," Duke Math. J. 58 (1989) 79-88.

[67] B. de Wit and H. Nicolai, "Extended supergravity with local $S O(5)$ invariance," Nucl. Phys. B 188 (1981) 98-108.

[68] E. Brieskorn, "Beispiele zur Differentialtopologie von Singularitäten," Invent. Math. 2 (1966) 1-14.

[69] F. Hirzebruch, "Singularities and exotic spheres," Exp. 314, Séminaire Bourbaki, vol. 1966/1967, W. A. Benjamin, New York.

[70] K. Yamagishi, "Supergravity on seven-dimensional homotopy spheres," Phys. Lett. B 134 (1984) 47-50.

[71] P. G. O. Freund, "Higher-dimensional unification," Physica D 15 (1985) 263-269.

[72] M. Kervaire and J. Milnor, "Groups of homotopy spheres, I," Ann. of Math. (2) 77 (1963) 504-537.

[73] J. Milnor, "On manifolds homeomorphic to the 7-sphere," Ann. of Math. (2) 64 (1956) 399-405.

[74] D. Gromoll and W. Meyer, "An exotic sphere with nonnegative sectional curvature," Ann. of Math. (2) 100 (1974) 401-406. 
[75] M. W. Davis, "Some group actions on homotopy spheres of dimension seven and fifteen," Amer. J. Math. 104 (1982) 59-90.

[76] P. S. Aspinwall and D. R. Morrison, "Point-like instantons on K3 orbifolds," Nucl. Phys. B 503 (1997) 533-564, hep-th/9705104.

[77] K. Intriligator, "New string theories in six dimensions via branes at orbifold singularities," Adv. Theor. Math. Phys. 1 (1997) 271-282, hep-th/9708117.

[78] E. Perevalov and G. Rajesh, "Mirror symmetry via deformation of bundles on K3 surfaces," Phys. Rev. Lett. 79 (1997) 2931-2934, hep-th/ 9706005 .

[79] K. Dasgupta and S. Mukhi, "Orbifolds of M-theory," Nucl. Phys. B 465 (1996) 399-412, hep-th/9512196.

[80] E. Witten, "Five-branes and $M$-theory on an orbifold," Nucl. Phys. B 463 (1996) 383-397, hep-th/9512219.

[81] C. Ahn, H. Kim, and H. S. Yang, " $S O(2 N)(0,2)$ SCFT and M theory on $A d S_{7} \times \mathbf{R P}^{4}$," Phys. Rev. D 59 (1999) 106002, hep-th/9808182.

[82] S. Ferrara and A. Zaffaroni, "N=1,2 4D superconformal field theories and supergravity in $A d S_{5}$," Phys. Lett. B 431 (1998) 49-56, hep-th/ 9803060 .

[83] M. Reid, "Canonical 3-folds," Journées de Géométrie Algébriqe d'Angers (A. Beauville, ed.) Sitjhoff \& Noordhoof, 1980, pp. 273-310.

[84] M. Reid, "Young person's guide to canonical singularities," Algebraic Geometry, Bowdoin, 1985, Proc. Sympos. Pure Math., vol. 46, part 1, Amer. Math. Soc., Providence, RI, 1987, pp. 345-414.

[85] P. M. H. Wilson, "The Kähler cone on Calabi-Yau threefolds," Invent. Math. 107 (1992) 561-583, Erratum, ibid. 114 (1993) 231-233.

[86] D. R. Morrison and N. Seiberg, "Extremal transitions and fivedimensional supersymmetric field theories," Nucl. Phys. B 483 (1997) 229-247, hep-th/9609070.

[87] M. R. Douglas, S. Katz, and C. Vafa, "Small instantons, del Pezzo surfaces and type I' theory," Nucl. Phys. B 497 (1997) 155-172, hep-th/ 9609071 .

[88] N. Seiberg, "Five dimensional SUSY field theories, non-trivial fixed points and string dynamics," Phys. Lett. B 388 (1996) 753-760, hep-th/9608111.

[89] A. Strominger, "Massless black holes and conifolds in string theory," Nucl. Phys. B 451 (1995) 96-108, hep-th/9504090. 
[90] B. R. Greene, D. R. Morrison, and A. Strominger, "Black hole condensation and the unification of string vacua," Nucl. Phys. B 451 (1995) 109-120, hep-th/9504145

[91] A. Klemm and P. Mayr, "Strong coupling singularities and non-abelian gauge symmetries in $N=2$ string theory," Nucl. Phys. B 469 (1996) $37-50$, hep-th/9601014.

[92] S. Katz, D. R. Morrison, and M. R. Plesser, "Enhanced gauge symmetry in type II string theory," Nucl. Phys. B 477 (1996) 105-140, hep-th/9601108.

[93] P. Candelas and X. C. de la Ossa, "Comments on conifolds," Nucl. Phys. B 342 (1990) 246-268.

[94] K. Altmann, "The versal deformation of an isolated toric Gorenstein singularity," Invent. Math. 128 (1997) 443-479, alg-geom/9403004.

[95] M. Gross, "Deforming Calabi-Yau threefolds," Math. Ann. 308 (1997) 187-220, alg-geom/9506022.

[96] P. S. Aspinwall, B. R. Greene, and D. R. Morrison, "Calabi-Yau moduli space, mirror manifolds and spacetime topology change in string theory," Nucl. Phys. B 416 (1994) 414-480, hep-th/9309097.

[97] D. R. Morrison and M. R. Plesser, "Summing the instantons: Quantum cohomology and mirror symmetry in toric varieties," Nucl. Phys. B 440 (1995) 279-354, hep-th/9412236.

[98] E. Witten, "Phases of $N=2$ theories in two dimensions," Nucl. Phys. B 403 (1993) 159-222, hep-th/9301042.

[99] P. Griffiths and J. Harris, "Principles of algebraic geometry," Wiley, 1978.

[100] R. Bott and L. W. Tu, "Differential forms in algebraic topology," Springer Verlag, New York, 1982.

[101] M. R. Douglas and G. Moore, "D-branes, quivers, and ALE instantons," hep-th/9603167.

[102] M. R. Douglas, B. R. Greene, and D. R. Morrison, "Orbifold resolution by D-branes," Nucl. Phys. B 506 (1997) 84-106, hep-th/9704151.

[103] B. R. Greene, "D-brane topology changing transitions," Nucl. Phys. B 525 (1998) 284-296, hep-th/9711124.

[104] S. Mukhopadhyay and K. Ray, "Conifolds from D-branes," Phys. Lett. B 423 (1998) 247-254, hep-th/9711131. 
[105] O. Aharony and E. Witten, "Anti-de Sitter space and the center of the gauge group," J. High Energy Phys. 11 (1998) 018, hep-th/9807205.

[106] M. Douglas, "Branes within branes," hep-th/9512077.

[107] E. Witten, "Baryons and branes in anti de Sitter space," J. High Energy Phys. 07 (1998) 006, hep-th/9805112.

[108] P. S. Aspinwall, "Enhanced gauge symmetries and K3 surfaces," Phys. Lett. B 357 (1995) 329-334, hep-th/9507012.

[109] L. I. Ibáñez, R. Rabadán, and A. M. Uranga, "Anomalous $U(1)$ 's in type I and type IIB $D=4, N=1$ string vacua," Nucl. Phys. B 542 (1999) 112-138, hep-th/9808139.

[110] M. Dine, N. Seiberg, and E. Witten, "Fayet-Iliopoulos terms in string theory," Nucl. Phys. B 289 (1987) 589-598.

[111] M. Berkooz, R. G. Leigh, J. Polchinski, J. H. Schwarz, N. Seiberg, and E. Witten, "Anomalies, dualities, and topology of $D=6 \mathrm{~N}=$ 1 superstring vacua," Nucl. Phys. B 475 (1996) 115-148, hep-th/ 9605184.

[112] A. V. Sardo Infirri, "Partial resolutions of orbifold singularities via moduli spaces of HYM-type bundles," alg-geom/9610004.

[113] J. Polchinski, "Tensors from K3 orientifolds," Phys. Rev. D 55 (1997) 6423-6428, hep-th/9606165.

[114] D. E. Diaconescu, M. R. Douglas, and J. Gomis, "Fractional branes and wrapped branes," J. High Energy Phys. 02 (1998) 013, hep-th/ 9712230.

[115] R. G. Leigh and M. J. Strassler, "Exactly marginal operators and duality in four dimensional $\mathrm{N}=1$ supersymmetric gauge theory," Nucl. Phys. B 447 (1995) 95-136, hep-th/9503121.

[116] M. A. Shifman and A. I. Vainshtein, "Solution of the anomaly puzzle in SUSY gauge theories and the Wilson operator expansion," Nucl. Phys. B 277 (1986) 456-486.

[117] M. A. Shifman and A. I. Vainshtein, "On holomorphic dependence and infrared effects in supersymmetric gauge theories," Nucl. Phys. B 359 (1991) 571-580.

[118] L. Susskind and E. Witten, "The holographic bound in anti-de Sitter space," hep-th/9805114.

[119] G. W. Gibbons and P. Rychenkova, "Cones, tri-Sasakian structures and superconformal invariance," Phys. Lett. B 443 (1998) 138-142, hep-th/9809158. 